Algebraic $\mathcal{E}$ Geometric Topology

Volume 3 (2003) 791-856

Published: 31 August 2003

ATG

\title{
Generalized orbifold Euler characteristics of symmetric orbifolds and covering spaces
}

\author{
Hirotaka TAMANOI
}

\begin{abstract}
Let $G$ be a finite group and let $M$ be a $G$-manifold. We introduce the concept of generalized orbifold invariants of $M / G$ associated to an arbitrary group $\Gamma$, an arbitrary $\Gamma$-set, and an arbitrary covering space of a connected manifold $\Sigma$ whose fundamental group is $\Gamma$. Our orbifold invariants have a natural and simple geometric origin in the context of locally constant $G$-equivariant maps from $G$-principal bundles over covering spaces of $\Sigma$ to the $G$-manifold $M$. We calculate generating functions of orbifold Euler characteristic of symmetric products of orbifolds associated to arbitrary surface groups (orientable or non-orientable, compact or non-compact), in both an exponential form and in an infinite product form. Geometrically, each factor of this infinite product corresponds to an isomorphism class of a connected covering space of a manifold $\Sigma$. The essential ingredient for the calculation is a structure theorem of the centralizer of homomorphisms into wreath products described in terms of automorphism groups of $\Gamma$-equivariant $G$-principal bundles over finite $\Gamma$-sets. As corollaries, we obtain many identities in combinatorial group theory. As a byproduct, we prove a simple formula which calculates the number of conjugacy classes of subgroups of given index in any group. Our investigation is motivated by orbifold conformal field theory.
\end{abstract}

AMS Classification 55N20, 55N91; 57S17, 57D15, 20E22, 37F20, 05A15

Keywords Automorphism group, centralizer, combinatorial group theory, covering space, equivariant principal bundle, free group, $\Gamma$-sets, generating function, Klein bottle genus, (non)orientable surface group, orbifold Euler characteristic, symmetric products, twisted sector, wreath product

\section{Introduction and summary of results}

Let $G$ be a finite group acting on a finite dimensional smooth closed manifold $M$. The action of $G$ is not assumed to be free. The orbit space $M / G$ is an example of an orbifold. In 1980s, string physicists suggested that in the context of orbifold conformal field theory the ordinary Euler characteristic $\chi(M / G)$ of 
the orbit space is not the right invariant, but the correct invariant is the orbifold Euler characteristic $e_{\mathrm{orb}}(M ; G)$ defined by

$$
e_{\text {orb }}(M ; G)=\frac{1}{|G|} \sum_{g h=h g} \chi\left(M^{\langle g, h\rangle}\right),
$$

where the summation runs over all pairs of commuting elements $(g, h)$ in $G$ [6].

We have started the investigation of generalized orbifold Euler characteristic in [17]. We continue our investigation: Our idea in this paper is to study orbifold singularities of $M / G$ through the use of a connected manifold $\Sigma$ as a "probe." More precisely, we examine an infinite dimensional mapping space $\operatorname{Map}(\Sigma, M / G)$ which can be thought of as a "thickening" of the orbit space $M / G$. We consider lifting maps $\Sigma \rightarrow M / G$ to $G$-equivariant maps $P \rightarrow M$ from a $G$-principal bundle $P$ over $\Sigma$ to $M$. The set of equivalence classes of these lifts is denoted by $\mathbb{L}_{\Sigma}(M ; G)$. We also consider a subspace of equivalence classes of locally constant $G$-equivariant maps $P \rightarrow M$ :

$$
\mathbb{L}_{\Sigma}(M ; G) \stackrel{\text { def }}{=} \coprod_{[P \longrightarrow \Sigma]} \operatorname{Map}_{G}(P, M) / \operatorname{Aut}_{G}(P) \supset \mathbb{L}_{\Sigma}(M ; G)_{\text {l.c. }}=\coprod_{[\phi] \in \operatorname{Hom}(\Gamma, G) / G}\left[M^{\langle\phi\rangle} / C(\phi)\right] .
$$

Here $[P \rightarrow \Sigma]$ runs over the set of all isomorphism classes of $G$-principal bundles over $\Sigma$ whose fundamental group is $\pi_{1}(\Sigma)=\Gamma$. Recall that isomorphism classes of $G$-bundles over $\Sigma$ are classified by the $G$-conjugacy classes $\operatorname{Hom}(\Gamma, G) / G$ of homomorphisms. For any $\phi: \Gamma \rightarrow G$, the subgroup $C(\phi) \subset G$ is the centralizer of the image of $\phi$ denoted by $\operatorname{Im} \phi=\langle\phi\rangle$, and $M^{\langle\phi\rangle}$ is the fixed point subset of $M$ under $\langle\phi\rangle$. We have $\operatorname{Aut}_{G}(P) \cong C(\phi)$. Since the space $\mathbb{L}_{\Sigma}(M ; G)$ is the space of equivalence classes of lifts, there is a natural map $\mathbb{L}_{\Sigma}(M ; G) \rightarrow \operatorname{Map}(\Sigma, M / G)$ which is a homeomorphism when $G$-action on $M$ is free. In this case, there is no need to go over the above construction, and thus we are primarily interested in non-free $G$-actions on $M$. Note that we have replaced the mapping space $\operatorname{Map}(\Sigma, M / G)$ on an orbifold $M / G$ by the space $\mathbb{L}_{\Sigma}(M ; G)$ which is a union of orbifolds on mapping spaces. As such, the space $\mathbb{L}_{\Sigma}(M ; G)$ is, in a sense, a mild desingularization of the mapping space $\operatorname{Map}(\Sigma, M / G)$. Intuitively, our manifold $\Sigma$ is used to probe the nature of orbifold singularities of the orbit space $M / G$ by examining the holonomy of all possible $G$-bundles on $\Sigma$ "induced" by a map $\gamma: \Sigma \rightarrow M / G$.

The space $\mathbb{L}_{\Sigma}(M ; G)$ is motivated by and is a generalization of the notion of twisted sectors in orbifold conformal field theory, which corresponds to the case $\Sigma=S^{1}$. Intuitively speaking, twisted sectors are vector spaces obtained by "quantizing" the mapping spaces $\operatorname{Map}_{G}(P, M)$, where $P$ runs over isomorphism classes of $G$-bundles over $S^{1}$. Thus the study of global topology and geometry 
of these equivariant mapping space will shed light on the nature of twisted sectors in orbifold conformal field theory. Mathematically, twisted sectors have been studied in the algebraic framework of vertex operator algebras and their modules. The study of geometry and topology of the space $\mathbb{L}_{\Sigma}(M ; G)$ when $\Sigma$ is $S^{1}$ or Riemann surfaces may provide illuminating topological and geometric insight into the nature of the algebraic structure of twisted sectors.

In this paper, we study the subspace of locally constant equivariant maps instead of directly studying the global topology of $\mathbb{L}_{\Sigma}(M ; G)$, and we define our generalized orbifold invariants as follows. Let $\varphi(M ; G)$ be any (multiplicative) invariant of $(M ; G)$. For example, as $\varphi(M ; G)$ we may use the following invariants coming from the usual Euler characteristic:

$$
\chi^{\text {orb }}(M ; G) \stackrel{\text { def }}{=} \frac{\chi(M)}{|G|} \in \frac{1}{|G|} \mathbb{Z}, \quad \chi(M ; G) \stackrel{\text { def }}{=} \chi(M / G) \in \mathbb{Z} .
$$

The first Euler characteristic $\chi^{\text {orb }}$ is a special case of the equivariant Euler characteristic defined for general (not necessarily finite) group $G$ [3, p.249]. The $\Gamma$-extension $\varphi_{\Gamma}(M ; G)$ of $\varphi(M ; G)$ is defined in terms of the subspace of locally constant equivariant maps given in (1-2), viewed equivariantly:

$$
\varphi_{\Gamma}(M ; G) \stackrel{\text { def }}{=} \sum_{[\phi] \in \operatorname{Hom}(\Gamma, G) / G} \varphi\left(M^{\langle\phi\rangle} ; C(\phi)\right) .
$$

This is our generalization of physicists' orbifold Euler characteristic $e_{\mathrm{orb}}(M ; G)$. In our previous paper [17], we considered $\chi_{\Gamma}(M ; G)$ for the case $\Gamma=\mathbb{Z}^{d}$ and $\Gamma=\left(\mathbb{Z}_{p}\right)^{d}$ with $d \in \mathbb{N}$, where $p$ is any prime and $\mathbb{Z}_{p}$ is the ring of $p$-adic integers. In the same paper, we described an application of the result for the case $\Gamma=\left(\mathbb{Z}_{p}\right)^{d}$ to the Euler characteristic of Morava $K$-theory of classifying spaces of wreath products. The inductive method used for these calculations cannot be extended to a general group $\Gamma$. The purpose of this paper is to develop a theory and techniques which allow us to handle orbifold invariants $\varphi_{\Gamma}(M ; G)$ for a more general family of groups $\Gamma$, including the fundamental groups of two dimensional surfaces which may be orientable or non-orientable, compact or non-compact.

For more discussion on general properties of the $\Gamma$-extension of $\varphi(M ; G)$, see section 2. For example, we will show that $\Gamma$-extensions of the two orbifold Euler characteristics in (1-3) are related by

$$
\chi_{\Gamma}(M ; G)=\chi_{\Gamma \times \mathbb{Z}}^{\mathrm{orb}}(M ; G) \in \mathbb{Z},
$$

for any group $\Gamma$. Thus, $\chi_{\Gamma \times \mathbb{Z}}^{\text {orb }}(M ; G)$ is always integer valued. When $\Gamma=\mathbb{Z}$, the invariant $\chi_{\mathbb{Z}^{2}}^{\text {orb }}(M ; G)$ coincides with the orbifold Euler characteristic (1-1) considered by physicists [6], [7]. See the formula (2-4) in section 2 . The above formula says that $\chi^{\mathrm{orb}}(M ; G)$ is more basic than $\chi(M ; G)$. 
We demonstrate that orbifold invariants (1-4) is well behaved by calculating the generating function of these invariants of symmetric products of global quotients. Recall that an $n$-fold symmetric product of an orbit space $M / G$ is given by

$$
S P^{n}(M / G)=(M / G)^{n} / \mathfrak{S}_{n}=M^{n} /\left(G \succ \mathfrak{S}_{n}\right),
$$

where $G \backslash \mathfrak{S}_{n}$, which we also denote by $G_{n}$, is the wreath product of $G$ and the symmetric group $\mathfrak{S}_{n}$. For more details on wreath products, see section 3 .

Let $\Sigma$ be a real 2 dimensional surface, orientable or non-orientable, compact or non-compact. For example, let $\Sigma$ be a genus $g+1$ orientable closed surface. Its fundamental group $\Gamma_{g+1}$ is generated by $2 g+2$ elements with one relation:

$$
\Gamma_{g+1}=\left\langle a_{1}, a_{2}, \ldots, a_{g+1}, b_{1}, b_{2}, \ldots, b_{g+1} \mid\left[a_{1}, b_{1}\right] \cdots\left[a_{g+1}, b_{g+1}\right]=1\right\rangle,
$$

where $g \geq 0$. Consideration of the twisted space (1-2) associated to the mapping space $\operatorname{Map}\left(\Sigma, S P^{n}(M / G)\right)$ from the surface $\Sigma$ to symmetric products is motivated by string theory literature [5]. We calculate the orbifold Euler characteristic $\chi_{\Gamma_{g+1}}^{\text {orb }}\left(M^{n} ; G_{n}\right)$ associated to the surface group $\Gamma_{g+1}$. It turns out that this orbifold Euler characteristic of an $n$-th symmetric product can be expressed by $\left\{\chi_{H}^{\text {orb }}(M ; G)\right\}_{H}$, where $H$ runs over subgroups of $\Gamma_{g+1}$ of index at most $n$. In fact, we have the following formula of the generating function [Theorem 5-8].

Theorem A (Higher genus orbifold Euler characteristics of symmetric orbifolds) Let $g \geq 0$. With the above notations,

$$
\sum_{n \geq 0} q^{n} \chi_{\Gamma_{g+1}}^{\mathrm{orb}}\left(M^{n} ; G \geq \mathfrak{S}_{n}\right)=\exp \left[\sum_{r \geq 1} q^{r}\left\{\frac{j_{r}\left(\Gamma_{g+1}\right)}{r} \chi_{\Gamma_{r g+1}}^{\mathrm{orb}}(M ; G)\right\}\right],
$$

where $j_{r}\left(\Gamma_{g+1}\right)$ is the number of index $r$ subgroups of $\Gamma_{g+1}$.

In this formula the genus $r g+1$ surface group $\Gamma_{r g+1}$ appears since any index $r$ subgroup of $\Gamma_{g+1}$ is isomorphic to it, although they may not be conjugate to each other in $\Gamma_{g+1}$. To calculate numbers $j_{r}\left(\Gamma_{g+1}\right)$ for $r \geq 1$ and $g \geq 0$, see the formula $(5-10)$.

Furthermore, we can also consider non-orientable cases. Let $\Sigma$ be a closed genus $h+2$ non-orientable surface with $h \geq 0$. Its fundamental group $\Lambda_{h+2}$ is described by

$$
\Lambda_{h+2}=\left\langle c_{1}, c_{2}, \ldots, c_{h+2} \mid c_{1}^{2} c_{2}^{2} \cdots c_{h+2}^{2}=1\right\rangle, \quad h \geq 0 .
$$

Since any genus 1 non-orientable closed surface is homeomorphic to $\mathbb{R} P^{2}$ and $\Lambda_{1}=\mathbb{Z} / 2 \mathbb{Z}$ is abelian, this case was discussed in our previous paper [17]. Here, 
we only consider non-orientable surfaces of genus 2 or higher. We have a formula of the generating function of the orbifold invariant $\chi_{\Lambda_{h+2}}^{\text {orb }}\left(M^{n} ; G \imath \mathfrak{S}_{n}\right)$ for nonorientable surface groups similar to the one in Theorem A. See Theorem 5-10 for details.

This formula simplifies for the case of a genus 2 non-orientable surface which is a Klein bottle, due to a fact that every finite covering of a Klein bottle is either a torus or a Klein bottle. Here we give a formula for Klein bottle orbifold Euler characteristic [Theorem 5-11].

Theorem B (Klein bottle orbifold Euler characteristic) With the above notation,

$$
\sum_{n \geq 0} q^{n} \chi_{\Lambda_{2}}^{\mathrm{orb}}\left(M^{n} ; G \backslash \mathfrak{S}_{n}\right)=\left[\prod_{r \geq 1}\left(1-q^{2 r}\right)\right]^{\frac{-1}{2} \chi_{\Gamma_{1}}^{\mathrm{orb}}(M ; G)}\left[\prod_{r \geq 1}\left(\frac{1+q^{r}}{1-q^{r}}\right)\right]^{\frac{1}{2} \chi_{\Lambda_{2}}^{\mathrm{orb}}(M ; G)} .
$$

Notice the appearance of modular forms, and modular functions. Let $\eta(q)=$ $q^{\frac{1}{24}} \prod_{r \geq 1}\left(1-q^{r}\right)$, where $q=e^{2 \pi i \tau}$ with $\operatorname{Im} \tau>0$, be the Dedekind eta function. Then, the first infinite product in (1-10) is almost $\eta\left(q^{2}\right)$ and the second infinite product is precisely $\eta\left(q^{2}\right) / \eta(q)^{2}$.

By specializing $M=\mathrm{pt}$, we obtain a formula for $\left|\operatorname{Hom}\left(\Lambda_{2}, G \imath \mathfrak{S}_{n}\right)\right|$ for all $n$. See (5-19).

Recall that the geometry behind the above Klein bottle orbifold Euler characteristic is the mapping space $\operatorname{Map}\left(K, S P^{n}(M / G)\right)$ from a Klein bottle $K$ to $n$-fold symmetric product of an orbifold $M / G$ for various $n$. The idea of using nonorientable surfaces comes from unoriented strings moving on manifolds.

There is a curious relationship between orbifold Euler characteristics associated to orientable surface groups and non-orientable surface groups. For example, when $G$ is a trivial group, we have $\chi_{\Gamma_{g+1}}^{\text {orb }}\left(M^{n} ; \mathfrak{S}_{n}\right)=\chi_{\Lambda_{2 g+2}}^{\text {orb }}\left(M^{n} ; \mathfrak{S}_{n}\right)$ for any $n \geq 1$ and $g \geq 0$. For this, see the end of section 5 .

Note that the formula in Theorem $\mathrm{A}$ is in an exponential form. If we use $\chi(M ; G)=\chi(M / G)$ instead of $\chi^{\mathrm{orb}}(M ; G)$, then the corresponding generating function can be written in an infinite product form. To describe this formula, we need to introduce an extension of $\varphi(M ; G)$ to an orbifold invariant $\varphi_{[X]}(M ; G)$ associated to an isomorphism class $[X]$ of a finite $\Gamma$-set $X$. When $X$ is a transitive $\Gamma$ set of the form $\Gamma / H$ for some subgroup $H \subset \Gamma$, we let

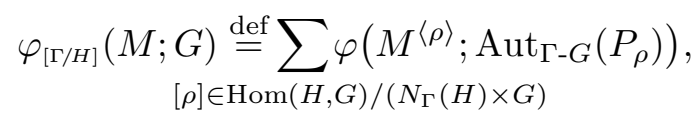


where $N_{\Gamma}(H) \subset \Gamma$ is the normalizer of $H$ in $\Gamma$ acting on $H$ by conjugation, and $\operatorname{Aut}_{\Gamma-G}\left(P_{\rho}\right)$ is the group of $\Gamma$-equivariant $G$-bundle automorphisms of a $G$ bundle $P_{\rho}=\Gamma \times{ }_{\rho} G$. The group $G$ of course acts on $\operatorname{Hom}(H, G)$ by conjugation. For an explanation and the origin of this definition, see Proposition 6-1 and its proof. The above formula reduces to (1-4) when $H=\Gamma$. For an explicit nontrivial example of $\varphi_{[\Gamma / H]}(M ; G)$, see Lemma 6-2.

The invariant $\varphi_{[X]}$ can be defined for a general $\Gamma$-set $X$ (see (6-13) for the definition) in such a way that it is multiplicative in $[X]$ in the following sense. Let $X_{1}$ and $X_{2}$ be $\Gamma$-sets without common transitive $\Gamma$-sets in their $\Gamma$-orbit decompositions, that is, $\operatorname{Map}_{\Gamma}\left(X_{1}, X_{2}\right)=\emptyset$. Then, the orbifold invariant of their disjoint union $[X]=\left[X_{1} \amalg X_{2}\right]$ is a product:

$$
\varphi_{[X]}(M ; G)=\varphi_{\left[X_{1}\right]}(M ; G) \cdot \varphi_{\left[X_{2}\right]}(M ; G) .
$$

For details, see Proposition 6-9.

This invariant $\varphi_{[X]}(M ; G)$ has a very natural geometric origin in terms of a twisted space similar to (1-2) using $G$-bundles over a covering space $\Sigma^{\prime}=$ $\widetilde{\Sigma} \times_{\Gamma} X$ over $\Sigma$, where $\widetilde{\Sigma}$ is the universal cover of $\Sigma$. See Theorem G below. In fact, we could start with this approach and deduce the formula (1-11).

The next theorem describes the generating function of the orbifold Euler characteristic of symmetric orbifolds associated to the genus $g+1$ orientable surface group $\Gamma_{g+1}$, in terms of the orbifold Euler characteristic of $(M ; G)$ associated to finite transitive $\Gamma_{g+1}$-sets.

Theorem A' (Higher genus orbifold Euler characteristic: infinite product form) For any $g \geq 0$, we have

$$
\sum_{n \geq 0} q^{n} \chi_{\Gamma_{g+1}}\left(M^{n} ; G\left\{\mathfrak{S}_{n}\right)=\prod_{[H]}\left(1-q^{\left|\Gamma_{g+1} / H\right|}\right)^{-\chi_{\left[\Gamma_{g+1} / H\right]}(M ; G)},\right.
$$

where $[H]$ runs overthe set of conjugacy classes of $H$ in $\Gamma_{g+1}$.

This is part of Theorem 6-6. As remarked above, the invariant $\chi_{\left[\Gamma_{g+1} / H\right]}(M ; G)$ with $\left|\Gamma_{g+1} / H\right|=r$ arises from a twisted space (1-21) defined in terms of $G$ principal bundles over an $r$-fold covering space $\Sigma_{r g+1}$ over $\Sigma_{g+1}$ corresponding to the conjugacy class $[H]$.

To contrast the formulae in Theorem A and Theorem $\mathrm{A}^{\prime}$, we let $M=$ pt. We get the following combinatorial group theoretic formulae:

$$
\begin{aligned}
\sum_{n \geq 0} q^{n} \frac{\left|\operatorname{Hom}\left(\Gamma_{g+1}, G_{n}\right)\right|}{|G|^{n} \cdot n !} & =\exp \left[\sum_{r \geq 1} \frac{q^{r}}{r} j_{r}\left(\Gamma_{g+1}\right) \frac{\left|\operatorname{Hom}\left(\Gamma_{r g+1}, G\right)\right|}{|G|}\right] \\
\sum_{n \geq 0} q^{n}\left|\operatorname{Hom}\left(\Gamma_{g+1}, G_{n}\right) / G_{n}\right| & =\prod_{[H]}\left(1-q^{\left|\Gamma_{g+1} / H\right|}\right)^{-\left|\operatorname{Hom}(H, G) /\left(N_{\Gamma_{g+1}}(H) \times G\right)\right|}
\end{aligned}
$$


In the second formula, $[H]$ runs over all conjugacy classes of finite index subgroups of $\Gamma_{g+1}$. Note that the size of the normalizer $N_{\Gamma_{g+1}}(H)$ may depend on the conjugacy class $[H]$, although all index $r$ subgroups of $\Gamma_{g+1}$ are isomorphic to $\Gamma_{r g+1}$. See also remarks after Theorem 6-6.

Here we remark that the following formulae for the number of (conjugacy classes of) homomorphisms are well known, and they can be easily proved using characters of $G$. For any finite group $G$, we have

$$
\begin{aligned}
\frac{\left|\operatorname{Hom}\left(\Gamma_{g+1}, G\right)\right|}{|G|} & =\sum_{[V] \in \operatorname{Irred}(G)}\left(\frac{|G|}{\operatorname{dim} V}\right)^{2 g}, \\
\left|\operatorname{Hom}\left(\Gamma_{g+1}, G\right) / G\right| & =\sum_{[\sigma]} \sum_{[V] \in \operatorname{Irred}(C(\sigma))}\left(\frac{|C(\sigma)|}{\operatorname{dim} V}\right)^{2 g},
\end{aligned}
$$

where $[V] \in \operatorname{Irred}(G)$ means that $[V]$ runs over the set of all isomorphism classes of irreducible representations of $G$, and $[\sigma]$ runs over the set of all conjugacy classes of $G$. The subgroup $C(\sigma) \subset G$ is the centralizer of $\sigma$. Similar formulae for non-orientable surface groups $\Lambda_{h+2}$ are well known, too. See (5-16) and (5-17). In view of (1-15), to understand the left hand side of (1-14), we need the representation theory of wreath product $G_{n}=G \mathfrak{S}_{n}$. For this topic, see for example [11, Appendix B to Chapter I]. However, to prove topological Theorems $\mathrm{A}$ and $\mathrm{A}^{\prime}$, we do not need the representation theory of wreath products.

Two formulae in (1-14) are closely related, although their right hand sides look very different. See (6-11) and (6-12) for group theoretic discussion on this connection.

Theorems $\mathrm{A}, \mathrm{B}$, and $\mathrm{A}^{\prime}$ are all special cases of the following more general formulae which are the ones we actually prove [Theorem 5-5, Theorem 6-3]. These are part of our main results.

Theorem C With the above notations, for any group $\Gamma$, we have

$$
\begin{aligned}
\sum_{n \geq 0} q^{n} \chi_{\Gamma}^{\mathrm{orb}}\left(M^{n} ; G \imath \mathfrak{S}_{n}\right) & =\exp \left[\sum_{H} \frac{q^{|\Gamma / H|}}{|\Gamma / H|} \chi_{H}^{\mathrm{orb}}(M ; G)\right] \\
\sum_{n \geq 0} q^{n} \chi_{\Gamma}\left(M^{n} ; G \imath \mathfrak{S}_{n}\right) & =\prod_{[H]}\left(1-q^{|\Gamma / H|}\right)^{-\chi_{[\Gamma / H]}(M ; G)} .
\end{aligned}
$$

Here, in the first formula, $H$ runs over all subgroups of $\Gamma$ of finite index. In the second formula, $[H]$ runs over conjugacy classes of all finite index subgroups of $\Gamma$. 
By specializing $\Gamma$, we obtain numerous corollaries. In addition to the cases $\Gamma=\Gamma_{g+1}, \Lambda_{h+2}$, we consider free groups on finitely many generators, abelian groups $\mathbb{Z}^{d}$ and $\mathbb{Z}_{p}^{d}$ for $d \geq 1$, and products of these groups.

Special cases of the second formula in (1-16) have been known. When $\Gamma$ is the trivial group, this is Macdonald's formula [10]. When $\Gamma=\mathbb{Z}$ and $G=\{e\}$, this formula is due to [7]. For $\Gamma=\mathbb{Z}$ and general finite group $G$, the formula is due to [18]. When $\Gamma=\mathbb{Z}^{d}$ for $d \geq 1$ and $G=\{e\}$, the formula was proved in [4]. Finally, for $\Gamma=\mathbb{Z}^{d}$ and $\mathbb{Z}_{p}^{d}$ with an arbitrary finite group $G$, the formula was proved in [17].

When $G$ is the trivial group, we have $\chi_{[X]}(M ;\{e\})=\chi(M)$ for any transitive $\Gamma$-set $X$ (see Lemma 6-10, and a formula after Corollary 6-4). When $\Gamma$ is an abelian group $\mathbb{Z}^{d}$ or $\mathbb{Z}_{p}^{d}$, Lemma 6 -2 says that $\chi_{[X]}(M ; G)=\chi_{\Gamma}(M ; G)$ for any finite transitive $\Gamma$-set $X$. For general abelian group $\Gamma$, the second formula in (1-16) reduces to (6-5). Thus, in all the known cases described in the above paragraph, the notion of orbifold invariants associated to $\Gamma$-sets is not yet needed. Only in the generality of the present paper, this notion plays a crucial role.

The main ingredient of the proof of Theorem $\mathrm{C}$ is a structure theorem of the centralizer $C(\theta) \subset G_{n}$ of an arbitrary homomorphism $\theta: \Gamma \rightarrow G_{n}=G \imath \mathfrak{S}_{n}$ into a wreath product. When $\Gamma=\mathbb{Z}$, the subgroup $C(\theta)$ is nothing but the centralizer of the element $\theta(1) \in G_{n}$. Detailed description of the centralizer of an element in $G_{n}$ was given in section 3 of [17]. The method used there was purely group theoretic. It is very complicated to extend this group theoretic approach to the present context of centralizers of homomorphisms into wreath products. We have a better approach in terms of geometry of $\Gamma$-equivariant $G$-principal bundles. These are $G$-principal bundles $P$ over $\Gamma$-sets such that $\Gamma$ acts on $P$ as $G$-bundle automorphisms. This link between algebra and geometry is supplied by the following theorem [Theorem 3-1]. For the next three theorems, $G$ does not have to be a finite group.

Theorem $\mathbf{D}$ Let $G$ and $\Gamma$ be any group. Then the following bijective correspondence exists:

$$
\left\{\begin{array}{l}
\text { Isomorphism classes of } \Gamma \text {-equivariant } G \text {-principal } \\
\text { bundles over a } \Gamma \text {-set of order } n
\end{array}\right\} \underset{\text { onto }}{\stackrel{1: 1}{\longrightarrow}} \operatorname{Hom}\left(\Gamma, G_{n}\right) / G_{n} \text {. }
$$

This theorem allows us to apply geometric concepts and techniques associated to principal bundles to the study of centralizers of homomorphisms $\theta$ into wreath products. Note that this theorem is a generalization of a well-known 
bijective correspondence between the set $\operatorname{Hom}\left(\Gamma, \mathfrak{S}_{n}\right) / \mathfrak{S}_{n}$ of conjugacy classes of homomorphisms into the $n$-th symmetric group $\mathfrak{S}_{n}$ and the set of isomorphism classes of $\Gamma$-sets of order $n$. As an example of the above correspondence, let $H \subset \Gamma$ be any subgroup of index $n$. For any homomorphism $\rho: H \rightarrow G$, let $P_{\rho}=\Gamma \times{ }_{\rho} G \rightarrow \Gamma / H$ be a $\Gamma$-equivariant $G$-principal bundle over a $\Gamma$-set $\Gamma / H$ of order $n$. By choosing a bijection $\Gamma / H \cong\{1,2, \ldots, n\}$ and a section of $P_{\rho} \rightarrow \Gamma / H$, we have an isomorphism $\operatorname{Aut}_{G}\left(P_{\rho}\right) \cong G \mathfrak{S}_{n}$ [Lemma 3-3]. Thus, the wreath product appears naturally in the geometric context of $G$-bundles. This is the reason of our use of $G$-bundles in studying wreath products. Now the ready-made action of $\Gamma$ on $P_{\rho}$ as $G$-bundle maps gives rise to a homomorphism $\theta: \Gamma \rightarrow G \imath \mathfrak{S}_{n}$ whose conjugacy class is independent of choices made. For more details on this and the construction of the converse correspondence, see the proof of Theorem 3-1.

We call a $\Gamma$-equivariant $G$-principal bundle over a $\Gamma$-transitive set a $\Gamma$-irreducible $G$-principal bundle. The following theorem classifies $\Gamma$-irreducible $G$ principal bundles [Theorem 3-6].

Theorem E (Classification of $\Gamma-G$ bundles) Let $G$ and $\Gamma$ be any groups. Then there exists the following bijective correspondence:

(1-18)

$\left\{\begin{array}{l}\text { Isomorphism classes of } \Gamma \text {-irreducible } G \text { - } \\ \text { principal bundles over } \Gamma \text {-sets of order } n\end{array}\right\} \underset{\text { onto }}{\stackrel{1: 1}{[H]}} \coprod_{|\Gamma / H|=n} \operatorname{Hom}(H, G) /\left(N_{\Gamma}(H) \times G\right)$,

where $[H]$ runs over the set of conjugacy classes of index $n$ subgroups of $\Gamma$.

Note that the expression appearing on the right hand side of the above correspondence has already appeared in (1-11). From the point of view of a $\Gamma$ irreducible $G$-principal bundle $P \rightarrow Z$, the meaning of quantities $[H], N_{\Gamma}(H)$ action, and $G$-action on $\operatorname{Hom}(H, G)$, appearing in the right hand side of (1-18), is as follows: the isomorphism class of the transitive $\Gamma$-set $Z$ is determined by the conjugacy class $[H]$ of an isotropy subgroup $H$, different choices of a base point $z_{0}$ of $Z$ with the isotropy subgroup $H$ correspond to the action of $N_{\Gamma}(H)$, and different choices of a base point $p_{0}$ of $P$ over $z_{0}$ correspond to the conjugation action of $G$ on $\operatorname{Hom}(H, G)$.

For any homomorphism $\theta: \Gamma \rightarrow G_{n}$, the corresponding $\Gamma$-equivariant $G$ bundle $\pi_{\theta}: P_{\theta} \rightarrow Z_{\theta}$ is a disjoint union of $\Gamma$-irreducible $G$-bundles each of which is of the form $P_{\rho} \rightarrow \Gamma / H$ for some subgroup $H$ and some homomorphism $\rho: H \rightarrow G$. Given $\theta$, let $r(H, \rho)$ denote the number of $\Gamma$-irreducible $G$ bundles isomorphic to $\left[P_{\rho} \rightarrow \Gamma / H\right]$ appearing in the irreducible decomposition 
of $P_{\theta} \rightarrow Z_{\theta}$. The number $r(H, \rho)$ depends only on the conjugacy classes $[H]$ and $[\rho]$. Now we can describe the centralizer of the image of $\theta$ in the wreath product $G \imath \mathfrak{S}_{n}$ [Lemma 4-1, Theorem 4-2, Theorem 4-4, Theorem 4-5].

Theorem F (Structure of centralizer of homomorphisms into wreath products) Let $\theta: \Gamma \rightarrow G_{n}$ be a homomorphism, and let $\{r(H, \rho)\}_{[H],[\rho]}$ be the associated integers described above. Then, the centralizer of $\theta$ in the wreath product $G \geq \mathfrak{S}_{n}$ is a direct product of wreath products:

$$
C_{G_{n}}(\theta) \cong \prod_{[H]} \prod_{[\rho]}\left\{\operatorname{Aut}_{\Gamma-G}\left(P_{\rho}\right) \imath \mathfrak{S}_{r(H, \rho)}\right\},
$$

where $[H]$ runs over conjugacy classes of finite index subgroups, and $[\rho] \in$ $\operatorname{Hom}(H, G) /\left(N_{\Gamma}(H) \times G\right)$ for a given conjugacy class $[H]$. The group $\operatorname{Aut}_{\Gamma-G}\left(P_{\rho}\right)$ of $\Gamma$-equivariant $G$-bundle automorphisms of $P_{\rho}$ fits into the following exact sequences:

$$
\begin{gathered}
1 \rightarrow C_{G}(\rho) \rightarrow \operatorname{Aut}_{\Gamma-G}\left(P_{\rho}\right) \rightarrow H \backslash N_{\Gamma}^{\rho}(H) \rightarrow 1, \\
1 \rightarrow H \rightarrow T_{\rho} \rightarrow \operatorname{Aut}_{\Gamma-G}\left(P_{\rho}\right) \rightarrow 1,
\end{gathered}
$$

where $N_{\Gamma}^{\rho}(H)$ is the isotropy subgroup at the $G$-conjugacy class $(\rho)$ of the $N_{\Gamma}(H)$-action on the set $\operatorname{Hom}(H, G) / G$, and $T_{\rho}$ is given in (4-4).

Here, the centralizer $C_{G_{n}}(\theta)$ is isomorphic to $\operatorname{Aut}_{\Gamma-G}\left(P_{\theta}\right)$ [Lemma 4-1].

This structure theorem can be applied to much wider context including, for example, the calculation of generalized orbifold elliptic genus of symmetric orbifolds.

Next, we describe the geometric meaning of the orbifold invariant (1-11) associated to $\Gamma$-sets. Recall that the orbifold invariant associated to the group $\Gamma$ is defined in terms of locally constant $G$-equivariant maps (1-2) from $G$ principal bundles over $\Sigma$ into $M$, where the fundamental group of $\Sigma$ is $\Gamma$. We can generalize this idea and consider locally constant $G$-equivariant maps from $G$-principal bundles over covering spaces of $\Sigma$ into $M$. More precisely, let $\pi: \Sigma^{\prime} \rightarrow \Sigma$ be a not necessarily connected covering space. Let $\pi_{i}: P_{i} \rightarrow \Sigma^{\prime}$ for $i=1,2$ be $G$-bundles. A $G$-bundle isomorphism $\alpha: P_{1} \rightarrow P_{2}$ over a covering space $\Sigma^{\prime}$ are defined to be a $G$-bundle map whose induced map on the base $\Sigma^{\prime}$ is a deck transformation of $\Sigma^{\prime}$ over $\Sigma$. Next, we introduce an equivalence relation among $G$-maps $P \rightarrow M$. Two $G$-equivariant maps $\gamma_{1}: P_{1} \rightarrow M$ and $\gamma_{2}: P_{2} \rightarrow M$ are said to be equivalent if there exists a $G$-bundle isomorphism $\alpha: P_{1} \rightarrow P_{2}$ over the covering space $\Sigma^{\prime}$ such that $\gamma_{1}=\gamma_{2} \circ \alpha$. We then consider 
the space of equivalence classes of $G$-equivariant maps from $G$-bundles $P$ over $\Sigma^{\prime}$ into $M$. This turns out to be

$$
\mathbb{L}_{\Sigma^{\prime} / \Sigma}(M ; G)=\coprod_{\left[P \rightarrow \Sigma^{\prime} / \Sigma\right]}\left[\operatorname{Map}_{G}(P, M) / \operatorname{Aut}_{G}(P)_{\Sigma^{\prime} / \Sigma}\right],
$$

where $[P \rightarrow \Sigma / \Sigma]$ denotes an isomorphism classes of $G$-principal bundles over the covering space $\Sigma^{\prime}$ over $\Sigma$. We then take the subset $\mathbb{L}_{\Sigma^{\prime} / \Sigma}(M ; G)_{\text {l.c. }}$ of locally constant $G$-equivariant maps and define the orbifold invariant associated to a covering space $\Sigma^{\prime} \rightarrow \Sigma$ by

$$
\varphi_{\left[\Sigma^{\prime} \Sigma\right]}(M ; G)=\sum_{\left[P \longrightarrow \Sigma^{\prime} / \Sigma\right]} \varphi\left(\operatorname{Map}_{G}(P, M)_{\text {l.c. }} ; \operatorname{Aut}_{G}(P)_{\Sigma^{\prime} / \Sigma}\right) .
$$

The next result [Theorem 7-1] clarifies the geometric meaning of orbifold Euler characteristic associated to $\Gamma$-sets. See (6-13) for the precise definition.

Theorem G Let $\pi: \Sigma^{\prime} \rightarrow \Sigma$ be a covering space, and let $X=\pi^{-1}\left(x_{0}\right)$ be a fibre over a base point $x_{0} \in \Sigma$. Let $\Gamma=\pi_{1}\left(\Sigma, x_{0}\right)$. Then $X$ is a $\Gamma$-set and for any $G$-manifold $M$, we have

$$
\varphi_{\left[\Sigma^{\prime} / \Sigma\right]}(M ; G)=\varphi_{[X]}(M ; G) .
$$

Consequently, $\Gamma$-orbifold Euler characteristic of symmetric products of $G$ manifold $M$ can be expressed in terms of orbifold Euler characteristic associated to connected covering spaces:

$$
\sum_{n \geq 0} q^{n} \chi_{\Gamma}\left(M^{n} ; G \geq \mathfrak{S}_{n}\right)=\prod_{\left[\Sigma^{\prime} \longrightarrow \Sigma\right]_{\mathrm{conn}} .}\left(1-q^{\left|\Sigma^{\prime} / \Sigma\right|}\right)^{-\chi_{\left[\Sigma^{\prime} / \Sigma\right]}(M ; G)},
$$

where the product range over all isomorphism classes of finite connected covering spaces $\Sigma^{\prime} \rightarrow \Sigma$ over $\Sigma$, and $\left|\Sigma^{\prime} / \Sigma\right|$ denotes the order of the covering.

Note that the definition (1-22) is geometrically very natural and conceptually very simple, compared with the practical definition (1-11) of orbifold invariants associated to transitive $\Gamma$-sets which is suitable for calculation.

The above formula is very interesting because it explains the geometric origin of the infinite product (one factor for each connected covering space) and describes each factor geometrically in terms of covering spaces.

Intuitive explanation of this identity may be given as follows. There are two ways to lift maps in $\operatorname{Map}\left(\Sigma, S P^{n}(M / G)\right)$ to equivariant maps from certain principal bundles. One way is to lift a map $\bar{\gamma}: \Sigma \rightarrow S P^{n}(M / G)$ to a $G$ < $\mathfrak{S}_{n^{-}}$

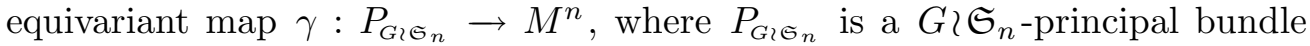


over $\Sigma$. The orbifold invariant using this lifting gives rise to the left hand side of $(1-24)$.

To describe another way of lifting, note that any map $f: \Sigma \rightarrow S P^{n}(M)$ into an $n$-th symmetric product corresponds to a (branched) $n$-sheeted covering space $\pi: \Sigma^{\prime} \rightarrow \Sigma$ whose fibre over $x \in \Sigma$ is $f(x) \subset M$. That is, $\Sigma^{\prime}$ is given by $\Sigma^{\prime}=\{(x, y) \in \Sigma \times M \mid y \in f(x)\}$. This is not always a covering space because for some $x \in \Sigma$ the corresponding un-ordered set of $n$ points $f(x)$ may not consist of $n$ distinct points. The (branched) covering space $\Sigma^{\prime}$ comes equipped with a natural map $f^{\prime}: \Sigma^{\prime} \rightarrow M$ which is unique up to "deck transformations" of $\Sigma^{\prime}$. Namely, if we have a homeomorphism $h: \Sigma^{\prime} \rightarrow \Sigma^{\prime}$ commuting with $\pi$, then both maps $f^{\prime}$ and $f^{\prime} \circ h$ induce the same map $f: \Sigma \rightarrow S P^{n}(M)$ by associating to each $x \in \Sigma$ the fibres of $f^{\prime}$ and $f^{\prime} \circ h$.

Now in our context, to any map $\bar{\gamma}: \Sigma \rightarrow S P^{n}(M / G)$ we cab associate a map $\bar{\gamma}^{\prime}: \Sigma^{\prime} \rightarrow M / G$ from an $n$-sheeted (branched) covering space $\Sigma^{\prime}$ over $\Sigma$ described above. This map $\bar{\gamma}^{\prime}$ is determined up to "deck transformations" of $\Sigma^{\prime}$. We then consider lifting this map to a $G$-equivariant map $\gamma^{\prime}: P_{G} \rightarrow M$, where $P_{G}$ is a $G$-principal bundle over $\Sigma^{\prime}$. Orbifold invariants corresponding to these lifts are the orbifold invariants associated to covering spaces in the right hand side of (1-24). We expect the covering space formalism in (1-24) would be valid for other orbifold invariants and also for the global topology of twisted sectors.

We suspect that the formula (1-24) will continue to be valid for general orbifolds which are not necessarily global quotients. We hope to come back to this question later.

Finally, as an application to combinatorial group theory, we calculate the number $u_{r}(\Gamma)$ of conjugacy classes of index $r$ subgroups of $\Gamma$. Our combinatorial formulae obtained by specializing our topological formulae to the case $M=\mathrm{pt}$ allow us to prove the following formula on $u_{r}(\Gamma)$ [Theorem 8-1]. This simple formula does not seem to be known before.

Theorem $\mathbf{H}$ The number $u_{r}(\Gamma)$ of conjugacy classes of index $r$ subgroups of $\Gamma$ satisfies the following recursive relation in terms of subgroups of $\Gamma$ :

$$
j_{m}(\Gamma \times \mathbb{Z})=\sum_{r \mid m} r \cdot u_{r}(\Gamma)=\sum_{r \mid m} \sum_{\substack{H \\|\Gamma / H|=m / r}}\left|\operatorname{Hom}\left(H_{\mathrm{ab}}, \mathbb{Z}_{r}\right)\right|,
$$

where $H_{\mathrm{ab}}$ denotes the abelianization of $H$. 
If we like, instead of having a recursive formula as above, we can write down the formula for $u_{r}(\Gamma)$ using Möbius inversion formula. See the paragraph after Theorem 8-1. The above formula allows us to compute numbers $u_{r}(\Gamma)$ for surface groups $\Gamma_{g+1}, F_{s+1}$, and $\Lambda_{h+2}$ for $g, s, h \geq 0$ very quickly [Corollary 8-2]. Geometrically these numbers $u_{r}(\Gamma)$ are important because they count the number of non-isomorphic connected covering spaces over surfaces. These numbers were calculated before in [12], [13] by different methods. However, our formula (1-25) is valid for any group $\Gamma$, not just for surface groups. In our formula, all we have to do is to count the number of homomorphisms from abelianization of subgroups to cyclic groups. This simplicity makes our formula useful.

The organization of this paper is as follows. In section 2, we discuss general properties of $\Gamma$-extended orbifold invariants and explain their geometric origin. In section 3 , we study homomorphisms into wreath products in terms of $\Gamma$ equivariant $G$-bundles and prove Theorems D and E. In section 4, we study the structure of centralizers of homomorphisms into wreath products in terms of $\Gamma$-equivariant $G$-automorphisms and prove Theorem F. In section 5 , we prove Theorems A and B, together with the first part of Theorem C. We also discuss the cases when $\Gamma$ is a free abelian group, the fundamental group of a nonorientable surface, and a free group. In section 6 , we further extend orbifold invariants to ones associated to isomorphism classes of $\Gamma$-sets. This will be used to prove Theorem $\mathrm{A}^{\prime}$ and Theorem C. Our main results in this paper deal with much more general cases than [17], and results in [17] follow quickly as special cases. In section 7 , we describe orbifold invariants associated to covering spaces and prove Theorem G. Finally, in section 8, we apply our combinatorial formula to compute the numbers $u_{r}(\Gamma)$ and prove Theorem $\mathrm{H}$.

\section{Orbifold invariants associated to a group $\Gamma$}

In this section, we describe general properties of the $\Gamma$-extended orbifold invariant $\varphi_{\Gamma}(M ; G)$ defined in (1-4). In particular, properties of the $\Gamma$-extensions of orbifold Euler characteristics defined in (1-3) are discussed in detail. Later in this section, we explain twisted spaces in the context of mapping spaces into orbifolds and motivate the definition of our generalized orbifold invariant (1-4).

Let $G$ be a finite group and let $\varphi(M ; G)$ be a multiplicative invariant for a $G$-manifold $M$. Namely, for any $G_{1}$-manifold $M_{1}$ and $G_{2}$-manifold $M_{2}$, the invariant $\varphi$ satisfies

$$
\varphi\left(M_{1} \times M_{2} ; G_{1} \times G_{2}\right)=\varphi\left(M_{1} ; G_{1}\right) \varphi\left(M_{2} ; G_{2}\right) .
$$


This invariant $\varphi(M ; G)$ may depend only on the topology and geometry of the orbit space $M / G$. For example, Euler characteristic $\chi(M / G)$, signature $\operatorname{Sgn}(M / G)$, and spin index $\operatorname{Spin}(M / G)$, in appropriate geometric settings, are such examples. On the other hand, the invariant $\varphi(M ; G)$ may actually depend on the way $G$ acts on $M$. For example, for any group $\Gamma, \Gamma$-extensions of the above geometric invariants are such examples. For convenience, we repeat the definition of $\Gamma$-extension here. For any such invariant $\varphi(\cdot ; \cdot)$, its $\Gamma$-extension is defined by

$$
\varphi_{\Gamma}(M ; G)=\sum_{[\phi] \in \operatorname{Hom}(\Gamma, G) / G} \varphi\left(M^{\langle\phi\rangle} ; C(\phi)\right),
$$

where $M^{\langle\phi\rangle}$ is the $\langle\phi\rangle=\operatorname{Im} \phi$ fixed point subset, and $C(\phi)$ is the centralizer of $\langle\phi\rangle$ in $G$. Note that it can happen that two non-conjugate homomorphisms $\phi_{1}, \phi_{2}: \Gamma \rightarrow G$ have the same image subgroups $\left\langle\phi_{1}\right\rangle=\left\langle\phi_{2}\right\rangle$. Also note that the quantity $\varphi\left(M^{\langle\phi\rangle} ; C(\phi)\right)$ depends only on the $G$-conjugacy class of $\phi$.

Basic properties of $\Gamma$-extended orbifold invariants are the following ones.

Proposition 2-1 Let $\varphi(M ; G)$ be a multiplicative invariant as above.

(1) For any group $\Gamma$, the $\Gamma$-extended orbifold invariant $\varphi_{\Gamma}(M ; G)$ is multiplicative: for any $\left(M_{1} ; G_{1}\right)$ and $\left(M_{2} ; G_{2}\right)$, we have

$$
\varphi_{\Gamma}\left(M_{1} \times M_{2} ; G_{1} \times G_{2}\right)=\varphi_{\Gamma}\left(M_{1} ; G_{1}\right) \varphi_{\Gamma}\left(M_{2} ; G_{2}\right) .
$$

(2) For any groups $K, L$,

$$
\varphi_{K \times L}(M ; G)=\left(\varphi_{L}\right)_{K}(M ; G)=\sum_{[\phi] \in \operatorname{Hom}(K, G) / G} \varphi_{L}\left(M^{\langle\phi\rangle} ; C(\phi)\right) .
$$

Proof The formula in (1) is straightforward using the multiplicativity of $\varphi$. For (2), by definition,

$$
\text { (R.H.S.) }=\sum_{\phi_{1}: K \rightarrow G} \frac{1}{\#\left[\phi_{1}\right]}\left\{\sum_{\phi_{2}: L \rightarrow C\left(\phi_{1}\right)} \frac{1}{\#\left[\phi_{2}\right]_{C\left(\phi_{1}\right)}} \varphi\left(\left(M^{\left\langle\phi_{1}\right\rangle}\right)^{\left\langle\phi_{2}\right\rangle} ; C_{C\left(\phi_{1}\right)}\left(\phi_{2}\right)\right)\right\}
$$

Here, $\#\left[\phi_{1}\right]$ denotes the number of elements in the conjugacy class $\left[\phi_{1}\right]$, and $\left[\phi_{2}\right]_{C\left(\phi_{1}\right)}$ is the $C\left(\phi_{1}\right)$-conjugacy class of $\phi_{2}$. Since the images of $\phi_{1}$ and $\phi_{2}$ commute, using the notation $\phi_{1} \times \phi_{2}: K \times L \rightarrow G$, we have

$$
\begin{aligned}
& =\sum_{\phi_{1}: K \rightarrow G} \frac{1}{\#\left[\phi_{1}\right]} \sum_{\phi_{2}: L \rightarrow C\left(\phi_{1}\right)} \frac{\left|C\left(\phi_{1} \times \phi_{2}\right)\right|}{\left|C\left(\phi_{1}\right)\right|} \varphi\left(M^{\left\langle\phi_{1} \times \phi_{2}\right\rangle} ; C\left(\phi_{1} \times \phi_{2}\right)\right) \\
& =\sum_{\phi_{1} \times \phi_{2}: K \times L \rightarrow G} \frac{\left|C\left(\phi_{1} \times \phi_{2}\right)\right|}{|G|} \varphi\left(M^{\left\langle\phi_{1} \times \phi_{2}\right\rangle} ; C\left(\phi_{1} \times \phi_{2}\right)\right) \\
& =\sum_{\left[\phi_{1} \times \phi_{2}\right]} \varphi\left(M^{\left\langle\phi_{1} \times \phi_{2}\right\rangle} ; C\left(\phi_{1} \times \phi_{2}\right)\right)=\varphi_{K \times L}(M ; G) .
\end{aligned}
$$

Algebraic \&S Geometric Topology, Volume 3 (2003) 
In the last line, $\left[\phi_{1} \times \phi_{2}\right]$ runs over all the conjugacy classes $\operatorname{Hom}(K \times L, G) / G$, and we used a fact that the number of elements in the conjugacy class of the image of $\phi_{1} \times \phi_{2}$ is $|G| /\left|C\left(\phi_{1} \times \phi_{2}\right)\right|$. In the above, we used a formula $|G|=$ $\#[\phi] \cdot|C(\phi)|$ a few times. This completes the proof.

Recall that in section 1, we defined two kinds of Euler characteristics for $(M ; G)$ in (1-3). One of them is $\chi^{\mathrm{orb}}(M ; G)=\chi(M) /|G|$, and the other is $\chi(M ; G)=$ $\chi(M / G)$. It is well known that the Euler characteristic of the orbit space $M / G$ of a $G$-manifold $M$ can be calculated as the average of the Euler characteristic of $g$-fixed point subsets (see for example [14, p.127]):

$$
\chi(M / G)=\frac{1}{|G|} \sum_{g \in G} \chi\left(M^{\langle g\rangle}\right) .
$$

Here are some useful formulae for $\Gamma$-extended orbifold Euler characteristics.

Proposition 2-2 (1) The invariant $\chi_{\Gamma}^{\mathrm{orb}}(M ; G)$ can be calculated as an average over homomorphisms $\phi: \Gamma \rightarrow G$ :

$$
\chi_{\Gamma}^{\mathrm{orb}}(M ; G)=\frac{1}{|G|} \sum_{\phi: \Gamma \rightarrow G} \chi\left(M^{\langle\phi\rangle}\right) .
$$

In particular, $\chi_{\mathbb{Z}}^{\mathrm{orb}}(M ; G)=\chi(M / G)$.

(2) The $\Gamma$-extensions of $\chi^{\text {orb }}(M ; G)$ and $\chi(M ; G)$ are related as follows:

$$
\chi_{\Gamma \times \mathbb{Z}}^{\text {orb }}(M ; G)=\chi_{\Gamma}(M ; G)=\sum_{[\phi] \in \operatorname{Hom}(\Gamma, G) / G} \chi\left(M^{\langle\phi\rangle} / C(\phi)\right) .
$$

Proof For part (1), we unravel definitions.

$$
\chi_{\Gamma}^{\mathrm{orb}}(M ; G)=\sum_{[\phi]} \chi^{\mathrm{orb}}\left(M^{\langle\phi\rangle} ; C(\phi)\right)=\sum_{\phi} \frac{1}{\#[\phi]} \frac{\chi\left(M^{\langle\phi\rangle}\right)}{|C(\phi)|}=\frac{1}{|G|} \sum_{\phi} \chi\left(M^{\langle\phi\rangle}\right),
$$

where $[\phi] \in \operatorname{Hom}(\Gamma, G) / G$ and $\phi \in \operatorname{Hom}(\Gamma, G)$. This proves formula (2-4), In particular, when $\Gamma=\mathbb{Z}$, we have $\chi_{\mathbb{Z}}^{\text {orb }}(M ; G)=(1 /|G|) \sum_{g \in G} \chi\left(M^{\langle g\rangle}\right)$, which is equal to $\chi(M / G)$ by $(2-3)$.

For (2), by the product formula (2-2), we have

$$
\chi_{\Gamma \times \mathbb{Z}}^{\mathrm{orb}}(M ; G)=\sum_{[\phi] \in \operatorname{Hom}(\Gamma, G) / G} \chi_{\mathbb{Z}}^{\mathrm{orb}}\left(M^{\langle\phi\rangle} ; C(\phi)\right)=\sum_{[\phi]} \chi\left(M^{\langle\phi\rangle} / C(\phi)\right)=\chi_{\Gamma}(M ; G) .
$$

This completes the proof. 
For example, when $\Gamma=\mathbb{Z}^{2}$, we have

$$
\chi_{\mathbb{Z}^{2}}^{\mathrm{orb}}(M ; G)=\frac{1}{|G|} \sum_{g h=h g} \chi\left(M^{\langle g, h\rangle}\right)=\sum_{[g]} \chi\left(M^{\langle g\rangle} / C(g)\right),
$$

which is the physicist's orbifold Euler characteristic (1-1). The following corollary is obvious.

Corollary 2-3 When $M=\mathrm{pt}$, we have

$$
\chi_{\Gamma}^{\text {orb }}(\mathrm{pt} ; G)=\frac{|\operatorname{Hom}(\Gamma, G)|}{|G|}, \quad \chi_{\Gamma}(\mathrm{pt} ; G)=|\operatorname{Hom}(\Gamma, G) / G|=\frac{|\operatorname{Hom}(\Gamma \times \mathbb{Z}, G)|}{|G|} .
$$

When $G$ is the trivial group $\{e\}$, we have

$$
\chi_{\Gamma}^{\mathrm{orb}}(M ;\{e\})=\chi_{\Gamma}(M ;\{e\})=\chi(M) .
$$

Here, the identity $|\operatorname{Hom}(\Gamma, G) / G|=|\operatorname{Hom}(\Gamma \times \mathbb{Z}, G)| /|G|$ comes from $\chi_{\Gamma}(\mathrm{pt} ; G)$ $=\chi_{\Gamma \times \mathbb{Z}}^{\text {orb }}(\mathrm{pt} ; G)$ given in $(2-5)$. This is a well-known and useful formula.

When $\chi_{\Gamma}^{\text {orb }}(\cdot ; G)$ is regarded as a function on $G$-CW complexes with values in $\frac{1}{|G|} \mathbb{Z}$, it is an additive function. If either $G$ or $\Gamma$ is abelian, it is a complex oriented additive function in the sense of [8]. Since an additive function defined on $G$-CW complexes is completely determined by its values on transitive $G$ sets, we calculate these values. We also do the same for $\chi_{\Gamma}(\cdot ; G)$.

Proposition 2-4 Let $H$ be a subgroup of $G$. Then

$$
\begin{aligned}
\chi_{\Gamma}^{\mathrm{orb}}(G / H ; G) & =\chi_{\Gamma}^{\mathrm{orb}}(\mathrm{pt} ; H)=\frac{|\operatorname{Hom}(\Gamma, H)|}{|H|} \\
\chi_{\Gamma}(G / H ; G) & =\chi_{\Gamma}(\mathrm{pt} ; H)=|\operatorname{Hom}(\Gamma, H) / H| .
\end{aligned}
$$

Proof Using (2-4), we have

$$
\chi_{\Gamma}^{\mathrm{orb}}(G / H ; G)=\frac{1}{|G|} \sum_{\phi: \Gamma \longrightarrow G} \chi\left((G / H)^{\langle\phi\rangle}\right)=\frac{1}{|G|} \sum_{\phi}\left|(G / H)^{\langle\phi\rangle}\right| .
$$

Here $\left|(G / H)^{\langle\phi\rangle}\right|$ counts the number of elements in $G / H$ whose isotropy subgroups contain $\phi(\Gamma)$. Since there are $\left|N_{G}(H) / H\right|$ elements in $G / H$ with the same isotropy subgroups, we have

$$
\left|(G / H)^{\langle\phi\rangle}\right|=\sum_{\substack{\phi(\Gamma) \subset H^{\prime} \\ H^{\prime} \sim H}}\left|N_{G}(H) / H\right|
$$


Here $H^{\prime} \sim H$ means that $H^{\prime}$ and $H$ are conjugate in $G$. Continuing our calculation, we have

$$
\begin{aligned}
\frac{1}{|G|} \sum_{\phi}\left|(G / H)^{\langle\phi\rangle}\right| & =\frac{\left|N_{G}(H) / H\right|}{|G|} \sum_{\substack{\phi \\
\phi(\Gamma) \subset H^{\prime} \\
H^{\prime} \sim H}} 1=\frac{\left|N_{G}(H)\right|}{|G||H|} \sum_{H^{\prime} \sim H} \sum_{\phi: \Gamma \rightarrow H^{\prime}} 1 \\
& =\frac{\left|N_{G}(H)\right|}{|G||H|}|\operatorname{Hom}(\Gamma, H)| \sum_{H^{\prime} \sim H} 1 .
\end{aligned}
$$

since the number of subgroups $H^{\prime}$ conjugate to $H$ in $G$ is given by $|G| /\left|N_{G}(H)\right|$, the above quantity is equal to $|\operatorname{Hom}(\Gamma, H)| /|H|$.

For $\chi_{\Gamma}(\cdot ; G)$, using $(2-5),(2-6)$, and what we have already proved, we have

$$
\chi_{\Gamma}(G / H ; G)=\chi_{\Gamma \times \mathbb{Z}}^{\mathrm{orb}}(G / H ; G)=\chi_{\Gamma \times \mathbb{Z}}^{\mathrm{orb}}(\mathrm{pt} ; H)=\chi_{\Gamma}(\mathrm{pt} ; H)=|\operatorname{Hom}(\Gamma, H) / H| .
$$

This completes the proof.

Next, we turn our attention to orbifold Euler characteristics of symmetric products without $\Gamma$-extension. Macdonald's formula [10] says that

$$
\sum_{n \geq 0} q^{n} \chi\left(M^{n} ; G 乙 \mathfrak{S}_{n}\right)=\sum_{n \geq 0} q^{n} \chi\left(S P^{n}(M / G)\right)=\frac{1}{(1-q)^{\chi(M / G)}} .
$$

The corresponding formula for $\chi^{\mathrm{orb}}(\cdot ; \cdot)$ is given by

$$
\sum_{n \geq 0} q^{n} \chi^{\mathrm{orb}}\left(M^{n} ; G \imath \mathfrak{S}_{n}\right)=\exp \left\{q \frac{\chi(M)}{|G|}\right\}=\exp \left\{q \chi^{\mathrm{orb}}(M ; G)\right\} .
$$

This formula follows immediately since $\chi^{\text {orb }}\left(M_{n} ; G \mathfrak{S}_{n}\right)=\chi(M)^{n} /\left(|G|^{n} n !\right)$ by definition. The objective of this paper is to develop a theory and a technique to calculate orbifold invariants with $\Gamma$-extensions of symmetric products.

For the remaining part of this section, we explain the notion of twisted sectors (or spaces) from which follows the geometric origin of our definition of the orbifold invariant (2-1) associated to a group $\Gamma$. The notion of twisted sectors arises in orbifold conformal field theory [6] in which strings moving in an orbifold $M / G$ are considered. This situation is analyzed through their lifts to $M$. When lifted to $M$, a string may not close, but its end points are related by the action of an element $g \in G$. Thus, we are led to consider the $g$-twisted free loop space $L_{g} M$ for $g \in G$ given by

$$
L_{g} M=\{\gamma: \mathbb{R} \rightarrow M \mid \gamma(t+1)=g \cdot \gamma(t) \text { for all } t \in \mathbb{R}\} .
$$

On this space the centralizer $C(g)$ acts. The $g$-twisted sector in orbifold conformal field theory is, intuitively, a vector space obtained by "quantizing" $L_{g} M$ 
(read: by taking a certain function space on $L_{g} M$ ), and as such $C(g)$ acts projectively on this vector space.

The same point of view can be applied to more general context. Let $\Sigma$ be any connected manifold. We consider $\Sigma$ moving in an orbifold $M / G$. The basic configuration space is a mapping space $\operatorname{Map}(\Sigma, M / G)$. To analyze this situation, we consider their lifts to $G$-equivariant maps from $G$-principal bundles $P$ over $\Sigma$ to $M$. Since the action of $G$ on $M$ is not assumed to be free, not all maps $\bar{\gamma}: \Sigma \rightarrow M / G$ lift to $G$-equivariant maps from principal bundles. Even when they do, there can be lifts from several non-isomorphic principal bundles to $M$. We consider the set of all possible lifts $\gamma$ of $\bar{\gamma} \in \operatorname{Map}(\Sigma, M / G)$ for all possible $G$-principal bundles $P$ over $\Sigma$, and we introduce an equivalence relation among lifts: two lifts $\gamma_{1}: P_{1} \rightarrow M$ and $\gamma_{2}: P_{2} \rightarrow M$ of $\bar{\gamma}$ are equivalent if there exists a $G$-bundle isomorphism $\alpha: P_{1} \stackrel{\cong}{\rightrightarrows} P_{2}$ inducing the identity map on $\Sigma$ such that $\gamma_{2} \circ \alpha=\gamma_{1}$. We denote the set of all equivalence classes by $\mathbb{L}_{\Sigma}(M ; G)$. There is a natural map from $\mathbb{L}_{\Sigma}(M ; G)$ to the original mapping space. Thus we have

$$
\pi_{\Sigma}: \mathbb{L}_{\Sigma}(M ; G)=\coprod_{[P]} \operatorname{Map}_{G}(P, M) / \operatorname{Aut}_{G}(P) \rightarrow \operatorname{Map}(\Sigma, M / G) .
$$

Here the union runs over all the isomorphism classes of $G$-principal bundles over $\Sigma$. When the $G$-action on $M$ is free, the above map $\pi_{\Sigma}$ is a homeomorphism. In general, the map $\pi_{\Sigma}$ is a kind of mild resolution of orbifold singularities. Note that $\mathbb{L}_{\Sigma}(M ; G)$ is still an orbifold, although the group $\operatorname{Aut}_{G}(P)$ is in general a proper subgroup of $G$ for any $P$. The part of $\mathbb{L}_{\Sigma}(M ; G)$ corresponding to

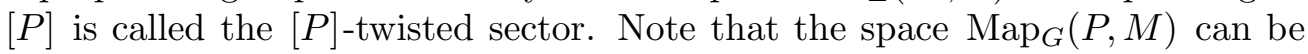
described as the space of sections of a fibre bundle $P_{M}=P \times_{G} M \rightarrow \Sigma$.

The same space can be described in a different way. Since the isomorphism class of a $G$-principal bundle $P \rightarrow \Sigma$ is determined by the $G$-conjugacy class of an associated holonomy homomorphism $\phi: \pi_{1}(\Sigma) \rightarrow G$ and since the group of $G$-bundle automorphisms $\operatorname{Aut}_{G}(P)$ of a $G$-bundle $P \rightarrow \Sigma$ associated to $\phi$ is given by the centralizer $C(\phi) \subset G$, the space of total twisted sectors $\mathbb{L}_{\Sigma}(M ; G)$ can be described alternately as follows:

$$
\mathbb{L}_{\Sigma}(M ; G)=\coprod_{[\phi]} \operatorname{Map}_{\phi}(\widetilde{\Sigma}, M) / C(\phi) .
$$

Here the disjoint union runs over all $G$-conjugacy classes of homomorphisms $\phi: \pi_{1}(\Sigma) \rightarrow G$, the space $\widetilde{\Sigma}$ is the universal cover of $\Sigma$, and the $\phi$-twisted mapping space $\operatorname{Map}_{\phi}$ is defined by

$$
\operatorname{Map}_{\phi}(\widetilde{\Sigma}, M)=\left\{\gamma: \widetilde{\Sigma} \rightarrow M \mid \gamma(p h)=\phi(h)^{-1} \gamma(p) \text { for all } p \in \widetilde{\Sigma}, h \in \pi_{1}(\Sigma)\right\} \text {. }
$$


We call $\operatorname{Map}_{\phi}(\widetilde{\Sigma}, M)$ the $\phi$-twisted sector.

Our future goal is to investigate global topology of the infinite dimensional twisted space $\mathbb{L}_{\Sigma}(M ; G)$. In this paper, we examine the subspace of $(2-11)$ consisting of locally constant $G$-equivariant maps. The same space can be described as the space of constant $\phi$-equivariant maps in (2-12). Although this is a very small portion of the space $\mathbb{L}_{\Sigma}(M ; G)$, it already contains very interesting information. This space of (locally) constant equivariant maps comes equipped with a map to $M / G$ :

$$
\bar{\pi}_{\Sigma}: \coprod_{[\phi]} M^{\langle\phi\rangle} / C(\phi) \rightarrow M / G,
$$

where $[\phi] \in \operatorname{Hom}(\Gamma, G) / G$, with $\Gamma=\pi_{1}(\Sigma)$. Note that the space corresponding to the trivial $\phi$ is $M / G$, and the map $M^{\langle\phi\rangle} / C(\phi) \rightarrow M / G$ is the obvious map: mapping $C(\phi)$-orbits to $G$-orbits in $M$. By considering the Euler characteristic of the left hand side, we get our definition (2-1) of the orbifold Euler characteristic associated to the group $\Gamma$. In this form, the group $\Gamma$ can be any group, it does not have to be the fundamental group of a manifold. This gives our definition (2-1) for any group $\Gamma$. However, interesting cases are for those groups $\Gamma$ arising as fundamental groups of manifolds.

\section{Geometry of homomorphisms into wreath prod- ucts}

For results in this section and the next, the group $G$ does not have to be finite. We assume finiteness only in the orbifold context.

Let $M$ be a $G$-manifold. On the $n$-fold Cartesian product $M^{n}$, the direct product $G^{n}$ acts coordinate-wise, and also the $n$-th symmetric group $\mathfrak{S}_{n}$ acts by permuting factors. Combining these two actions, we have an action of the wreath product $G_{n}=G \backslash \mathfrak{S}_{n}$ on $M^{n}$, which is formally defined as follows. Let $\mathbf{n}=\{1,2, \ldots, n\}$ for $n \geq 1$. The $n$-th symmetric group $\mathfrak{S}_{n}$ is, by definition, the totality of bijections of $\mathbf{n}$ to itself. The wreath product $G \mathfrak{S}_{n}$ is defined as a semi-direct product

$$
G \imath \mathfrak{S}_{n}=\operatorname{Map}(\mathbf{n}, G) \rtimes \mathfrak{S}_{n},
$$

where $\sigma \in \mathfrak{S}_{n}$ acts on $f \in \operatorname{Map}(\mathbf{n}, G)$ by $(\sigma \cdot f)(k)=f\left(\sigma^{-1}(k)\right)$ for $k \in \mathbf{n}$. The product and inverse in $G 2 \mathfrak{S}_{n}$ is given by $\left(f_{1}, \sigma_{1}\right)\left(f_{2}, \sigma_{2}\right)=\left(f_{1} \cdot \sigma_{1} f_{2}, \sigma_{1} \sigma_{2}\right)$ and $(f, \sigma)^{-1}=\left(\sigma^{-1} f^{-1}, \sigma^{-1}\right)$ for any $f_{1}, f_{2}, f \in \operatorname{Map}(\mathbf{n}, G)$ and $\sigma_{1}, \sigma_{2}, \sigma \in \mathfrak{S}_{n}$. When $n=0$, we set $G_{n}$ to be the trivial group. 
The left action of $(f, \sigma) \in G \imath \mathfrak{S}_{n}$ on $\left(x_{1}, x_{2}, \ldots, x_{n}\right) \in M^{n}$ is given by (3-2)

$$
(f, \sigma)\left(x_{1}, x_{2}, \ldots, x_{n}\right)=\left(f(1) x_{\sigma^{-1}(1)}, f(2) x_{\sigma^{-1}(2)}, \ldots, f(n) x_{\sigma^{-1}(n)}\right) \in M^{n} .
$$

In the calculation of the $\Gamma$-extended orbifold invariant $\varphi_{\Gamma}\left(M^{n} ; G \succ \mathfrak{S}_{n}\right)$ of the $n$-th symmetric product of an orbifold (see (5-1) for an explicit expression), we have to deal with homomorphisms into wreath products. Let $\theta: \Gamma \rightarrow G i \mathfrak{S}_{n}$ be such a homomorphism. Let $\theta(u)=(f(u, \cdot), \phi(u))$, where $f(u, \cdot) \in$ $\operatorname{Map}(\mathbf{n}, G)$, and $\phi: \Gamma \rightarrow \mathfrak{S}_{n}$ is the composition of $\theta$ and the canonical projection map $G \geq \mathfrak{S}_{n} \rightarrow \mathfrak{S}_{n}$. Through $\phi$, any $\theta$ induces a $\Gamma$-set structure on $\mathbf{n}$. That $\theta$ is a homomorphism immediately implies that the two variable function $f(\cdot, \cdot): \Gamma \times \mathbf{n} \rightarrow G$ satisfies the following identities:

$$
\begin{aligned}
f\left(u_{1} u_{2}, \ell\right) & =f\left(u_{1}, \ell\right) f\left(u_{2}, \phi\left(u_{1}\right)^{-1}(\ell)\right) \\
f(1, \ell) & =1 \\
f(u, \ell)^{-1} & =f\left(u^{-1}, \phi(u)^{-1}(\ell)\right)
\end{aligned}
$$

for any $u_{1}, u_{2} \in \Gamma$, and $\ell \in \mathbf{n}$.

Recall that there is a bijective correspondence between the conjugacy classes of homomorphisms $\operatorname{Hom}\left(\Gamma, \mathfrak{S}_{n}\right) / \mathfrak{S}_{n}$ and the set of isomorphism classes of $\Gamma$-sets of order $n$. We prove a similar correspondence for homomorphisms into wreath products. Namely,

Theorem 3-1 Let $G$ and $\Gamma$ be any group. Then the following bijective correspondence exists:

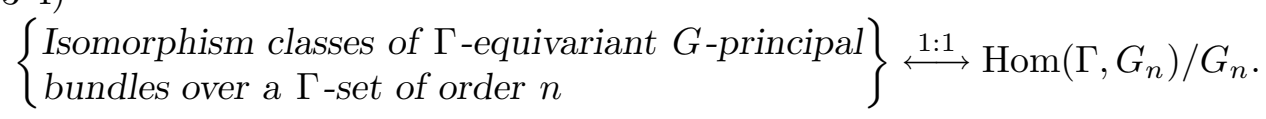

Before proving this theorem, we discuss some generalities on $\Gamma$-equivariant $G$ principal bundles. These objects arise naturally in the context of $G$-bundles over finite covering spaces. See section 7 for details. Let $\pi: P \rightarrow Z$ be such a bundle. Then $P \rightarrow Z$ is a (right) $G$-principal bundle over a left $\Gamma$-set $Z$ and $\Gamma$ acts $G$-equivariantly on $P$ from the left, inducing the $\Gamma$-action on $Z$. Thus, the left $\Gamma$ action and the right $G$ action on $P$ commute. We can also let $\Gamma \times G$ act on $P$ from the right by setting $p \cdot(u, g)=u^{-1} p g \in P$ for any $p \in P, u \in \Gamma$ and $g \in G$. We use both of these two view points on group actions on $P$.

For $p \in P$, let $x=\pi(p) \in Z$ and let $H_{x}$ be the isotropy subgroup at $x \in Z$ of the $\Gamma$ action on $Z$. Since any $h \in H_{x}$ preserves the fibre $\pi^{-1}(x)$, for any point $p \in \pi^{-1}(x)$ we may write $h \cdot p=p \cdot \rho_{p}(h)$ for some unique $\rho_{p}(h) \in G$. Since left $\Gamma$ action and the right $G$ action commute, it can be easily checked 
that $\rho_{p}: H_{x} \rightarrow G$ is a homomorphism. Thus, to each point $p \in P$, there is an associated homomorphism $\rho_{p}$. At any two points $p$ and $p^{\prime}$ of $P$ over the same $\Gamma$-orbit in $Z$, we can easily check that the corresponding homomorphisms $\rho_{p}$ and $\rho_{p^{\prime}}$ are related by

$$
\rho_{p^{\prime}}\left(u h u^{-1}\right)=g^{-1} \rho_{p}(h) g, \quad p^{\prime}=u p g,
$$

for some $u \in \Gamma$ and $g \in G$. Here $h \in H_{\pi(p)}$ so that $u h u^{-1} \in H_{\pi\left(p^{\prime}\right)}$.

The basic structure of a $\Gamma$-equivariant $G$-principal bundle over a $\Gamma$-transitive set is simple.

Lemma 3-2 Let $\pi: P \rightarrow Z$ be a $\Gamma$-equivariant $G$-principal bundle over a transitive $\Gamma$-set $Z$. For any point $p_{0} \in P$, let $\rho: H \rightarrow G$ be the homomorphism associated to $p_{0} \in P$, where $H \subset \Gamma$ is the isotropy subgroup at $\pi(p) \in Z$ so that $Z \cong \Gamma / H$ as $\Gamma$-sets. Then, we have a $\Gamma$-equivariant $G$-principal bundle isomorphism $\Gamma \times{ }_{\rho} G \cong P$, where $[u, g] \in \Gamma \times{ }_{\rho} G$ corresponds to up $p_{0} g \in P$. Here, the equivalence relation in $P_{\rho}=\Gamma \times{ }_{\rho} G$ is given by $[u, g]=\left[u h, \rho(h)^{-1} g\right]$ for all $h \in H$.

The proof is straightforward. One notes that $\Gamma \times G$ acts transitively on $P$ from the right whose isotropy subgroup at $p_{0} \in P$ is $\{(h, \rho(h)) \mid h \in H\} \cong H$.

The reason of our use of $G$-bundles in our study of wreath products is that wreath products arise naturally as the full automorphisms groups of $G$-bundles over finite sets.

Lemma 3-3 The group of $G$-bundle automorphisms of the trivial $G$-bundle over the set $\mathbf{n}$ is the wreath product $G \imath \mathfrak{S}_{n}$. That is, $\operatorname{Aut}_{G}(\mathbf{n} \times G)=G \imath \mathfrak{S}_{n}$. Here, the action of $(f, \sigma) \in G \backslash \mathfrak{S}_{n}$ given in (3-1) on $(\ell, g) \in \mathbf{n} \times G$ is given by $(f, \sigma) \cdot(\ell, g)=(\sigma(\ell), f(\sigma(\ell)) g)$.

One can easily check that this defines an action. This action is compatible with the action (3-2) in the following way. Through the identification $M^{n} \cong$ $\operatorname{Map}_{G}(\mathbf{n} \times G, M)$ given by the evaluation of $G$-equivariant maps at $1 \in G$, the action of $(f, \sigma)$ on $M^{n}$ given in (3-2) corresponds exactly to the action $\left((f, \sigma)^{-1}\right)^{*}$ on $\operatorname{Map}_{G}(\mathbf{n} \times G, M)$.

Lemma 3-3 gives a geometric characterization of wreath products. This is the very reason why $G$-principal bundles are relevant to our study of wreath products.

We are ready to prove Theorem 3-1. 
Proof of Theorem 3-1 First we describe the correspondence (3-4) and show that it is well defined.

For a given $\Gamma$-equivariant $G$-principal bundle $\pi: P \rightarrow Z$ with $|Z|=n$, we choose a section $s: Z \rightarrow P$ and a bijection $t: \mathbf{n} \stackrel{\cong}{\longrightarrow} Z$. We then have a $G$-bundle isomorphism $\mathbf{n} \times G \underset{t}{\stackrel{\cong}{\longrightarrow}} Z \times G \underset{s}{\stackrel{\cong}{\longrightarrow}} P$. We transfer the $\Gamma$ action on $P$ to an action on $\mathbf{n} \times G$ through this isomorphism. Since the action of $\gamma \in \Gamma$ on $P$ is $G$-equivariant, the corresponding action $\theta(\gamma)$ on $\mathbf{n} \times G$ is also $G$-equivariant. Since $\operatorname{Aut}_{G}(\mathbf{n} \times G)=G \imath \mathfrak{S}_{n}$ by Lemma 3-3, we have a homomorphism $\theta: \Gamma \rightarrow G\left\{\mathfrak{S}_{n}\right.$.

We show that the conjugacy class $[\theta]$ is independent of the choices $(s, t)$ and depends only on the isomorphism class of $\pi: P \rightarrow Z$ as a $\Gamma$-equivariant $G$ bundle. Let $\pi_{1}: P_{1} \rightarrow Z_{1}$ and $\pi_{2}: P_{2} \rightarrow Z_{2}$ be two isomorphic $\Gamma$-equivariant $G$-bundles. Let $\varphi: P_{1} \stackrel{\cong}{\longrightarrow} P_{2}$ be an isomorphism. Let $\left(s_{1}, t_{1}\right)$ and $\left(s_{2}, t_{2}\right)$ be the choices as above for $P_{1}$ and $P_{2}$. We have the following commutative diagram.

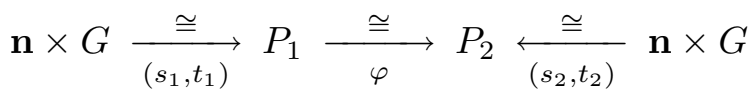

$$
\begin{aligned}
& \cong \downarrow \theta_{1}(\gamma) \quad \cong \downarrow \gamma \quad \cong \downarrow \gamma \quad \cong \downarrow \theta_{2}(\gamma)
\end{aligned}
$$

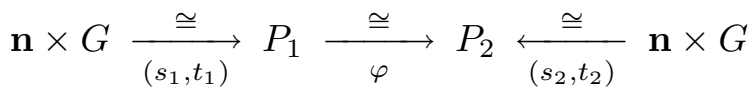

The composition of horizontal maps is a $G$-bundle isomorphism $\eta$ of $\mathbf{n} \times G$, and hence $\eta \in G \mathfrak{S}_{n}$, and the commutativity of the diagram shows that $\theta_{2}(\gamma)=$ $\eta \theta_{1}(\gamma) \eta^{-1}$. Thus, $\theta_{1}, \theta_{2}: \Gamma \rightarrow G \geq \mathfrak{S}_{n}$ are conjugate in $G \geq \mathfrak{S}_{n}$. This proves that the correspondence (3-4) is well defined.

Next we show that the correspondence (3-4) is onto. Let $\theta: \Gamma \rightarrow G$ ᄀ $\mathfrak{S}_{n}$ be a homomorphism. Let $P_{\theta}$ be $\mathbf{n} \times G$ with $\Gamma$-action induced by $\theta: \Gamma \rightarrow$ $\operatorname{Aut}_{G}\left(P_{\theta}\right)=G \imath \mathfrak{S}_{n}$. Since the left $\Gamma$-action commutes with the right $G$-action on $P_{\theta}$, this $\Gamma$-action induces a $\Gamma$-action on $\mathbf{n}$. Hence $P_{\theta} \rightarrow \mathbf{n}$ is a $\Gamma$-equivariant $G$-bundle. With the obvious choice of $(s, t)$, the homomorphism corresponding to $P_{\theta} \rightarrow \mathbf{n}$ is clearly $\theta: \Gamma \rightarrow G \backslash \mathfrak{S}_{n}$. Hence the correspondence (3-4) is onto.

We give another proof of surjectivity. In this proof, a bundle $P \rightarrow Z$ corresponding to $\theta$ is constructed from a different point of view as a union of $\Gamma$-irreducible $G$-bundles. This description clarifies the detailed internal structures of the bundle $P \rightarrow Z$, and it will be useful for later sections. Let $\theta: \Gamma \rightarrow G<\mathfrak{S}_{n}$ be a homomorphism. Let $\phi: \Gamma \stackrel{\theta}{\rightarrow} G<\mathfrak{S}_{n} \stackrel{\text { proj }}{\longrightarrow} \mathfrak{S}_{n}$ be a composition. Let $\theta(u)=(f(u, \cdot), \phi(u))$ for $u \in \Gamma$. Let $\mathbf{n}=\coprod_{\xi} X_{\xi}$ be the orbit decomposition of $\mathbf{n}$ under the $\Gamma$-action on $\mathbf{n}$ defined by $\phi$. We 
choose a base point $x_{\xi} \in X_{\xi}$ from each orbit, and let $H_{\xi}$ be the isotropy subgroup at $x_{\xi}$. Now $\theta$ decomposes as $\theta=\prod_{\xi} \theta_{\xi}: \Gamma \rightarrow \prod_{\xi} G \imath \mathfrak{S}\left(X_{\xi}\right)$, where $\theta_{\xi}: \Gamma \rightarrow G \backslash \mathfrak{S}\left(X_{\xi}\right)$, and $\mathfrak{S}\left(X_{\xi}\right)$ is the group of permutations of $X_{\xi}$. For each $\xi$, let $\rho_{\xi}: H_{\xi} \rightarrow G$ be defined by $\rho_{\xi}(u)=f\left(u, x_{\xi}\right)$ for $u \in H_{\xi}$. Since $H_{\xi}$ fixes $x_{\xi}$, the first formula in (3-3) shows that $\rho_{\xi}$ is a homomorphism. Now set $P=\coprod_{\xi}\left(\Gamma \times \rho_{\xi} G \rightarrow X_{\xi}\right)$. This $P$ is a $\Gamma$-equivariant $G$-principal bundle over $\mathbf{n}$. We show that by choosing sections for these bundles appropriately, the homomorphism corresponding to $P$ is precisely $\theta$ we started with. Now a section $s_{\xi}$ for $P_{\xi}=\Gamma \times_{\rho_{\xi}} G \rightarrow X_{\xi}$ is the same as a $\rho_{\xi}$-equivariant map $s_{\xi}: \Gamma \rightarrow G$. Let $s_{\xi}(u)=f\left(u^{-1}, x_{\xi}\right)$ for $u \in \Gamma$. By (3-3), for $h \in H_{\xi}$ we have $s_{\xi}(u h)=f\left(h^{-1} u^{-1}, x_{\xi}\right)=f\left(h^{-1}, x_{\xi}\right) f\left(u^{-1}, \phi(h) x_{\xi}\right)=\rho_{\xi}(h)^{-1} s_{\xi}(u)$, where $\phi(h) x_{\xi}=x_{\xi}$ since $h \in H_{\xi}$. This shows that $s_{\xi}$ is $\rho_{\xi}$-equivariant. Thus, a map $\alpha_{\xi}: X_{\xi} \rightarrow \Gamma \times_{\rho_{\xi}} G$ given by $\alpha_{\xi}\left(\phi(u) x_{\xi}\right)=\left[u, s_{\xi}(u)\right]$ for $u \in \Gamma$ is a well defined section of $P_{\xi} \rightarrow X_{\xi}$. Using this section, we have a $G$-bundle isomorphism $X_{\xi} \times G \rightarrow \Gamma \times \times_{\rho_{\xi}} G$ mapping $\left(\phi(u) x_{\xi}, g\right)$ to $\alpha_{\xi}\left(\phi(u) x_{\xi}\right) g=\left[u, s_{\xi}(u) g\right]$. We transfer the $\Gamma$ action on $\Gamma \times{ }_{\rho_{\xi}} G$ to $X_{\xi} \times G$ using this isomorphism. Let $\theta_{\xi}(\gamma)$ be the action on $X_{\xi} \times G$ corresponding to the action of $\gamma$ on $\Gamma \times \rho_{\rho_{\xi}} G$, in which the action of $\Gamma$ is given by $\gamma\left[u, s_{\xi}(u) g\right]=\left[\gamma u, s_{\xi}(u) g\right]$. Let $\theta_{\xi}(\gamma)\left(\phi(u) x_{\xi}, g\right)=\left(\phi(\gamma u) x_{\xi}, g^{\prime}\right)$ for some $g^{\prime} \in G$. The corresponding element in $\Gamma \times_{\rho_{\xi}} G$ is $\left[\gamma u, s_{\xi}(\gamma u) g^{\prime}\right]$ which must be equal to $\left[\gamma u, s_{\xi}(u) g\right]$. Using (3-3), we have $s_{\xi}(\gamma u)=s_{\xi}(u) f\left(\gamma, \phi(\gamma u) x_{\xi}\right)^{-1}$, hence $g^{\prime}=f\left(\gamma, \phi(\gamma u) x_{\xi}\right) g$. This means that $\theta_{\xi}(\gamma)\left(\phi(u) x_{\xi}, g\right)=\left(\phi(\gamma u) x_{\xi}, f\left(\gamma, \phi(\gamma u) x_{\xi}\right) g\right)=(f(\gamma),, \phi(\gamma)) \cdot\left(\phi(u) x_{\xi}, g\right)$ by the description of the action of $G_{n}$ on $\mathbf{n} \times G$ given in Lemma 3-3. This shows that the $\Gamma$-action $\theta_{\xi}^{\prime}$ on $X_{\xi} \times G$ is given by $\theta_{\xi}^{\prime}(\gamma)=(f(\gamma),, \phi(\gamma))$, which is the original $\theta$ restricted to the bundle over $X_{\xi}$, namely $\theta_{\xi}: \Gamma \rightarrow G \imath \mathfrak{S}\left(X_{\xi}\right)$. Hence the homomorphism $\prod \theta_{\xi}^{\prime}$ corresponding to the $\Gamma$-equivariant $G$-bundle $P=\coprod_{\xi}\left(\Gamma \times \rho_{\xi} G \rightarrow X_{\xi}\right)$ with chosen sections $\left\{\alpha_{\xi}\right\}_{\xi}$ is precisely $\theta: \Gamma \rightarrow G \mathfrak{S}_{n}$. This proves that the correspondence (3-4) is onto.

Finally, we show that the correspondence (3-4) is injective. Let $\pi_{i}: P_{i} \rightarrow Z_{i}$ for $i=1,2$ be two $\Gamma$-equivariant $G$-bundles such that corresponding homomorphisms $\theta_{1}$ and $\theta_{2}$, after making some choices $\left(s_{i}, t_{i}\right)$ for $i=1,2$, are conjugate by an element $\eta \in G \geq \mathfrak{S}_{n}$. We then have the following commutative diagram.

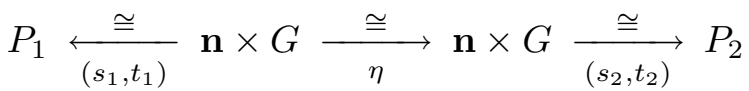

$$
\begin{aligned}
& \cong \downarrow \gamma \quad \cong \downarrow \theta_{1}(\gamma) \quad \cong \downarrow \theta_{2}(\gamma) \quad \cong \downarrow \gamma
\end{aligned}
$$

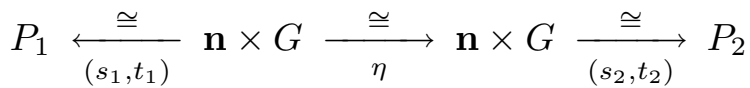

The composition $\varphi$ of the horizontal maps is a $G$-bundle isomorphism. The commutativity of the diagram shows that $\varphi: P_{1} \rightarrow P_{2}$ is a $G$-bundle map 
commuting with the action of $\Gamma$. Hence $\varphi$ is a $\Gamma$-equivariant $G$-bundle isomorphism. This shows that the correspondence in (3-4) is injective. This completes the proof.

Next we discuss isomorphisms between $\Gamma$-equivariant $G$-principal bundles. Recall that for any point $p \in P$, we can associate a homomorphism $\rho_{p}: H_{\pi(p)} \rightarrow$ $G$. This homomorphism completely characterize the $\Gamma$ - $G$ bundle $P \rightarrow Z$ when the base is a transitive $\Gamma$-set.

Proposition 3-4 (1) Let $\pi_{i}: P_{i} \rightarrow Z_{i}$ be $\Gamma$-equivariant $G$-bundles for $i=1,2$. Let $\varphi: P_{1} \cong P_{2}$ be $\Gamma$-equivariant $G$-bundle isomorphism. Then for every $p_{1} \in P_{1}$, letting $p_{2}=\varphi\left(p_{1}\right)$, we have

$$
\rho_{p_{1}}=\rho_{p_{2}}: H \rightarrow G,
$$

where $H=H_{\pi_{1}\left(p_{1}\right)}=H_{\pi_{2}\left(p_{2}\right)} \subset \Gamma$ is the isotropy subgroup at $\pi_{1}\left(p_{1}\right) \in Z_{1}$ and $\pi_{2}\left(p_{2}\right) \in Z_{2}$.

(2) Conversely, if there exists $p_{1} \in P_{1}$ and $p_{2} \in P_{2}$ such that $\rho_{p_{1}}=\rho_{p_{2}}$ and if $Z_{1}$ and $Z_{2}$ are transitive $\Gamma$-sets, then there exists a unique $\Gamma$-equivariant $G$-bundle isomorphism $\varphi: P_{1} \stackrel{\cong}{\leftrightarrows} P_{2}$ such that $\varphi\left(p_{1}\right)=p_{2}$.

Proof The $\Gamma$-equivariant $G$-bundle isomorphism $\varphi$ induces a $\Gamma$-isomorphism $\bar{\varphi}: Z_{1} \rightarrow Z_{2}$ on the base sets. Hence isotropy subgroups at $x_{1}=\pi_{1}\left(p_{1}\right)$ and $x_{2}=\pi_{2}\left(p_{2}\right)=\bar{\varphi}\left(x_{1}\right)$ must be the same subgroup of $\Gamma$ which we call $H$. Let the homomorphisms associated to points $p_{1}$ and $p_{2}$ be $\rho_{1}, \rho_{2}: H \rightarrow G$. For any $h \in H, \Gamma$-equivariance of $\varphi$ and the definition of $\rho_{2}$ imply that $\varphi\left(h p_{1}\right)=$ $h \varphi\left(p_{1}\right)=h p_{2}=p_{2} \rho_{2}(h)$. On the other hand, $G$-equivariance implies that $\varphi\left(h p_{1}\right)=\varphi\left(p_{1} \rho_{1}(h)\right)=\varphi\left(p_{1}\right) \rho_{1}(h)=p_{2} \rho_{1}(h)$. Since the $G$-action on $P_{2}$ is free, we have $\rho_{1}(h)=\rho_{2}(h)$ for all $h \in H$. This proves the first part.

For the second part, we first show uniqueness of $\varphi$. Suppose a $\Gamma$-equivariant $G$-bundle isomorphism $\varphi: P_{1} \rightarrow P_{2}$ such that $\varphi\left(p_{1}\right)=p_{2}$ exists. Then it must have the property $\varphi\left(u p_{1} g\right)=u p_{2} g$ for all $u \in \Gamma$ and $g \in G$. Since $Z_{1}$ and $Z_{2}$ are transitive $\Gamma$-sets, the actions of $\Gamma \times G$ on $P_{1}$ and $P_{2}$ are transitive. Thus the above identity uniquely determines $\varphi$. This proves uniqueness of $\varphi$.

For existence, since $\rho_{p_{1}}=\rho_{p_{2}}=\rho$, say, we have $P_{1} \underset{\varphi_{1}}{\stackrel{\cong}{\leftrightarrows}} \Gamma \times_{\rho} G \underset{\varphi_{2}}{\stackrel{\cong}{\longrightarrow}} P_{2}$, where $\varphi_{i}$ are $\Gamma$-equivariant $G$-bundle isomorphisms in Lemma 3-2 such that $\varphi_{i}([1,1])=$ $u p_{i} g$ for $i=1,2$. Then $\varphi=\varphi_{2} \circ \varphi_{1}^{-1}$ is the desired $\Gamma-G$ isomorphism. This completes the proof. 
Next, we classify $\Gamma$-equivariant $G$-bundles over transitive $\Gamma$-sets. Let $\pi: P \rightarrow$ $Z$ be such a bundle. For $z \in Z$, let $H_{z}$ be the isotropy subgroup at $z$. Then the collection of homomorphisms $\rho_{p}: H_{z} \rightarrow G$ for all $p \in \pi^{-1}(z)$ forms a complete $G$-conjugacy class, that is, an element in $\operatorname{Hom}\left(H_{z}, G\right) / G$ in view of (3-5). The normalizer $N_{\Gamma}\left(H_{z}\right)$ acts on this set of conjugacy classes by conjugating $H_{z}$. For each point $z \in Z$, we consider the set of $N_{\Gamma}\left(H_{z}\right)$-orbits in $\operatorname{Hom}\left(H_{z}, G\right) / G$. This gives us a bundle of sets:

$$
\omega: \coprod_{z \in Z} \operatorname{Hom}\left(H_{z}, G\right) /\left(N_{\Gamma}\left(H_{z}\right) \times G\right) \rightarrow Z .
$$

This bundle $\omega$ depends only on the $\Gamma$-set $Z$. The above argument shows that for any $\Gamma$-equivariant $G$-bundle $P$ over $Z$, we have an associated section $\eta_{P}$ of $\omega$ whose value at $z \in Z$ is the $N_{\Gamma}\left(H_{z}\right)$-orbit of the $G$-conjugacy class $\left[\rho_{p}\right] \in \operatorname{Hom}\left(H_{z}, G\right) / G$ for any $p \in \pi^{-1}(z)$. Note that for any $P$ over a $\Gamma$ transitive set $Z$, this section $\eta_{P}$ is determined by its value at any single point $z \in Z$, since $\rho_{p}$ for $p \in \pi^{-1}(z)$ determines $\rho_{p}^{\prime}$ for any other point $p^{\prime} \in P$ when $Z$ is $\Gamma$-transitive by (3-5). We show that this section $\eta_{P}$ of the bundle $\omega$, or equivalently, its value at any point of $Z$, determines the $\Gamma-G$ isomorphism class of the bundle $\pi: P \rightarrow Z$ for a $\Gamma$-transitive set $Z$.

Proposition 3-5 Let $\pi_{i}: P_{i} \rightarrow Z$ be $\Gamma$-equivariant $G$ principal bundles over the same transitive $\Gamma$-set $Z$ for $i=1,2$. Then the following statements are equivalent:

(1) Two bundles $P_{1}$ and $P_{2}$ are isomorphic as $\Gamma$-equivariant $G$-principal bundles.

(2) For some $z_{0} \in Z$, we have $\eta_{P_{1}}\left(z_{0}\right)=\eta_{P_{2}}\left(z_{0}\right) \in \operatorname{Hom}\left(H_{z_{0}}, G\right) /\left(N_{\Gamma}\left(H_{z_{0}}\right) \times\right.$ $G)$.

(3) For all $z \in Z$, we have $\eta_{P_{1}}(z)=\eta_{P_{2}}(z) \in \operatorname{Hom}\left(H_{z}, G\right) /\left(N_{\Gamma}\left(H_{z}\right) \times G\right)$.

Proof $(1) \Rightarrow(3)$. Let $\varphi: P_{1} \rightarrow P_{2}$ be a $\Gamma$-equivariant $G$-bundle isomorphism. For any $p_{1} \in P_{1}$, let $p_{2}=\varphi\left(p_{1}\right)$, and $\pi_{1}\left(p_{1}\right)=z_{1}, \pi_{2}\left(p_{2}\right)=z_{2}$. Since the induced $\Gamma$-isomorphism $\bar{\varphi}$ on $Z$ is such that $\bar{\varphi}\left(z_{1}\right)=\left(z_{2}\right)$, the isotropy subgroups at these points are the same $H_{z_{1}}=H_{z_{2}}$, and since $\Gamma$ acts transitively on $Z$, we must have $z_{2}=u z_{1}$ for some $u \in N_{\Gamma}\left(H_{z_{1}}\right)$. To compare $\eta_{P_{1}}$ and $\eta_{P_{2}}$ over the same point $z_{1}$, let $p_{2}^{\prime}=u^{-1} p_{2} g^{-1} \in P_{2}$ for any $g \in G$. Then both $p_{2}^{\prime}$ and $p_{1}$ are points above $z_{1}$. Let $\rho_{i}=\rho_{p_{i}}$ for $i=1,2$, and $\rho_{2}^{\prime}=$ $\rho_{p_{2}^{\prime}}$. Then by (3-5), we have $\rho_{2}\left(u h u^{-1}\right)=g^{-1} \rho_{2}^{\prime}(h) g$ for all $h \in H_{z_{1}}$. Since $\varphi\left(p_{1}\right)=p_{2}$, by Proposition 3-6 we have $\rho_{1}=\rho_{2}$. Thus, we have $\rho_{1}\left(u h u^{-1}\right)=$ $g^{-1} \rho_{2}^{\prime}(h) g$ for all $h \in H_{z_{1}}$. This means that $\eta_{P_{1}}\left(z_{1}\right)=\left[\rho_{1}\right]=\left[\rho_{2}^{\prime}\right]=\eta_{P_{2}}\left(z_{1}\right)$ 
in $\operatorname{Hom}\left(H_{z_{1}}, G\right) /\left(N_{\Gamma}\left(H_{z_{1}}\right) \times G\right)$. Since $p_{1} \in P_{1}$ can be an arbitrary point, we have $\eta_{P_{1}}=\eta_{P_{2}}$ as sections of the bundle $\omega$. This proves (3).

$(3) \Rightarrow(2)$. This is obvious.

$(2) \Rightarrow(1)$. Suppose for some $z_{0} \in Z$, we have $\eta_{P_{1}}\left(z_{0}\right)=\eta_{P_{2}}\left(z_{0}\right)$. this means that for any choices of points $p_{1} \in \pi_{1}^{-1}\left(z_{0}\right)$ and $p_{2} \in \pi_{2}^{-1}\left(z_{0}\right)$, there exists $u \in N_{\Gamma}\left(H_{z_{0}}\right)$ and $g \in G$ such that $\rho_{1}\left(u h u^{-1}\right)=g^{-1} \rho_{2}(h) g$ for all $h \in H_{z_{0}}$. Here we put $\rho_{i}=\rho_{p_{i}}$ for $i=1,2$. Let $p_{2}^{\prime}=u p_{2} g \in P_{2}$. Then, letting $\rho_{2}^{\prime}=\rho_{p_{2}^{\prime}}$, we have $\rho_{2}^{\prime}\left(u h u^{-1}\right)=g^{-1} \rho_{2}(h) g$ for all $h \in H_{z_{0}}$ by (3-5). Thus, we have $\rho_{1}=\rho_{2}^{\prime}: H_{z_{0}} \rightarrow G$. By the second part of Proposition 3-4, there exists a unique $\Gamma$-equivariant $G$-bundle map $\varphi: P_{1} \rightarrow P_{2}$ such that $\varphi\left(p_{1}\right)=p_{2}^{\prime}$. Note that the induced $\Gamma$-map $\bar{\varphi}$ on $Z$ is such that $\bar{\varphi}\left(z_{0}\right)=u z_{0}$, and $z_{0}$ and $u z_{0}$ have the same isotropy subgroups. This completes the proof.

Remark We can also think of the above situation in the following (better) way. When $Z$ is a transitive $\Gamma$-set, we consider a bundle over $Z$ :

$$
\coprod_{z \in Z} \operatorname{Hom}\left(H_{z}, G\right) / G \rightarrow Z \text {. }
$$

Any $\Gamma$-equivariant $G$ bundle $\pi: P \rightarrow Z$ gives rise to a section $s_{P}$ whose value at $z \in Z$ is the $G$-conjugacy class $\left\{\rho_{p}\right\}_{p \in \pi^{-1}(z)}$. Now this section $s_{P}$ is $\Gamma$-equivariant. Here the result of an action of $u \in \Gamma$ on $f \in \operatorname{Hom}(H, G)$ is given by $u f \in \operatorname{Hom}\left(u H u^{-1}, G\right)$ defined by $(u f)\left(u h u^{-1}\right)=f(h)$ for any $h \in H$. Thus dividing by the action of $\Gamma$, each bundle $P$ determines a unique element $\left[s_{P}\right]$ whose representative is given by $\eta_{P}(z) \in \operatorname{Hom}\left(H_{z}, G\right) /\left(N_{\Gamma}\left(H_{z}\right) \times G\right)$ for any $z \in Z$. Proposition 3-5 says that this element classifies the $\Gamma$-equivariant $G$-bundle isomorphism class of $\pi: P \rightarrow Z$.

Reformulating this proposition, we have the classification theorem of $\Gamma$-equivariant $G$-principal bundles over transitive $\Gamma$-sets.

Theorem 3-6 (Classification of $\Gamma-G$ bundles) Let $G$ and $\Gamma$ be any groups. Then there exists the following bijective correspondence:

$\left\{\begin{array}{l}\text { Isomorphism classes of } \Gamma \text {-irreducible } G \text { - } \\ \text { principal bundles over } \Gamma \text {-sets of order } n\end{array}\right\} \stackrel{1: 1}{\longleftrightarrow} \coprod_{[H]_{n}} \operatorname{Hom}(H, G) /\left(N_{\Gamma}(H) \times G\right)$,

where $[H]_{n}$ runs over the set of conjugacy classes of index $n$ subgroups of $\Gamma$.

If we allow $\Gamma$-irreducible $G$-bundles over arbitrary not necessarily finite $\Gamma$-sets, then the bijective correspondence is still valid without the finiteness restriction on $|\Gamma / H|$ on the right hand side. 


\section{Centralizers of homomorphisms into wreath prod- ucts}

Let $\theta: \Gamma \rightarrow G: \mathfrak{S}_{n}=G_{n}$ be a homomorphism into a wreath product. In this section, we determine the structure of the centralizer of the image of $\theta$ in $G_{n}$. Of course this is a purely group theoretic problem, but we found it illuminating and simpler to consider this problem from a geometric point of view.

From the proof of Theorem 3-1, given $\theta$ as above, there exists a $\Gamma$-equivariant $G$-principal bundle $\pi: P \rightarrow Z$ over a $\Gamma$-set $Z$ of order $n$ such that for appropriate choices of a section $s: Z \rightarrow P$ and a bijection $t: \mathbf{n} \stackrel{\cong}{\longrightarrow} Z$, we have the following commutative diagram of groups and homomorphisms.

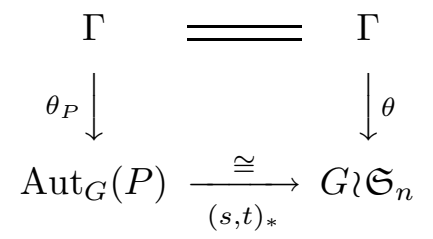

Here the bottom arrow is the one induced by a trivialization $(s, t): \mathbf{n} \times G \cong P$. Now any element in the centralizer $C(\theta) \subset G_{n}$ corresponds, under $(s, t)_{*}$, to a $G$-bundle automorphism of $P$ which commutes with the action $\theta_{P}$ of $\Gamma$ on $P$. These are $\Gamma$-equivariant $G$-bundle automorphisms of $P$ which we denote by $\operatorname{Aut}_{\Gamma-G}(P)$. This proves the following lemma.

Lemma 4-1 Let $\theta: \Gamma \rightarrow G \imath \mathfrak{S}_{n}$ be a homomorphism. Let $\pi: P \rightarrow Z$ be any $\Gamma$-equivariant $G$-bundle whose isomorphism class corresponds to the $G_{n}$-conjugacy class $[\theta]$. Then we have $C(\theta) \cong \operatorname{Aut}_{\Gamma-G}(P)$.

When the base $Z$ is a transitive $\Gamma$-set, we have called $\pi: P \rightarrow Z$ a $\Gamma$-irreducible $G$-bundle. In this case, the group $\Gamma \times G$ acts transitively on the total space $P$. Our next task is to describe $\Gamma-G$ automorphisms of a $\Gamma$-irreducible $G$-bundle. By Lemma 3-2, such a bundle is of the form $\pi_{\rho}: P_{\rho}=\Gamma \times \times_{\rho} G \rightarrow Z=\Gamma / H$ for some subgroup $H \subset \Gamma$ and some homomorphism $\rho: H \rightarrow G$. We have the following obvious exact sequence:

$$
1 \rightarrow \operatorname{Aut}_{\Gamma-G}\left(P_{\rho}\right)_{Z} \rightarrow \operatorname{Aut}_{\Gamma-G}\left(P_{\rho}\right) \rightarrow \operatorname{Aut}_{\Gamma}^{P}(Z) \rightarrow 1 .
$$

Here $\operatorname{Aut}_{\Gamma-G}\left(P_{\rho}\right)_{Z}$ is a subgroup of $\operatorname{Aut}_{\Gamma-G}\left(P_{\rho}\right)$ inducing the identity map on $Z$. The group $\operatorname{Aut}_{\Gamma}^{P}(Z)$ is a subgroup of the $\operatorname{group} \operatorname{Aut}_{\Gamma}(Z) \cong H \backslash N_{\Gamma}(H)$ of $\Gamma$-automorphisms of $Z$ which extend to $\Gamma-G$ automorphisms of $P_{\rho}$. 
Remark Since $H$ is a normal subgroup of $N_{\Gamma}(H)$, it does not matter whether we use right cosets $H \backslash N_{\Gamma}(H)$ or left cosets $N_{\Gamma}(H) / H$ : they are the same group. We use right cosets notation here only because it is more appropriate due to the formula of the isomorphism $\Phi: H \backslash N_{\Gamma}(H) \cong \operatorname{Aut}_{\Gamma}(Z)$ in the proof of Theorem $4-2$.

For $\rho: H \rightarrow G$ as above and $u \in N_{\Gamma}(H)$, let $\rho^{u}: H \rightarrow G$ be a homomorphism defined by conjugation by $u$, that is, $\rho^{u}(h)=\rho\left(u h u^{-1}\right)$ for $h \in H$. Let

$$
N_{\Gamma}^{\rho}(H)=\left\{u \in N_{\Gamma}(H) \mid \rho^{u} \text { and } \rho \text { are } G \text {-conjugate }\right\} .
$$

This subset of $N_{\Gamma}(H)$ is easily seen to be a subgroup of $N_{\Gamma}(H)$. Another way to think about $N_{\Gamma}^{\rho}(H)$ is as the isotropy subgroup at $[\rho]$ of the $N_{\Gamma}(H)$-action on $\operatorname{Hom}(H, G) / G$.

Theorem 4-2 For $\rho: H \rightarrow G$, let $\pi_{\rho}: P_{\rho} \rightarrow Z(=\Gamma / H)$ be the associated $\Gamma$ irreducible $G$-bundle. Then $\operatorname{Aut}_{\Gamma-G}\left(P_{\rho}\right)_{Z} \cong C_{G}(\rho)$ and $\operatorname{Aut}_{\Gamma}^{P}(Z) \cong H \backslash N_{\Gamma}^{\rho}(H)$. Thus, the group of $\Gamma-G$ automorphisms of $P_{\rho}$ fits into the following exact sequence:

$$
1 \rightarrow C_{G}(\rho) \rightarrow \operatorname{Aut}_{\Gamma-G}\left(P_{\rho}\right) \rightarrow H \backslash N_{\Gamma}^{\rho}(H) \rightarrow 1 .
$$

Let $p_{0}=[1,1] \in P_{\rho}$. For any $g^{\prime} \in C_{G}(\rho)$, the corresponding $\Gamma-G$ automorphism $\varphi_{g^{\prime}}$ of $P_{\rho}$ is given by $\varphi_{g^{\prime}}\left(u p_{0} g\right)=u p_{0} g^{\prime-1} g$ for any $u \in \Gamma$ and $g \in G$.

Proof First note that when $g^{\prime} \in C(\rho)$, we have $\rho_{p_{0} g^{\prime-1}}=g^{\prime} \rho_{p_{0}} g^{\prime-1}=\rho_{p_{0}}$, where $\rho_{p_{0}}=\rho$. Hence by Proposition 3-4, the map $\varphi_{g^{\prime}}$ given above is indeed a $\Gamma$-equivariant $G$-bundle isomorphism inducing the identity map on the base $Z$. This gives us a map $C(\rho) \rightarrow \operatorname{Aut}_{\Gamma-G}\left(P_{\rho}\right)_{Z}$. This can be easily checked to be an injective homomorphism directly from the definition of $\varphi_{g^{\prime}}$. To see that this is a surjective map, let $\varphi: P_{\rho} \rightarrow P_{\rho}$ be a $\Gamma$-equivariant $G$-bundle automorphism of $P_{\rho}$ inducing the identity map on $Z$. Then $\varphi\left(p_{0}\right)$ is a point in the same fibre. So $\varphi\left(p_{0}\right)=p_{0} g^{-1}$ for some unique $g^{\prime} \in G$. By Proposition 3-4, we must have $\rho_{p_{0}}=\rho_{p_{0} g^{\prime-1}}$. By (3-5), this means that $g^{\prime} \rho_{p_{0}}(h) g^{\prime-1}=\rho_{p_{0}}(h)$ for any $h \in H$. Thus, $g^{\prime} \in C(\rho)$. This proves that $C(\rho) \cong \operatorname{Aut}_{\Gamma-G}\left(P_{\rho}\right)_{Z}$.

Let $z_{0}=[H] \in \Gamma / H$. Recall that the isomorphism $\Phi: H \backslash N_{\Gamma}(H) \cong \operatorname{Aut}_{\Gamma}(Z)$ is given by $\Phi\left(H u^{\prime}\right)\left(u z_{0}\right)=u u^{\prime-1} z_{0}$. If $u^{\prime} \in N_{\Gamma}^{\rho}(H)$, then by definition (4-1) there exists $g^{\prime} \in G$ such that $\rho\left(u^{\prime-1} h u^{\prime}\right)=g^{\prime-1} \rho(h) g^{\prime}$ for any $h \in H$. This means $\rho_{p_{0}}=\rho_{u^{\prime-1}} p_{0} g^{\prime}$ by $(3-5)$, where $p_{0}=[1,1] \in P_{\rho}$. Then by Proposition 3 -4, there exists a unique $\Gamma$-equivariant $G$-bundle isomorphism $\varphi: P \rightarrow P$ such that $\varphi\left(u p_{0} g\right)=u\left(u^{\prime-1} p_{0} g^{\prime}\right) g$ for $u \in \Gamma$ and $g \in G$. The induced $\Gamma$ map on the base $Z$ is $\bar{\varphi}\left(u z_{0}\right)=u u^{\prime-1} z_{0}$. Thus, any element in the image of 
the subgroup $H \backslash N_{\Gamma}^{\rho}(H)$ under $\Phi$ lifts to a $\Gamma$-G-map of $P$. There are $|C(\rho)|$ many choices of such lifts. Thus by restriction, we have a well defined injective homomorphism $\Phi^{\prime}: H \backslash N_{\Gamma}^{\rho}(H) \rightarrow \operatorname{Aut}_{\Gamma}^{P}(Z)$. To see that this map is surjective, let $\bar{\varphi}: Z \rightarrow Z$ be a $\Gamma$-equivariant isomorphism induced from a $\Gamma-G$ map $\varphi: P_{\rho} \rightarrow P_{\rho}$. Suppose $\varphi\left(p_{0}\right)=u^{\prime-1} p_{0} g^{\prime}$ for some $u^{\prime} \in \Gamma$ and $g^{\prime} \in G$. Then the $\Gamma$-map $\bar{\varphi}$ on $Z$ is given by $\bar{\varphi}\left(u z_{0}\right)=u u^{\prime-1} z_{0}$. By Proposition 3-4, $\rho_{p_{0}}=\rho_{u^{\prime-1}} p_{0} g^{\prime}$. This means $\rho\left(u^{\prime-1} h u^{\prime}\right)=g^{\prime-1} \rho(h) g^{\prime}$ for all $h \in H$ by $(3-5)$. This in turn means that $u^{\prime} \in N_{\Gamma}^{\rho}(H)$ by (4-1). Hence we have $\bar{\varphi}=\Phi^{\prime}\left(H u^{\prime}\right)$, and this shows that $\Phi^{\prime}$ is surjective. Thus $\Phi^{\prime}$ is an isomorphism of groups. This completes the proof.

We give another description of $\operatorname{Aut}_{\Gamma}^{P}(Z)$. Recall that given a $\Gamma$-equivariant $G$-bundle $\pi: P \rightarrow Z$, we have a section $s_{P}$ of the bundle (3-8) over $Z$. Let $D_{P}(Z)$ be the set of all $\Gamma$-automorphisms of $Z$ which leaves $s_{P}$ invariant:

$$
D_{P}(Z)=\left\{\tau: Z \rightarrow Z \mid \tau \text { is } \Gamma \text {-equivariant bijection and } s_{P} \circ \tau=s_{P}\right\} .
$$

We show that any element in $D_{P}(Z)$ extends to a $\Gamma$-equivariant $G$-bundle map of $P$. For the next proposition, we do not have to assume that $Z$ is a transitive $\Gamma$-set.

Proposition 4-3 Let $\pi: P \rightarrow Z$ be a $\Gamma$-equivariant $G$-principal bundle over a (not necessarily transitive) $\Gamma$-set $Z$. With the above notations, we have $\operatorname{Aut}_{\Gamma}^{P}(Z)=D_{P}(Z)$.

Proof Let $\varphi: P \stackrel{\cong}{\longrightarrow} P$ be a $\Gamma$-equivariant $G$-bundle automorphism. We show that the induced map $\bar{\varphi}$ on the base is in $D_{P}(Z)$. Since $\bar{\varphi}$ is a $\Gamma$-map, for any $p \in P$, isotropy subgroups at $\pi(\varphi(p))$ and $\pi(p)$ are the same. By Proposition 3 -4 we have $\rho_{\varphi(p)}=\rho_{p}$. Now, $s_{P} \circ \bar{\varphi}(\pi(p))=\left[\rho_{\varphi(p)}\right]=\left[\rho_{p}\right]=s_{P}(\pi(p))$ for any $p \in P$. Thus $s_{P} \circ \bar{\varphi}=s_{P}$, and $\bar{\varphi} \in D_{P}(Z)$. This proves $\operatorname{Aut}_{\Gamma}^{P}(Z) \subseteq D_{P}(Z)$.

For the other direction of inclusion, let $\tau: Z \rightarrow Z$ be a $\Gamma$-map such that $s_{P} \circ \tau=s_{P}$. Let $Z=\coprod_{\xi} Z_{\xi}$ be the $\Gamma$-orbit decomposition of $Z$, and let the corresponding decomposition of $\Gamma-G$ bundles be $(P \rightarrow Z)=\coprod_{\xi}\left(P_{\xi} \rightarrow Z_{\xi}\right)$. The $\Gamma$-map $\tau$ permutes orbits of the same type. We pick any point $z \in Z$, say, $z \in Z_{\xi}$. Let $\tau(z)=z^{\prime} \in Z_{\xi^{\prime}}$. Thus isotropy subgroups at $z$ and $z^{\prime}$ are the same, say $H$. Since $s_{P} \circ \tau(z)=s_{P}(z)$, for any points $p \in \pi^{-1}(z)$ and $p^{\prime} \in \pi^{-1}\left(z^{\prime}\right)$ we have $\left[\rho_{p}\right]=\left[\rho_{p^{\prime}}\right]$ in $\operatorname{Hom}(H, G) / G$. Thus there exists $g \in G$ such that $\rho_{p^{\prime}}=g \rho_{p} g^{-1}$. By (3-5), letting $p^{\prime \prime}=p^{\prime} g$ we have $\rho_{p^{\prime \prime}}=\rho_{p}$. Hence by Proposition 3-4, there exists a unique $\Gamma$-equivariant $G$-bundle map $\varphi_{\xi}: P_{\xi} \rightarrow P_{\xi^{\prime}}$ such that $\varphi_{\xi}(p)=p^{\prime \prime}$. Since the induced $\Gamma$-map on the base is such that $\bar{\varphi}_{\xi}(z)=\pi\left(p^{\prime \prime}\right)=z^{\prime}=\tau(z)$ and both $Z_{\xi}$ and $Z_{\xi^{\prime}}$ are transitive 
$\Gamma$-sets, we have $\bar{\varphi}_{\xi}=\left.\tau\right|_{Z_{\xi}}: Z_{\xi} \rightarrow Z_{\xi^{\prime}}$. We repeat this construction for the remaining $Z_{\xi}$ 's, and we get a $\Gamma$-equivariant $G$-bundle map $\varphi: P \rightarrow P$ lifting $\tau$. Hence $\tau \in \operatorname{Aut}_{\Gamma}^{P}(Z)$. This completes the proof.

Next we give an alternate description of the structure of the group $\operatorname{Aut}_{\Gamma-G}\left(P_{\rho}\right)$ when the base set of $P$ is a transitive $\Gamma$-set. This description is more direct than the one in Theorem 4-2. We take the point of view that $(u, g) \in \Gamma \times G$ acts on $p \in P_{\rho}$ from the right by $p \cdot(u, g)=u^{-1} p g$. The isotropy subgroup at $p_{0}=[1,1] \in P_{\rho}$ is $\{(h, \rho(h)) \mid h \in H\} \cong H$, so that as a right $\Gamma \times G$-set, $P_{\rho} \cong H \backslash(\Gamma \times G)$ using right cosets.

By Proposition 3-4, we have a bijection $P_{\rho} \supset\left\{p \in P_{\rho} \mid \rho_{p}=\rho\right\} \stackrel{\cong}{\longrightarrow} \operatorname{Aut}_{\Gamma-G}\left(P_{\rho}\right)$ as sets. When $p=u^{-1} p_{0} g$, by (3-5) we have $\rho_{p}(h)=g^{-1} \rho\left(u h u^{-1}\right) g$ for all $h \in H$. We consider a subset $T_{\rho}$ of $N_{\Gamma}(H) \times G \subset \Gamma \times G$ consisting of pairs $(u, g)$ such that the corresponding point $p=u^{-1} p_{0} g$ has the property that $\rho_{p}=\rho$. Namely, let

$$
T_{\rho}=\left\{(u, g) \in N_{\Gamma}(H) \times G \mid g^{-1} \rho\left(u h u^{-1}\right) g=\rho(h), \text { for all } h \in H\right\} .
$$

From this definition, we see that if $(u, g) \in T_{\rho}$, then it follows that $u \in N_{\Gamma}^{\rho}(H)$, in view of its definition (4-1). We can easily check that $T_{\rho}$ is a subgroup of $N_{\Gamma}(H) \times G$.

Another way to look at $T_{\rho}$ is as follows. Let $(u, g) \in N_{\Gamma}(H) \times G$ act from the right on an element $f \in \operatorname{Hom}(H, G)$ by $[f \cdot(u, g)](h)=g^{-1} f\left(u h u^{-1}\right) g$ for $h \in H$. Then, $T_{\rho} \subset N_{\Gamma}(H) \times G$ is the isotropy subgroup at $\rho$. This makes it clear that $T_{\rho}$ is indeed a subgroup.

We have the following diagram:

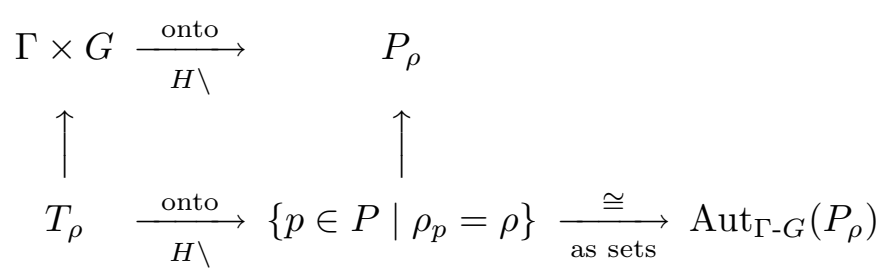

where all vertical maps are inclusions. This shows that $H \backslash T_{\rho} \cong \operatorname{Aut}_{\Gamma-G}\left(P_{\rho}\right)$ as sets. The correspondence is the following one. For $(u, g) \in T_{\rho}$, it corresponds to a point $u^{-1} p_{0} g \in P_{\rho}$. This point corresponds to a $\Gamma$-equivariant $G$-bundle automorphism $\varphi_{(u, g)}$ of $P_{\rho}$ characterized by $\varphi_{(u, g)}\left(p_{0}\right)=u^{-1} p_{0} g$ by Proposition 3 -4. In fact, this bijective correspondence is as groups. 
Theorem 4-4 Let an injective homomorphism $\iota: H \rightarrow T_{\rho}$ be given by $\iota(h)=(h, \rho(h))$ for $h \in H$. Then, $\operatorname{Im} \iota$ is a normal subgroup of $T_{\rho}$, and the group of $\Gamma$-equivariant $G$-bundle automorphism of $P_{\rho}$ fits into the following exact sequence of groups:

$$
1 \rightarrow H \stackrel{\iota}{\rightarrow} T_{\rho} \stackrel{j}{\rightarrow} \operatorname{Aut}_{\Gamma-G}\left(P_{\rho}\right) \rightarrow 1 .
$$

Here, for $(u, g) \in T_{\rho}$, its image $j((u, g))=\varphi_{(u, g)}$ is a $\Gamma$-equivariant $G$-bundle automorphism of $P_{\rho}$ given by $\varphi_{(u, g)}([v, k])=\left[v u^{-1}, g k\right]$ for any $v \in \Gamma$ and $k \in G$. In particular, $H \backslash T_{\rho} \cong \operatorname{Aut}_{\Gamma-G}\left(P_{\rho}\right)$ as groups.

Proof For the first part, for any $h \in H$ and any $(u, g) \in T_{\rho}$, we have

$$
(u, g)(h, \rho(h))(u, g)^{-1}=\left(u h u^{-1}, g \rho(h) g^{-1}\right)=\left(u h u^{-1}, \rho\left(u h u^{-1}\right)\right),
$$

where the second equality holds because $(u, g) \in T_{\rho}$. Since $u \in N_{\Gamma}(H)$, we have $u h u^{-1} \in H$. This proves that the isomorphic image of $H$ under $\iota$ in $T_{\rho}$ is a normal subgroup.

For the second part, it is straightforward to check that the map $j$ described above is a homomorphism with kernel $\iota(H)$. Since the bijective correspondence $H \backslash T_{\rho} \cong \operatorname{Aut}_{\Gamma-G}\left(P_{\rho}\right)$ described earlier is precisely the one induced by $j$ above, this correspondence is an isomorphism of groups. This completes the proof.

We also have a related exact sequence involving $T_{\rho}$. Almost by definition (4-4), we have $T_{\rho} \subset N_{\Gamma}^{\rho}(H) \times G$. Projection to $N_{\Gamma}^{\rho}(H)$ is onto by definition (4-1), and we have the following exact sequence of groups:

$$
1 \rightarrow C_{G}(\rho) \rightarrow T_{\rho} \rightarrow N_{\Gamma}^{\rho}(H) \rightarrow 1 .
$$

Dividing by the left action by $H$, we recover (4-2). Here, note that $H \cap C(\rho)$ is trivial in $T_{\rho}$.

Theorem 4-2 and Theorem 4-4 deal with the group of $\Gamma$-equivariant $G$-bundle automorphisms of a $\Gamma$-irreducible $G$-bundle $P_{\rho}$. Next we describe the $\Gamma$ - $G$ automorphism group of a general $\Gamma$-equivariant $G$-bundle $\pi: P \rightarrow Z$, where the base set $Z$ is not necessarily a transitive $\Gamma$-set, nor a finite $\Gamma$-set. Any such bundle decomposes into a disjoint union of $\Gamma$-irreducible $G$-bundles, and these irreducible bundles are classified by Theorem 3-6. For each conjugacy class $[H]$ of subgroups of $\Gamma$, and for each conjugacy class $[\rho] \in \operatorname{Hom}(H, G) /\left(N_{\Gamma}(H) \times G\right)$, let $r(H, \rho)$ be the number of isomorphic copies of $P_{\rho}=\Gamma \times{ }_{\rho} G \rightarrow \Gamma / H$ appearing in the decomposition of $\pi: P \rightarrow Z$. Let $P[H, \rho]$ be the sub-bundle of $P$ of type $([H],[\rho])$. Thus $P[H, \rho]$ is isomorphic to a disjoint union of $r(H, \rho)$ copies of $\pi_{\rho}: P_{\rho} \rightarrow \Gamma / H$. If $([H],[\rho]) \neq\left(\left[H^{\prime}\right],\left[\rho^{\prime}\right]\right)$, then there are no $\Gamma$ - $G$ isomorphisms between $P[H, \rho]$ and $P\left[H^{\prime}, \rho^{\prime}\right]$. Furthermore, it is elementary that

$$
\operatorname{Aut}_{\Gamma-G}(P[H, \rho]) \cong \operatorname{Aut}_{\Gamma-G}\left(P_{\rho}\right) \imath \mathfrak{S}_{r(H, \rho)} .
$$

Combining these results, we finally obtain the structure theorem for $\operatorname{Aut}_{\Gamma-G}(P)$. 
Theorem 4-5 (Structure of $\Gamma-G$ automorphism groups and centralizers) Let $\pi: P \rightarrow Z$ be a $\Gamma$-equivariant $G$-principal bundle over a $\Gamma$-set $Z$. Let $r(H, \rho)$ be the number of isomorphic copies of $P_{\rho}=\Gamma \times{ }_{\rho} G \rightarrow \Gamma / H$ appearing in the irreducible decomposition of $P \rightarrow Z$. Then,

$$
\operatorname{Aut}_{\Gamma-G}(P) \cong \prod_{[H]} \prod_{[\rho]} \operatorname{Aut}_{\Gamma-G}\left(P_{\rho}\right)\left\langle\mathfrak{S}_{r(H, \rho)},\right.
$$

where $[H]$ runs over all conjugacy classes of subgroups of $\Gamma$, and for a given $[H]$, $[\rho]$ runs over the set $\operatorname{Hom}(H, G) /\left(N_{\Gamma}(H) \times G\right)$. The structure of $\operatorname{Aut}_{\Gamma-G}\left(P_{\rho}\right)$ is described in Theorem 4-2 and Theorem 4-4.

For a given homomorphism $\theta: \Gamma \rightarrow G \imath \mathfrak{S}_{n}$, let $\pi: P \rightarrow Z$ be a $\Gamma-G$ bundle whose isomorphism class corresponds to $[\theta]$ under the correspondence (3-4). Then the centralizer $C_{G_{n}}(\theta)$ is isomorphic to $\operatorname{Aut}_{\Gamma-G}(P)$ given in (4-7).

\section{Generating functions of orbifold Euler character- istics of symmetric products: exponential formulae}

In this section, we let $G$ be a finite group and let $M$ be a $G$-manifold. Let $\varphi(M ; G)$ be an arbitrary multiplicative orbifold invariant of $(M ; G)$. Let $\Gamma$ be an arbitrary group. We are interested in calculating the generating function $\sum_{n \geq 0} q^{n} \varphi_{\Gamma}\left(M^{n} ; G_{n}\right)$ of the $\Gamma$-extended orbifold invariant of symmetric products of an orbifold. Here $G_{n}=G \imath \mathfrak{S}_{n}$ is a wreath product and

$$
\varphi_{\Gamma}\left(M^{n} ; G_{n}\right)=\sum_{[\theta] \in \operatorname{Hom}\left(\Gamma, G_{n}\right) / G_{n}} \varphi\left(\left(M^{n}\right)^{\langle\theta\rangle} ; C_{G_{n}}(\theta)\right) .
$$

Let $\theta: \Gamma \rightarrow G_{n}=G \imath \mathfrak{S}_{n}$ be a homomorphism. Calculating the above invariant involves identifying fixed point subset $\left(M^{n}\right)^{\langle\theta\rangle}$, and calculating the centralizer $C_{G_{n}}(\theta)$ together with its action on the fixed point subset. These tasks can be done geometrically using $\Gamma$-equivariant $G$-principal bundles.

Given $\theta$ as above, let $\pi_{\theta}: P \rightarrow \mathbf{n}$ be a $\Gamma$-equivariant $G$-bundle over a set $\mathbf{n}=\{1,2, \ldots, n\}$ equipped with a section $s_{\theta}: \mathbf{n} \rightarrow P$ such that the natural action $\theta_{P}$ of $\Gamma$ on $P$ corresponds to $\theta$ through the $G$-bundle trivialization $s: \mathbf{n} \times G \stackrel{\cong}{\longrightarrow} P$ induced by $s_{\theta}$. Note that the $\Gamma$-action on $\mathbf{n}$ comes from $\theta$ via the composition with a projection $\Gamma \rightarrow G$ ᄀ $\mathfrak{S}_{n} \rightarrow \mathfrak{S}_{n}=\operatorname{Aut}(\mathbf{n})$. Such a $\Gamma-G$ bundle $\pi_{\theta}: P \rightarrow \mathbf{n}$ together with section $s_{\theta}$ was constructed in the proof of Theorem 3-1 in two different ways. Let $\mathcal{S}\left[P \times_{G} M\right]$ be the set of sections of the fibre bundle $\pi_{M}: P \times_{G} M \rightarrow \mathbf{n}$. This set can be identified with the set of $G$-equivariant maps $\alpha \in \operatorname{Map}_{G}(P, M)$ satisfying $\alpha(p g)=g^{-1} \alpha(p)$ 
for all $g \in G$ and $p \in P$. A $G$-automorphism $\varphi \in \operatorname{Aut}_{G}(P)$ acts on $\alpha \in$ $\operatorname{Map}_{G}(P, M)$ by $\varphi(\alpha)=\left(\varphi^{-1}\right)^{*}(\alpha)=\alpha \circ \varphi^{-1}$. Thus, through $\theta_{P}, u \in \Gamma$ acts on $\operatorname{Map}_{G}(P, M)=\mathcal{S}\left[P \times_{G} M\right]$ as $\theta_{P}\left(u^{-1}\right)^{*}$. On the other hand, we have an identification $\operatorname{Map}_{G}(\mathbf{n} \times G, M) \stackrel{\cong}{\rightrightarrows} M^{n}$ by evaluation at $1 \in G$. Since $u \in \Gamma$ acts on $\mathbf{n} \times G$ as $\theta(u)$, the action of $u \in \Gamma$ on $\operatorname{Map}_{G}(\mathbf{n} \times G, M) \cong M^{n}$ is given by $\theta\left(u^{-1}\right)^{*}$. As we remarked earlier after Lemma 3-3, this action of $\Gamma$ on $M^{n}$ is precisely the one given in (3-2). Since $s: P \rightarrow \mathbf{n} \times G$ is $\Gamma$-equivariant with respect to $\theta_{P}$ and $\theta$, we see that the $\Gamma$ action on $\mathcal{S}\left[P \times{ }_{G} M\right]$ corresponds precisely to the $\Gamma$ action on $M^{n}$. The situation can be summarized by the following diagrams.

$$
\begin{array}{ccc}
\mathcal{S}\left[P \times_{G} M\right] \cong \operatorname{Map}_{G}(P, M) & \underset{\theta_{P}\left(u^{-1}\right)^{*}}{\cong} & \operatorname{Map}_{G}(P, M) \cong \mathcal{S}\left[P \times_{G} M\right] \\
\cong s^{*} & \cong \downarrow s^{*} \\
M^{n} \cong \operatorname{Map}_{G}(\mathbf{n} \times G, M) & \underset{\theta\left(u^{-1}\right)^{*}=u .}{\cong} & \operatorname{Map}_{G}(\mathbf{n} \times G, M) \cong M^{n}
\end{array}
$$

Here, horizontal arrows denote actions of $u \in \Gamma$. From this, the first part of the next proposition is clear.

Proposition 5-1 Let $\theta: \Gamma \rightarrow G \backslash \mathfrak{S}_{n}$ be a homomorphism. Let $\pi: P \rightarrow Z$ be a $\Gamma$-equivariant $G$-principal bundle whose isomorphism class corresponds to the conjugacy class $[\theta]$. Then we have $\left(M^{n}\right)^{\langle\theta\rangle} \cong \mathcal{S}\left[P \times_{G} M\right]^{\Gamma}$, where the right hand side is the set of $\Gamma$-fixed sections.

Let $\pi_{\rho}: P_{\rho}=\Gamma \times{ }_{\rho} G \rightarrow \Gamma / H$ be an irreducible $\Gamma$ - $G$-bundle. Then we have $\mathcal{S}\left[P_{\rho} \times{ }_{G} M\right]^{\Gamma} \cong M^{\langle\rho\rangle}$.

Proof We only have to prove the second part. Any section of $P_{\rho} \times_{G} M \rightarrow \Gamma / H$ is represented by a $G$-equivariant map $\alpha: P_{\rho} \rightarrow M$ satisfying $\alpha(p g)=g^{-1} \alpha(p)$ for all $p \in P_{\rho}$ and $g \in G$. This section is $\Gamma$-invariant if $\alpha\left(u^{-1} p\right)=\alpha(p)$ for all $u \in \Gamma$ and $p \in P_{\rho}$. Since $\Gamma$ acts transitively on the base $\Gamma / H$, such a $\Gamma$-invariant $G$-equivariant map $\alpha$ is uniquely determined by its value at $p_{0}=[1,1] \in P_{\rho}$. This value $\alpha\left(p_{0}\right) \in M$ cannot be any point in $M$. For any $h \in H \subset \Gamma$, using $\Gamma$-invariance of $\alpha$, we must have $\alpha\left(p_{0}\right)=\alpha\left(h p_{0}\right)=\alpha\left(p_{0} \rho(h)\right)=\rho(h)^{-1} \alpha\left(p_{0}\right)$. Thus, $\alpha\left(p_{0}\right)$ must belong to $\rho(H)$-invariant subset of $M$. This gives us an injective map $\mathcal{S}\left[P_{\rho} \times_{G} M\right]^{\Gamma} \rightarrow M^{\langle\rho\rangle}$.

To see that this map is surjective, let $x \in M^{\langle\rho\rangle}$ be any $\langle\rho\rangle$-fixed point in $M$. Let a $\Gamma$-invariant $G$-equivariant map $\alpha_{x}: P_{\rho} \rightarrow M$ be defined by a formula $\alpha_{x}\left(u^{-1} p_{0} g\right)=g^{-1} x$ for all $u \in \Gamma$ and $g \in G$. To see that this well defined, let $u_{1}^{-1} p_{0} g_{1}=u_{2}^{-1} p_{0} g_{2}$. Then there exists a unique $h \in H$ such that $u_{2}=h u_{1}$ 
and $g_{2}=\rho(h) g_{1}$. Then $g_{2}^{-1} x=g_{1}^{-1} \rho(h)^{-1} x=g_{1}^{-1} x$, since $x \in M^{\langle\rho\rangle}$. This proves that $\alpha_{x}$ is well defined and the above correspondence is a bijection. This completes the proof.

From this proposition, the next corollary is straightforward.

Corollary 5-2 Let $r(H, \rho)$ be the number of isomorphic copies of $\pi_{\rho}: P_{\rho} \rightarrow$ $\Gamma / H$ appearing in the irreducible decomposition of a $\Gamma$-equivariant $G$-principal bundle $\pi: P \rightarrow Z$. Then

$$
\mathcal{S}\left[P \times_{G} M\right]^{\Gamma} \cong \prod_{[H]} \prod_{[\rho]}\left(M^{\langle\rho\rangle}\right)^{r(H, \rho)} .
$$

Furthermore, the action of $\operatorname{Aut}_{\Gamma-G}(P)$ on the above set respects $[H]-[\rho]$ product decomposition described in (4-7).

Proof Let $\left.[P \rightarrow Z]=\coprod_{[H]} \coprod_{[\rho]}^{r(H, \rho)} \coprod^{\left[\left(P_{\rho}\right.\right.} \rightarrow \Gamma / H\right]$ be the irreducible decomposition of $P \rightarrow Z$. Since the $\Gamma$-action respects the decomposition of $P \rightarrow Z$ into irreducible bundles, we have

$$
\mathcal{S}\left[P \times_{G} M\right]^{\Gamma} \cong \prod_{[H]} \prod_{[\rho]}^{r(H, \rho)} \prod^{\mathcal{S}} \mathcal{S}\left[P_{\rho} \times_{G} M\right]^{\Gamma} .
$$

From the second part of Proposition 5-1, the isomorphism (5-2) follows.

Since there are no $\Gamma-G$ isomorphisms between $P_{\rho} \rightarrow \Gamma / H$ and $P_{\rho^{\prime}} \rightarrow \Gamma / H^{\prime}$ if $([H],[\rho]) \neq\left(\left[H^{\prime}\right],\left[\rho^{\prime}\right]\right)$ by Classification Theorem 3-6, the action of the $\Gamma-G$ automorphism group $\operatorname{Aut}_{\Gamma-G}(P)$ respects $[H]-[\rho]$ decomposition. This completes the proof.

Next we describe the action of $\operatorname{Aut}_{\Gamma-G}\left(P_{\rho}\right)$ on $M^{\langle\rho\rangle}$. By Theorem 4-4, an arbitrary element in $\operatorname{Aut}_{\Gamma-G}\left(P_{\rho}\right)$ is of the form $\varphi_{(u, g)}$ for some $(u, g) \in T_{\rho}$. This pair $(u, g)$ is unique up to the left action by $H$, in view of Theorem 4-4.

Proposition 5-3 The left action of $\varphi_{(u, g)} \in \operatorname{Aut}_{\Gamma-G}\left(P_{\rho}\right)$ on $x \in M^{\langle\rho\rangle} \cong$ $\mathcal{S}\left[P_{\rho} \times{ }_{G} M\right]^{\Gamma}$ is given by $\varphi_{(u, g)}(x)=g x$. 
Proof By definition, the left action of $\varphi \in \operatorname{Aut}_{\Gamma-G}\left(P_{\rho}\right)$ on $\alpha \in \operatorname{Map}_{G}\left(P_{\rho}, M\right) \cong$ $\mathcal{S}\left[P_{\rho} \times_{G} M\right]$ is given by the composition $\varphi(\alpha)=\alpha \circ \varphi^{-1}$. For any $x \in M^{\langle\rho\rangle}$, let $\alpha_{x}: P_{\rho} \rightarrow M$ be the $\Gamma$-invariant $G$-equivariant map such that $\alpha_{x}\left(p_{0}\right)=x$. When $(u, g) \in T_{\rho}$, we have $g^{-1} \rho\left(u h u^{-1}\right) g=\rho(h)$ for any $h \in H$. Since $u \in N_{\Gamma}(H)$, we have $u h u^{-1} \in H$. Since the correspondence $\operatorname{Map}_{G}\left(P_{\rho}, M\right) \cong$ $M^{\langle\rho\rangle}$ is given by evaluation at $p_{0} \in P_{\rho}$, we evaluate $\varphi_{(u, g)}\left(\alpha_{x}\right)=\alpha_{x} \circ \varphi_{(u, g)}^{-1}$ at $p_{0}$. Here $\Gamma-G$ map $\varphi_{(u, g)}$ is characterized by $\varphi_{(u, g)}\left(p_{0}\right)=u^{-1} p_{0} g$. Thus $\varphi_{(u, g)}^{-1}\left(p_{0}\right)=u p_{0} g^{-1}$. Now, $\alpha_{x} \circ \varphi_{(u, g)}^{-1}\left(p_{0}\right)=\alpha_{x}\left(u p_{0} g^{-1}\right)=g \alpha_{x}\left(p_{0}\right)=g x$. Hence the action of $\varphi_{(u, g)}$ on $x$ is given by $\varphi_{(u, g)}(x)=g x$. Indeed, $g x \in M^{\langle\rho\rangle}$ because for any $h \in H, \rho(h)(g x)=g\left(g^{-1} \rho(h) g\right) x=g \rho\left(u^{-1} h u\right) x=g x$, since $x \in M^{\langle\rho\rangle}$. This completes the proof.

Note that when $(u, g) \in T_{\rho}$, the element $g \in G$ is not necessarily in $C(\rho)$, rather it is in the normalizer $N_{G}(\rho(H))$, which can be seen from the definition (4-4) of $T_{\rho}$. In general, the subgroup $\left\{g \in G \mid(u, g) \in T_{\rho}\right\}$ is a proper subgroup of $N_{G}(\rho(H))$. The last part of the above proof says that $N_{G}(\rho(H))$ acts on the $\langle\rho\rangle$-fixed point subset $M^{\langle\rho\rangle}$.

We now calculate the generating function of the invariants $\varphi_{\Gamma}\left(M^{n} ; G_{n}\right)$ given in (5-1) for $n \geq 0$. An actual explicit concise formula depends on individual properties of $\varphi$. However, the next formula applies to any $\varphi$.

Proposition 5-4 Let $\varphi(M ; G)$ be a multiplicative orbifold invariant of a $G$ manifold $M$. Then

$$
\sum_{n \geq 0} q^{n} \varphi_{\Gamma}\left(M^{n} ; G \imath \mathfrak{S}_{n}\right)=\prod_{[H]} \prod_{[\rho]}\left[\sum_{r \geq 0} q^{|\Gamma / H| r} \varphi\left(\left(M^{\langle\rho\rangle}\right)^{r} ; \operatorname{Aut}_{\Gamma-G}\left(P_{\rho}\right)\left\langle\mathfrak{S}_{r}\right)\right]\right.
$$

where $[H]$ runs over the set of all the conjugacy classes of subgroups of $\Gamma$, and for each given $[H],[\rho]$ runs over the set $\operatorname{Hom}(H, G) /\left(N_{\Gamma}(H) \times G\right)$.

Proof First, we rewrite the right hand side of (5-1) in terms of $\Gamma$-equivariant $G$-bundles. By Theorem 3-1, each conjugacy class $[\theta] \in \operatorname{Hom}\left(\Gamma, G_{n}\right) / G_{n}$ corresponds to an isomorphism class of $\Gamma$-equivariant $G$-principal bundle $P$ over a $\Gamma$-set $Z$ of order $n$. Furthermore, using Lemma 4-1, Theorem 4-5, Proposition $5-1$, and Corollary 5-2, we have

$$
\begin{aligned}
\varphi_{\Gamma}\left(M^{n} ; G_{n}\right) & =\sum_{[P]} \varphi\left(\mathcal{S}\left[P \times_{G} M\right]^{\Gamma} ; \operatorname{Aut}_{\Gamma-G}(P)\right) \\
& =\sum_{\{r \mid=n} \prod_{\{r(H, \rho)\}} \prod_{[H][\rho]} \varphi\left(\left(M^{\langle\rho\rangle}\right)^{r(H, \rho)} ; \operatorname{Aut}_{\Gamma-G}\left(P_{\rho}\right)\left\langle\mathfrak{S}_{r(H, \rho)}\right),\right.
\end{aligned}
$$


where the second summation is over all possible sets of non-negative integers $\{r(H, \rho)\}_{[H],[\rho]}$ such that $\sum_{[H],[\rho]}|\Gamma / H| r(H, \rho)=n$. Taking the summation over all $n \geq 0$, we have

$$
\begin{aligned}
\sum_{n \geq 0} q^{n} \varphi_{\Gamma}\left(M^{n} ; G_{n}\right) & =\sum_{r(H, \rho) \geq 0} \prod_{[H]} \prod_{[\rho]} q^{|\Gamma / H| r(H, \rho)} \varphi\left(\left(M^{\langle\rho\rangle}\right)^{r(H, \rho)} ; \operatorname{Aut}_{\Gamma-G}\left(P_{\rho}\right) \imath \mathfrak{S}_{r(H, \rho)}\right) \\
& =\prod_{[H]} \prod_{[\rho]}\left[\sum_{r \geq 0} q^{|\Gamma / H| r} \varphi\left(\left(M^{\langle\rho\rangle}\right)^{r} ; \operatorname{Aut}_{\Gamma-G}\left(P_{\rho}\right) \curlywedge \mathfrak{S}_{r}\right)\right] .
\end{aligned}
$$

This completes the proof.

Recall that we have two kinds of orbifold Euler characteristics given in (1-3). We apply formula (5-3) with $\varphi(M ; G)=\chi_{\Gamma}^{\text {orb }}(M ; G)$, for various $\Gamma$ arising as fundamental groups of real 2-dimensional surfaces which are orientable or nonorientable, compact or non-compact. In the next section, we apply formula $(5-3)$ to $\chi_{\Gamma}(M ; G)$ [Theorem $\left.6-3\right]$. We will see that these formulae are closely related but very different.

Theorem 5-5 Let $G$ be a finite group, and let $M$ be a $G$-manifold. For any group $\Gamma$, we have

$$
\sum_{n \geq 0} q^{n} \chi_{\Gamma}^{\text {orb }}\left(M^{n} ; G \backslash \mathfrak{S}_{n}\right)=\exp \left[\sum_{r \geq 1} \frac{q^{r}}{r}\left\{\sum_{\substack{H \\|\Gamma / H|=r}} \chi_{H}^{\text {orb }}(M ; G)\right\}\right],
$$

where the second summation on the right hand side runs over all index $r$ subgroups of $\Gamma$.

Proof We apply formula (5-3) with $\varphi=\chi^{\text {orb }}$. By definition of $\chi^{\text {orb }}(M ; G)$ in (1-3), we have

$$
\begin{aligned}
\sum_{n \geq 0} q^{n} \chi_{\Gamma}^{\mathrm{orb}}\left(M^{n} ; G \imath \mathfrak{S}_{n}\right) & =\prod_{[H]} \prod_{[\rho]}\left[\sum_{r \geq 0} q^{|\Gamma / H| r} \chi^{\mathrm{orb}}\left(\left(M^{\langle\rho\rangle}\right)^{r} ; \operatorname{Aut}_{\Gamma-G}\left(P_{\rho}\right) \imath \mathfrak{S}_{r}\right)\right] \\
& =\prod_{[H]} \prod_{[\rho]}\left\{\sum_{r \geq 0} q^{|\Gamma / H| r} \frac{\chi\left(M^{\langle\rho\rangle}\right)^{r}}{\left|\operatorname{Aut}_{\Gamma-G}\left(P_{\rho}\right)\right|^{r} r !}\right\} \\
& =\prod_{[H]} \prod_{[\rho]} \exp \left[q^{|\Gamma / H|} \frac{\chi\left(M^{\langle\rho\rangle}\right)}{\left|\operatorname{Aut}_{\Gamma-G}\left(P_{\rho}\right)\right|}\right] \\
& =\exp \left[\sum_{r \geq 1} q^{r} \sum_{[H]} \sum_{[\rho / H \mid=r} \frac{\chi\left(M^{\langle\rho\rangle}\right)}{\left|\operatorname{Aut}_{\Gamma-G}\left(P_{\rho}\right)\right|}\right] .
\end{aligned}
$$

Algebraic \& $\mathcal{G}$ Geometric Topology, Volume 3 (2003) 
In the last formula, $[H]$ runs over all conjugacy classes of index $r$ subgroups of $\Gamma$, and for a given $[H],[\rho]$ runs over the set $\operatorname{Hom}(H, G) /\left(N_{\Gamma}(H) \times G\right)$.

Since the centralizer $C(\rho)$ is the isotropy subgroup of the conjugation action by $G$ on $\operatorname{Hom}(H, G)$ at $\rho$, we have $\#(\rho) \cdot|C(\rho)|=|G|$, where $(\rho)$ is the $G$ conjugacy class of $\rho$. Similarly, since $N_{\Gamma}^{\rho}(H) / H$ is the isotropy subgroup of the $N_{\Gamma}(H) / H$-action on the set of $G$-conjugacy classes $\operatorname{Hom}(H, G) / G$ at $(\rho)$, the length of the $N_{\Gamma}(H) / H$-orbit through $(\rho)$ is $\left|N_{\Gamma}(H) / N_{\Gamma}^{\rho}(H)\right|$. Now we continue our calculation. By Theorem 4-2, $\left|\operatorname{Aut}_{\Gamma-G}\left(P_{\rho}\right)\right|=|C(\rho)|\left|N_{\Gamma}^{\rho}(H) / H\right|$. Thus, for a fixed $[H]$, we have

$$
\begin{gathered}
\sum_{[\rho]} \frac{\chi\left(M^{\langle\rho\rangle}\right)}{\left|\operatorname{Aut}_{\Gamma-G}\left(P_{\rho}\right)\right|}=\sum_{[\rho]} \frac{\chi\left(M^{\langle\rho\rangle}\right)}{|C(\rho)|\left|N_{\Gamma}^{\rho}(H) / H\right|}=\sum_{(\rho)} \frac{\left|N_{\Gamma}^{\rho}(H) / H\right|}{\left|N_{\Gamma}(H) / H\right|} \frac{\chi\left(M^{\langle\rho\rangle}\right)}{|C(\rho)|\left|N_{\Gamma}^{\rho}(H) / H\right|} \\
=\frac{1}{\left|N_{\Gamma}(H) / H\right|} \sum_{(\rho)} \frac{\chi\left(M^{\langle\rho\rangle}\right)}{|C(\rho)|}=\frac{1}{\left|N_{\Gamma}(H) / H\right|} \sum_{\rho} \frac{\chi\left(M^{\langle\rho\rangle}\right)}{\#(\rho) \cdot|C(\rho)|} \\
=\frac{1}{\left|N_{\Gamma}(H) / H\right|} \frac{1}{|G|} \sum_{\rho} \chi\left(M^{\langle\rho\rangle}\right)=\frac{1}{\left|N_{\Gamma}(H) / H\right|} \chi_{H}^{\text {orb }}(M ; G) .
\end{gathered}
$$

Here, $[\rho]$ runs over the set $\operatorname{Hom}(H, G) /\left(N_{\Gamma}(H) \times H\right),(\rho)$ runs over the set of $G$ conjugacy classes $\operatorname{Hom}(H, G) / G$, and $\rho$ runs over the set of all homomorphisms $\operatorname{Hom}(H, G)$. The last equality is due to formula (2-4).

We convert the summation over the conjugacy classes $[H]$ of index $r$ subgroups to a summation over index $r$ subgroups $H$. Since there are $\left|\Gamma / N_{\Gamma}(H)\right|$ elements in the $\Gamma$-conjugacy class $[H]$,

$$
\begin{aligned}
\sum_{\substack{[H] \\
|\Gamma / H|=r}} \frac{1}{\left|N_{\Gamma}(H) / H\right|} \chi_{H}^{\mathrm{orb}}(M ; G) & =\sum_{\substack{H \\
|\Gamma / H|=r}} \frac{1}{\left|\Gamma / N_{\Gamma}(H)\right|\left|N_{\Gamma}(H) / H\right|} \chi_{H}^{\mathrm{orb}}(M ; G) \\
& =\sum_{\substack{H \\
|\Gamma / H|=r}} \frac{1}{|\Gamma / H|} \chi_{H}^{\mathrm{orb}}(M ; G)=\frac{1}{r} \sum_{\substack{H \\
|\Gamma / H|=r}} \chi_{H}^{\mathrm{orb}}(M ; G) .
\end{aligned}
$$

This completes the proof of formula (5-4).

The above formula implies that to calculate the orbifold Euler characteristic $\chi_{\Gamma}^{\text {orb }}\left(M^{n} ; G \imath \mathfrak{S}_{n}\right)$ of the $n$-fold symmetric product of an orbifold, we need to know $\chi_{H}^{\text {orb }}(M ; G)$ for every subgroup $H$ of $\Gamma$ whose index is at most $n$.

We specialize this formula. Letting $M=\mathrm{pt}$, we obtain the following combinatorial formula:

$$
\sum_{n \geq 0} q^{n} \frac{\left|\operatorname{Hom}\left(\Gamma, G \geq \mathfrak{S}_{n}\right)\right|}{|G|^{n} n !}=\exp \left[\sum_{r \geq 1} \frac{q^{r}}{r}\left\{\sum_{\substack{H \\|\Gamma / H|=r}} \frac{|\operatorname{Hom}(H, G)|}{|G|}\right\}\right] .
$$


This formula can be used to compute the number of homomorphisms into wreath products.

Next, we let $G$ be a trivial group in (5-4). Then we get

$$
\sum_{n \geq 0} q^{n} \chi_{\Gamma}^{\text {orb }}\left(M^{n} ; \mathfrak{S}_{n}\right)=\left(\exp \left[\sum_{r \geq 1} \frac{q^{r}}{r} j_{r}(\Gamma)\right]\right)^{\chi(M)},
$$

where $j_{r}(\Gamma)$ is the number of index $r$ subgroups of $\Gamma$. This formula shows that when $M$ varies, $\chi_{\Gamma}^{\mathrm{orb}}\left(M^{n} ; \mathfrak{S}_{n}\right)$ depends only on the Euler characteristic $\chi(M)$. Further specializing this formula to the case $M=\mathrm{pt}$, we get a well-known combinatorial formula [15, p.76]:

$$
\sum_{n \geq 0} q^{n} \frac{\left|\operatorname{Hom}\left(\Gamma, \mathfrak{S}_{n}\right)\right|}{n !}=\exp \left[\sum_{r \geq 1} \frac{q^{r}}{r} j_{r}(\Gamma)\right]
$$

A related combinatorial formula can be found in physics literature in the context of partition functions of permutation orbifolds [1].

We apply formula (5-4) to various groups $\Gamma$ to deduce numerous consequences.

\section{(i) Higher order ( $p$-primary) orbifold Euler characteristic}

These are orbifold Euler characteristics associated to $\Gamma=\mathbb{Z}^{d}$ or $\Gamma=\mathbb{Z}_{p}^{d}$, where $\mathbb{Z}_{p}$ is the ring of $p$-adic integers. The case $\Gamma=\mathbb{Z}^{d}$ corresponds to the case in which the manifold $\Sigma$ is a $d$-dimensional torus $T^{d}$ in our consideration of twisted sectors (2-11) and (2-12).

We need to know the number $j_{r}(\Gamma)$ of index $r$ subgroups for these groups $\Gamma$. This is well known, and we discussed these numbers in our previous paper [17, Lemma 4-4, Lemma 5-5].

Lemma 5-6 (1) For any $r \geq 1$ and $d \geq 1$, we have

$$
j_{r}\left(\mathbb{Z}^{d}\right)=\sum_{r_{1} r_{2} \cdots r_{d}=r} r_{2} r_{3}^{2} \cdots r_{d}^{d-1}, \quad \text { and } \quad j_{r}\left(\mathbb{Z}^{d}\right)=\sum_{m \mid r} m \cdot j_{m}\left(\mathbb{Z}^{d-1}\right) .
$$

(2) For any $r \geq 0$ and $d \geq 1$, we have

$$
j_{p^{r}}\left(\mathbb{Z}_{p}^{d}\right)=\sum_{\ell_{1}+\ell_{2}+\cdots+\ell_{d}=r} p^{\ell_{2}} p^{2 \ell_{3}} \cdots p^{(d-1) \ell_{d}}, \quad \text { and } \quad j_{p^{r}}\left(\mathbb{Z}_{p}^{d}\right)=\sum_{0 \leq \ell \leq r} p^{\ell} \cdot j_{p^{\ell}}\left(\mathbb{Z}_{p}^{d-1}\right) .
$$

One of the main results in [17] was the inductive calculation of the generating function of $\chi_{\Gamma}\left(M^{n} ; G \succ \mathfrak{S}_{n}\right)$ for $\Gamma=\mathbb{Z}^{d}, \mathbb{Z}_{p}^{d}$. (See Theorem 6-5.) Now with our general formula (5-4), the proof of the formula of generating functions for $\left\{\chi_{\Gamma}^{\text {orb }}\left(M^{n} ; G \imath \mathfrak{S}_{n}\right)\right\}_{n \geq 0}$ with $\Gamma=\mathbb{Z}^{d}, \mathbb{Z}_{p}^{d}$ is a straightforward corollary. Note that our proof of (5-4) was by a direct proof, not inductive proof. 
Theorem 5-7 Let $G$ be a finite group and let $M$ be a $G$-manifold. For $d \geq 1$,

$$
\begin{aligned}
& \sum_{n \geq 0} q^{n} \chi_{\mathbb{Z}^{d}}^{\mathrm{orb}}\left(M^{n} ; G \backslash \mathfrak{S}_{n}\right)=\left[\prod_{r \geq 1}\left(1-q^{r}\right)^{\left.-j_{r}\left(\mathbb{Z}^{d-1}\right)\right]^{\chi_{\mathbb{Z}^{d}}^{\mathrm{orb}}(M ; G)}}\right. \\
& \sum_{n \geq 0} q^{n} \chi_{\mathbb{Z}_{p}^{d}}^{\mathrm{orb}}\left(M^{n} ; G \backslash \mathfrak{S}_{n}\right)=\left[\prod_{r \geq 0}\left(1-q^{p^{r}}\right)^{-j_{p^{r}}\left(\mathbb{Z}_{p}^{d-1}\right)}\right]^{\chi_{\mathbb{Z}_{p}^{d}}^{\mathrm{orb}}(M ; G)} .
\end{aligned}
$$

Proof First note that for any $r \geq 1$, every index $r$ subgroup of $\mathbb{Z}^{d}$ is isomorphic to $\mathbb{Z}^{d}$, and any subgroup of $\mathbb{Z}_{p}^{d}$ has index a power of $p$ and any index $p^{r}$ subgroup of $\mathbb{Z}_{p}^{d}$ is again isomorphic to $\mathbb{Z}_{p}^{d}$. Thus, by (5-4),

$$
\begin{aligned}
\sum_{n \geq 0} q^{n} \chi_{\mathbb{Z}^{d}}^{\mathrm{orb}}\left(M^{n} ; G \imath \mathfrak{S}_{n}\right) & =\exp \left[\sum_{r \geq 1} \frac{q^{r}}{r} j_{r}\left(\mathbb{Z}^{d}\right) \chi_{\mathbb{Z}^{d}}^{\mathrm{orb}}(M ; G)\right] \\
& =\left(\exp \left[\sum_{r \geq 1} \frac{q^{r}}{r} j_{r}\left(\mathbb{Z}^{d}\right)\right]\right)^{\chi_{\mathbb{Z}^{d}}^{\text {orb }}(M ; G)} .
\end{aligned}
$$

For the remaining part, using the inductive formula in Lemma 5-6, we have

$$
\begin{aligned}
\exp \left[\sum_{r \geq 1} \frac{q^{r}}{r} j_{r}\left(\mathbb{Z}^{d}\right)\right] & =\exp \left[\sum_{r \geq 1} \frac{q^{r}}{r} \sum_{m \mid r} m \cdot j_{m}\left(\mathbb{Z}^{d-1}\right)\right] \\
= & \exp \left[\sum_{m \geq 1} j_{m}\left(\mathbb{Z}^{d-1}\right)\left(\sum_{\ell \geq 1} \frac{q^{m \ell}}{\ell}\right)\right]=\prod_{m \geq 1}\left(1-q^{m}\right)^{-j_{m}\left(\mathbb{Z}^{d-1}\right) .}
\end{aligned}
$$

Similarly for the $p$-adic case. This completes the proof.

The $d=0$ case in the above is elementary, and the formula was given in (2-10).

\section{(ii) Higher genus orbifold Euler characteristic (orientable case)}

Let $\Sigma_{g+1}$ be a real 2-dimensional closed orientable genus $g+1$ surface. Its fundamental group is the surface group $\Gamma_{g+1}$ described in (1-7). By covering space theory, conjugacy classes of index $r$ subgroups of $\Gamma_{g+1}$ are in $1: 1$ correspondence with the isomorphism classes of $r$-fold covering spaces over $\Sigma_{g+1}$. By Hurwitz's Theorem, or by a simple argument using Euler characteristic, we see that any such $r$-fold covering space of $\Sigma_{g+1}$ is a closed orientable surface of genus $r g+1$. Hence any index $r$ subgroup of $\Gamma_{g+1}$ is always isomorphic to $\Gamma_{r g+1}$, although they may sit inside of the group $\Gamma_{g+1}$ differently, and their conjugacy classes can be different. A direct application of Theorem 5-5 gives the next theorem. 
Theorem 5-8 Let $g \geq 0$. The generating function of the genus $g+1$ orbifold Euler characteristic of the $n$-th symmetric orbifold is given by

$$
\sum_{n \geq 0} q^{n} \chi_{\Gamma_{g+1}}^{\mathrm{orb}}\left(M^{n} ; G 乙 \mathfrak{S}_{n}\right)=\exp \left[\sum_{r \geq 1} q^{r}\left\{\frac{j_{r}\left(\Gamma_{g+1}\right)}{r} \chi_{\Gamma_{r g+1}}^{\mathrm{orb}}(M ; G)\right\}\right] .
$$

The above formula requires that we know the numbers $j_{r}\left(\Gamma_{g+1}\right)$ for $g \geq 0$. By letting $M=\mathrm{pt}$, we get the first combinatorial formula in (1-14). Further letting $G$ be trivial, we get

$$
\sum_{n \geq 0} q^{n} \frac{\operatorname{Hom}\left(\Gamma_{g+1}, \mathfrak{S}_{n}\right) \mid}{n !}=\exp \left[\sum_{r \geq 1} \frac{j_{r}\left(\Gamma_{g+1}\right)}{r} q^{r}\right] .
$$

The left hand side was given in (1-15) in terms of the character theory of $\mathfrak{S}_{n}$. Thus, $j_{r}\left(\Gamma_{g+1}\right)$ is, in principle, calculable.

\section{(iii) Orbifold Euler characteristic associated to free groups}

Let $\Gamma=F_{s+1}$ be a free group generated by $s+1$ elements, $s \geq 0$. Any index $r$ subgroup is isomorphic to $F_{r s+1}$, a free group with $r s+1$ generators. This can be most easily seen geometrically using covering spaces, as follows. Let $S$ be a torus with $s$ disjointly embedded discs removed with $s \geq 1$. Then $\pi_{1}(S) \cong F_{s+1}$. Any conjugacy class of an index $r$ subgroup corresponds to an isomorphism class of an $r$-fold covering space $\tilde{S}$ which is a torus with rs disjointly embedded discs removed. Hence $\pi_{1}(\tilde{S}) \cong F_{r s+1}$. Thus, any index $r$ subgroup of $F_{s+1}$ is again a free group isomorphic to $F_{r s+1}$. Note that any two index $r$ subgroups of $F_{s+1}$ are not necessarily conjugate to each other, although they are abstractly isomorphic. Again applying Theorem 5-5 directly, we get

Theorem 5-9 Let $s \geq 0$. Then

$$
\sum_{n \geq 0} q^{n} \chi_{F_{s+1}}^{\mathrm{orb}}\left(M^{n} ; G \geq \mathfrak{S}_{n}\right)=\exp \left[\sum_{r \geq 1}\left\{\frac{j_{r}\left(F_{s+1}\right)}{r} \chi_{F_{r s+1}}^{\mathrm{orb}}(M ; G)\right\} q^{r}\right] .
$$

Now we let $M=$ pt in the above formula. Since $\operatorname{Hom}\left(F_{r}, G\right) \cong G^{r}$, we have a following combinatorial formula

$$
\sum_{n \geq 0} q^{n}\left(|G|^{n} n !\right)^{s}=\exp \left[\sum_{r \geq 1} q^{r} \frac{j_{r}\left(F_{s+1}\right)}{r}|G|^{r s}\right] .
$$


How do we calculate $j_{r}\left(F_{s+1}\right)$, the number of index $r$ subgroups of a free group $F_{s+1}$ ? Letting $|G|=1$ in (5-12), we get a well known formula $[15$, p.76]

$$
\sum_{n \geq 0} q^{n}(n !)^{s}=\exp \left[\sum_{r \geq 1} q^{r} \frac{j_{r}\left(F_{s+1}\right)}{r}\right] .
$$

This formula determines $j_{r}\left(F_{s+1}\right)$ from known quantities. Actually, the formulae (5-12) and (5-13) are easily seen to be equivalent.

\section{(iv) Higher genus orbifold Euler characteristic (non-orientable case)}

Let $N_{h+2}$ be a closed non-orientable (real 2-dimensional) surface of genus $h+2$ for $h \geq 0$. The fundamental group $\Lambda_{h+2}=\pi_{1}\left(N_{h+2}\right)$ is described by (1-9). We apply Theorem 5-5 with $\Gamma=\Lambda_{h+2}$. Since $N_{1}=\mathbb{R} P^{2}$ has fundamental group $\mathbb{Z} / 2$, this case directly follows from (5-4). Here we only consider nonorientable surface groups of genus 2 or greater. First we need to know more about subgroups of $\Lambda_{h+2}$. We discuss this using covering spaces of $N_{h+2}$.

For $r \geq 1$, by examining Euler characteristic, we can easily see that any $r$ fold connected covering space of $N_{h+2}$ is either non-orientable $\left(\cong N_{r h+2}\right)$ or orientable $\left(\cong \Sigma_{\frac{r h}{2}+1}\right)$, and orientable covering spaces can occur only when $r$ is even. We call subgroups of $\Lambda_{h+2}$ corresponding to connected orientable covering spaces orientable subgroups. Let $j_{r}\left(\Lambda_{h+2}\right)^{+}$denote the number of index $r$ orientable subgroups of $\Lambda_{h+2}$. Similarly, let $j_{r}\left(\Lambda_{h+2}\right)^{-}$denote the number of index $r$ non-orientable subgroups. The surface $N_{h+2}$ has a unique orientable double cover $\Sigma_{h+1}$, and any orientable cover of $N_{h+2}$ is a cover of $\Sigma_{h+1}$. This means that $j_{\text {odd }}\left(\Lambda_{h+2}\right)^{+}=0$ and $j_{2 r}\left(\Lambda_{h+2}\right)^{+}=j_{r}\left(\Gamma_{h+1}\right)$ for all $r \geq 1$ and $h \geq 0$. Now we apply Theorem 5-5.

Theorem 5-10 Let $h \geq 0$. Then

$$
\begin{aligned}
\sum_{n \geq 0} q^{n} \chi_{\Lambda_{h+2}}^{\mathrm{orb}}\left(M^{n} ; G z \mathfrak{S}_{n}\right)=\exp \left[\sum_{r \geq 1} \frac{q^{r}}{r} j_{r}\left(\Lambda_{h+2}\right) \chi_{\Lambda_{h+2}}^{\text {orb }}(M ; G)\right. \\
\left.+\sum_{r \geq 1} \frac{q^{2 r}}{2 r} j_{r}\left(\Gamma_{h+1}\right)\left\{\chi_{\Gamma_{r h+1}}^{\text {orb }}(M ; G)-\chi_{\Lambda_{2 r h+2}}^{\text {orb }}(M ; G)\right\}\right] .
\end{aligned}
$$

Proof Theorem 5-5 implies that

$$
\begin{aligned}
& \sum_{n \geq 0} q^{n} \chi_{\Lambda_{h+2}}^{\text {orb }}\left(M^{n} ; G\left\langle\mathfrak{S}_{n}\right)\right. \\
& =\exp \left[\sum_{r \geq 1} \frac{q^{r}}{r}\left\{j_{r}\left(\Lambda_{h+2}\right)^{+} \chi_{\Gamma_{\frac{r h}{2}+1}^{\mathrm{orb}}}^{\text {orb }}(M ; G)+j_{r}\left(\Lambda_{h+2}\right)^{-} \chi_{\Lambda_{r h+2}}^{\mathrm{orb}}(M ; G)\right\}\right] .
\end{aligned}
$$

We only have to rewrite this formula using $j_{r}\left(\Lambda_{h+2}\right)^{-}=j_{r}\left(\Lambda_{h+2}\right)-j_{r}\left(\Lambda_{h+2}\right)^{+}$, and a fact that $j_{r}\left(\Lambda_{h+2}\right)^{+}=j_{\frac{r}{2}}\left(\Gamma_{h+1}\right)$ when $r$ is even and $j_{r}\left(\Lambda_{h+2}\right)^{+}=0$ when $r$ is odd. 
How do we calculate the number $j_{r}\left(\Lambda_{h+2}\right)$ ? We let $M=$ pt and let $G$ be a trivial group. Then the above formula reduces to

$$
\sum_{n \geq 0} q^{n} \frac{\left|\operatorname{Hom}\left(\Lambda_{h+2}, \mathfrak{S}_{n}\right)\right|}{n !}=\exp \left[\sum_{r \geq 1} \frac{q^{r}}{r} j_{r}\left(\Lambda_{h+2}\right)\right] .
$$

By using the character theory of $\mathfrak{S}_{n}$, one can show that for all $h \geq-1$,

$$
\frac{\left|\operatorname{Hom}\left(\Lambda_{h+2}, \mathfrak{S}_{n}\right)\right|}{n !}=\sum_{[V] \in \operatorname{Irred}\left(\mathfrak{S}_{n}\right)}\left(\frac{n !}{\operatorname{dim} V}\right)^{h} .
$$

The above two formulae determine $j_{r}\left(\Lambda_{h+2}\right)$. The formula (5-16) is a special case of Kerber-Wagner Theorem [9]. Their formula for a general finite group $G$, instead of $\mathfrak{S}_{n}$, is

$$
\frac{\left|\operatorname{Hom}\left(\Lambda_{h+2}, G\right)\right|}{|G|}=\sum_{\chi \in \operatorname{Irred}(G)}\left(\frac{|G|}{\operatorname{dim} \chi}\right)^{h} \varepsilon_{2}(\chi)^{h+2},
$$

where $\varepsilon_{2}(\chi)$ is the Schur indicator defined by $\varepsilon_{2}(\chi)=\sum_{g \in G} \chi\left(g^{2}\right) /|G|$. It is well known that $\varepsilon_{2}(\chi)=1$ if $\chi$ is of real type, $\varepsilon_{2}(\chi)=0$ if $\chi$ is of complex type, and $\varepsilon_{2}(\chi)=-1$ if $\chi$ is of quaternionic type. For details of this fact, see $\left[2\right.$, p.100]. It is well known that any representation of $\mathfrak{S}_{n}$ is of real type so that $\varepsilon(\chi)=1$ for any irreducible character $\chi$ of $\mathfrak{S}_{n}$.

Remark The formulae (5-10) and (1-15), and formulae (5-15) and (5-16) are very similar. By comparing these formulae it is immediate that $j_{r}\left(\Gamma_{h+1}\right)=$ $j_{r}\left(\Lambda_{2 h+2}\right)$ for all $r \geq 1$ and $h \geq 0$. However, this does not necessarily mean that $\chi_{\Gamma_{g+1}}^{\text {orb }}(M ; G)=\chi_{\Lambda_{2 g+2}}^{\text {orb }}(M ; G)$. In fact, they are in general different, although in some cases they coincide. See (5-22) for an example of such a case.

\section{(v) Klein bottle orbifold Euler characteristic}

We specialize our formula (5-14) to $h=0$ case. The corresponding nonorientable closed surface $N_{2}$ is the Klein bottle. By the remark above, we have $j_{r}\left(\Gamma_{1}\right)=j_{r}\left(\Lambda_{2}\right)$ for all $r \geq 1$. Since by Theorem $5-6, j_{r}\left(\Gamma_{1}\right)=j_{r}\left(\mathbb{Z}^{2}\right)=$ $\sum_{\ell \mid r} \ell=\sigma_{1}(r)$, the sum of positive divisors of $r$, the formula (5-14) reduces to

$$
\begin{aligned}
& \sum_{n \geq 0} q^{n} \chi_{\Lambda_{2}}^{\mathrm{orb}}\left(M^{n} ; G \geq \mathfrak{S}_{n}\right) \\
= & \exp \left[\sum_{r \geq 1} \frac{q^{r}}{r} \sigma_{1}(r) \chi_{\Lambda_{2}}^{\mathrm{orb}}(M ; G)+\sum_{r \geq 1} \frac{q^{2 r}}{2 r} \sigma_{1}(r)\left\{\chi_{\Gamma_{1}}^{\mathrm{orb}}(M ; G)-\chi_{\Lambda_{2}}^{\mathrm{orb}}(M ; G)\right\}\right] .
\end{aligned}
$$

Rewriting exponentials in terms of infinite products as in the proof of Theorem 5-7, we obtain 
Theorem 5-11 Let $G$ be a finite group and let $M$ be a $G$-manifold. Then (5-18)

$$
\sum_{n \geq 0} q^{n} \chi_{\Lambda_{2}}^{\mathrm{orb}}\left(M^{n} ; G \geq \mathfrak{S}_{n}\right)=\left[\prod_{r \geq 1}\left(1-q^{2 r}\right)\right]^{\frac{-1}{2} \chi_{\Gamma_{1}}^{\mathrm{orb}}(M ; G)}\left[\prod_{r \geq 1}\left(\frac{1+q^{r}}{1-q^{r}}\right)\right]^{\frac{1}{2} \chi_{\Lambda_{2}}^{\mathrm{orb}}(M ; G)} .
$$

Letting $M=\mathrm{pt}$, we obtain a combinatorial formula for the number of homomorphisms from the fundamental group of a Klein bottle into a wreath product:

$$
\begin{aligned}
& \sum_{n \geq 0} q^{n} \frac{\left.\mid \operatorname{Hom}\left(\Lambda_{2}, G\right) \mathfrak{S}_{n}\right) \mid}{|G|^{n} n !} \\
& \quad=\left[\prod_{\ell \geq 1}\left(1-q^{2 \ell}\right)\right]^{-\frac{1}{2}\left|\operatorname{Hom}\left(\Gamma_{1}, G\right)\right| /|G|}\left[\prod_{\ell \geq 1}\left(\frac{1+q^{\ell}}{1-q^{\ell}}\right)\right]^{\frac{1}{2}\left|\operatorname{Hom}\left(\Lambda_{2}, G\right)\right| /|G|} .
\end{aligned}
$$

Letting $G$ be trivial in (5-18), we get

$$
\sum_{n \geq 0} q^{n} \chi_{\Lambda_{2}}^{\mathrm{orb}}\left(M^{n} ; \mathfrak{S}_{n}\right)=\left[\prod_{\ell \geq 1}\left(1-q^{\ell}\right)\right]^{-\chi(M)} .
$$

On the other hand, when $d=2$ and $G$ is trivial, formula (5-8) reduces to

$$
\sum_{n \geq 0} q^{n} \chi_{\mathbb{Z}^{2}}^{\mathrm{orb}}\left(M^{n} ; \mathfrak{S}_{n}\right)=\left[\prod_{r \geq 1}\left(1-q^{r}\right)\right]^{-\chi(M)} .
$$

Comparing the above two formula, we see that $\chi_{\Gamma_{1}}^{\text {orb }}\left(M^{n} ; \mathfrak{S}_{n}\right)=\chi_{\Lambda_{2}}^{\text {orb }}\left(M^{n} ; \mathfrak{S}_{n}\right)$ for all $n \geq 0$, since $\Gamma_{1}=\mathbb{Z}^{2}$. This is no coincidence. In general, using (5-6) and a fact that $j_{r}\left(\Gamma_{g+1}\right)=j_{r}\left(\Lambda_{2 g+2}\right)$ for $g \geq 0, r \geq 1$ (see the remark at the end of (iv) in this section), we get

$$
\sum_{n \geq 0} q^{n} \chi_{\Gamma_{g+1}}^{\text {orb }}\left(M^{n} ; \mathfrak{S}_{n}\right)=\sum_{n \geq 0} q^{n} \chi_{\Lambda_{2 g+2}}^{\text {orb }}\left(M^{n} ; \mathfrak{S}_{n}\right), \quad g \geq 0
$$

\section{Orbifold invariants associated to $\Gamma$-sets and gen- erating functions: infinite product formulae}

In section 5 , we calculated the generating function $\sum_{n>0} q^{n} \varphi_{\Gamma}\left(M^{n} ; G \backslash \mathfrak{S}_{n}\right)$ for $\varphi(M ; G)=\chi^{\text {orb }}(M ; G)$, where $G$ is a finite group, $M$ is a $G$-manifold, and $\Gamma$ is any group. In this section, we investigate the generating function with $\varphi(M ; G)=\chi(M ; G)=\chi(M / G)$. The latter orbifold Euler characteristic is more closely tied to geometry of $G$-action than the former.

To describe the generating function $\sum_{n>0} q^{n} \chi_{\Gamma}\left(M^{n} ; G \backslash \mathfrak{S}_{n}\right)$, we need to introduce a notion of generalized orbifold invariants associated to transitive $\Gamma$-sets. 
Orbifold invariants associated to general $\Gamma$-sets will be described later in this section in (6-13). Let $H \subset \Gamma$ be a subgroup of finite index. Then the $\varphi$ orbifold invariant associated to the $\Gamma$-isomorphism class of a $\Gamma$-transitive set $\Gamma / H$ is defined by

$$
\begin{gathered}
\varphi_{[\Gamma / H]}(M ; G) \stackrel{\text { def }}{=} \sum \varphi\left(M^{\langle\rho\rangle} ; \operatorname{Aut}_{\Gamma-G}\left(P_{\rho}\right)\right), \\
{[\rho] \in \operatorname{Hom}(H, G) /\left(N_{\Gamma}(H) \times G\right)}
\end{gathered}
$$

where $\pi_{\rho}: P_{\rho}=\Gamma \times{ }_{\rho} G \rightarrow \Gamma / H$ is a $\Gamma$-irreducible $G$-bundle, and $\operatorname{Aut}_{\Gamma-G}\left(P_{\rho}\right)$ is the group of $\Gamma$-equivariant $G$-bundle automorphisms of $P_{\rho}$ described in Theorem 4-2 and Theorem 4-4.

When $H=\Gamma$, the conjugation on homomorphisms $\rho: \Gamma \rightarrow G$ by $N_{\Gamma}(\Gamma)=\Gamma$ is absorbed by the conjugation by $G$. Hence we have $\operatorname{Hom}(\Gamma, G) /\left(N_{\Gamma}(\Gamma) \times G\right)=$ $\operatorname{Hom}(\Gamma, G) / G$. Also in this case, $\operatorname{Aut}_{\Gamma-G}\left(P_{\rho}\right)$ reduces to $C_{G}(\rho)$ : see (4-2) for example. Hence $\varphi_{[\Gamma / \Gamma]}(M ; G)=\varphi_{\Gamma}(M ; G)$, recovering the $\Gamma$-extended orbifold invariant.

When $G$ is a trivial group $\{e\}, \rho$ is the trivial homomorphism and $\operatorname{Aut}_{\Gamma-G}\left(P_{\rho}\right)$ reduces to the group of $\Gamma$-automorphisms of the $\Gamma$-set $\Gamma / H$, that is $H \backslash N_{\Gamma}(H)$. Thus, $\varphi_{[\Gamma / H]}(M ;\{e\})=\varphi\left(M ; H \backslash N_{\Gamma}(H)\right)$, where $H \backslash N_{\Gamma}(H)$ acts trivially on $M$ by Proposition 5-3.

The above definition (6-1) may seem rather unusual, but it is the correct one. For example, the set over which $[\rho]$ runs has already appeared in the classification theorem of $\Gamma$-irreducible $G$-bundles in Theorem 3-6, and the above definition is closely related to this theorem.

To explain the origin of the definition, we consider the map

$$
\pi: \operatorname{Hom}\left(\Gamma, G_{n}\right) / G_{n} \rightarrow \operatorname{Hom}\left(\Gamma, \mathfrak{S}_{n}\right) / \mathfrak{S}_{n},
$$

induced by the projection map $G_{n} \rightarrow \mathfrak{S}_{n}$. Since the set $\operatorname{Hom}\left(\Gamma, \mathfrak{S}_{n}\right) / \mathfrak{S}_{n}$ can be regarded as the set of isomorphism classes of $\Gamma$-sets of order $n$, for any index $n$ subgroup $H$ of $\Gamma$, we may regard $[\Gamma / H] \in \operatorname{Hom}\left(\Gamma, \mathfrak{S}_{n}\right) / \mathfrak{S}_{n}$. The meaning of $\varphi_{[\Gamma / H]}(M ; G)$ is clarified by the next proposition.

Proposition 6-1 For any subgroup $H$ of index $n$ in $\Gamma$, we have

$$
\varphi_{[\Gamma / H]}(M ; G)=\sum_{[\theta] \in \pi^{-1}([\Gamma / H])} \varphi\left(\left(M^{n}\right)^{\langle\theta\rangle} ; C_{G_{n}}(\theta)\right),
$$

where $\pi: \operatorname{Hom}\left(\Gamma, G_{n}\right) / G_{n} \rightarrow \operatorname{Hom}\left(\Gamma, \mathfrak{S}_{n}\right) / \mathfrak{S}_{n}$ is the obvious map. 
Proof We rewrite the quantity in the right hand side in terms of $\Gamma$-equivariant $G$-bundles. By Theorem 3-1, any element in $\pi^{-1}([\Gamma / H])$ is an isomorphism class of a $\Gamma$-irreducible $G$-bundle over the $\Gamma$-set $\Gamma / H$. By the Classification Theorem 3-6 this set is in 1:1 correspondence with $\operatorname{Hom}(H, G) /\left(N_{\Gamma}(H) \times G\right)$, and the isomorphism class of the $\Gamma$-irreducible $G$-bundle corresponding to $[\theta] \in$ $\pi^{-1}([\Gamma / H])$ is the isomorphism class of $\pi_{\rho}: P_{\rho} \rightarrow \Gamma / H$, where $\rho: H \rightarrow G$ is constructed from $\theta$ (see the proof of Theorem 3-1), essentially by restriction to the isotropy subgroup $H \subset \Gamma$. Using Lemma 4-1 and Proposition 5-1, we have

$$
\sum_{[\theta] \in \pi^{-1}([\Gamma / H])} \varphi\left(\left(M^{n}\right)^{\langle\theta\rangle} ; C_{G_{n}}(\theta)\right)=\sum_{[\rho]} \varphi\left(M^{\langle\rho\rangle} ; \operatorname{Aut}_{\Gamma-G}\left(P_{\rho}\right)\right),
$$

where $[\rho]$ runs over the set $\operatorname{Hom}(H, G) /\left(N_{\Gamma}(H) \times G\right)$. Then formula (6-1) completes the proof.

Note that $\varphi_{[\Gamma / H]}(M ; G)$ is a partial sum of the summation defining $\varphi_{\Gamma}\left(M^{n} ; G_{n}\right)$ in (5-1). The situation here will be clarified in (6-14) after we define $\varphi_{[X]}(M ; G)$ for general $\Gamma$-set $X$.

We calculate an example of an orbifold invariant associated to a $\Gamma$-set. We denote an orbifold invariant $\chi_{\mathbb{Z}^{d}}(M ; G)$ by $\chi^{(d)}(M ; G)$. This is the $d$-th order orbifold Euler characteristic of $(M ; G)$ discussed in [17].

Lemma 6-2 Let $\Gamma$ be an abelian group, and let $d \geq 0$. Let $(M ; G)$ be as before. Then for any subgroup $H$ of finite index in $\Gamma$, we have

$$
\chi_{[\Gamma / H]}^{(d)}(M ; G)=|\Gamma / H|^{d} \cdot \chi_{H}^{(d)}(M ; G) .
$$

In particular, $\chi_{[\Gamma / H]}(M ; G)=\chi_{H}(M ; G)$, if $\Gamma$ is abelian.

Proof Let $\rho: H \rightarrow G$. To calculate $\chi_{[\Gamma / H]}^{(d)}(M ; G)$ defined in $(6-1)$, we need to understand the group $\operatorname{Aut}_{\Gamma-G}\left(P_{\rho}\right)$. The group $T_{\rho}$ given in (4-4) is, in our case, isomorphic to $\Gamma \times C_{G}(\rho)$ since $\Gamma$ is abelian. Then, by Theorem 4-4 we have $\operatorname{Aut}_{\Gamma-G}\left(P_{\rho}\right) \cong H \backslash T_{\rho} \cong \Gamma \times{ }_{\rho} C(\rho)$. From this, $\left|\operatorname{Aut}_{\Gamma-G}\left(P_{\rho}\right)\right|=|\Gamma / H||C(\rho)|$ and

$$
\chi_{[\Gamma / H]}^{(d)}(M ; G)=\sum_{[\rho]} \chi^{(d)}\left(M^{\langle\rho\rangle} ; \Gamma \times{ }_{\rho} C(\rho)\right)=\sum_{[\rho]} \frac{1}{|\Gamma / H||C(\rho)|} \sum_{\phi: \mathbb{Z}^{d+1} \rightarrow \Gamma \times{ }_{\rho} C(\rho)} \chi\left(\left(M^{\langle\rho\rangle}\right)^{\langle\phi\rangle}\right),
$$

where the last equality is due to $(2-4)$ and $(2-5)$, and $[\rho]$ runs over the set $\operatorname{Hom}(H, G) / G$, where $H$ is abelian. By Proposition 5-3, the image of the natural map $\iota: \Gamma \rightarrow \Gamma \times{ }_{\rho} C(\rho)$ acts trivially on $M^{\langle\rho\rangle}$. Let $\left\{\gamma_{i}\right\}_{i \in \Gamma / H} \subset \Gamma$ be the representatives of of the coset $\Gamma / H$. Then $\Gamma \times{ }_{\rho} C(\rho)=\coprod_{i \in \Gamma / H} \iota\left(\gamma_{i}\right) C(\rho)$. Note that two elements $\iota\left(\gamma_{i_{1}}\right) g_{1}$ and $\iota\left(\gamma_{i_{2}}\right) g_{2}$ in $\Gamma \times{ }_{\rho} C(\rho)$, where $g_{1}, g_{2} \in C(\rho)$, 
commute if and only if $g_{1}$ and $g_{2}$ commute in $C(\rho)$. Thus the above summation becomes

$$
\begin{aligned}
\sum_{[\rho]} \frac{1}{|\Gamma / H||C(\rho)|}|\Gamma / H|^{d+1} \sum_{\phi^{\prime}: \mathbb{Z}^{d+1} \longrightarrow C(\rho)} \chi\left(\left(M^{\langle\rho\rangle}\right)^{\left\langle\phi^{\prime}\right\rangle}\right) & =|\Gamma / H|^{d} \sum_{[\rho]} \chi^{(d)}\left(M^{\langle\rho\rangle} ; C(\rho)\right) \\
& =|\Gamma / H|^{d} \chi_{H}^{(d)}(M ; G) .
\end{aligned}
$$

Letting $d=0$, we obtain the last statement. This completes the proof.

Now we calculate the generating function $\sum_{n \geq 0} q^{n} \chi_{\Gamma}\left(M^{n} ; G \imath \mathfrak{S}_{n}\right)$.

Theorem 6-3 Let $G$ be a finite group and let $M$ be a $G$-manifold. For any group $\Gamma$, we have

$$
\begin{gathered}
\sum_{n \geq 0} q^{n} \chi_{\Gamma}\left(M^{n} ; G \geq \mathfrak{S}_{n}\right)=\prod_{r \geq 1}\left(1-q^{r}\right)^{-\sum_{[H]_{r}} \chi_{[\Gamma / H]}(M ; G)}, \\
\text { where } \quad \chi_{[\Gamma / H]}(M ; G)=\sum_{[\rho] \in \operatorname{Hom}(H, G) /\left(N_{\Gamma}(H) \times G\right)} \chi\left(M^{\langle\rho\rangle} / \operatorname{Aut}_{\Gamma-G}\left(P_{\rho}\right)\right) .
\end{gathered}
$$

Here in the summation in the right hand side of the first identity, $[H]_{r}$ means that $H$ runs over all the conjugacy classes of index $r$ subgroups of $\Gamma$, and the action of $\operatorname{Aut}_{\Gamma-G}\left(P_{\rho}\right)$ on $M^{\langle\rho\rangle}$ in the second formula is described in Proposition $5-3$.

When $\Gamma$ is an abelian group,

$$
\sum_{n \geq 0} q^{n} \chi_{\Gamma}\left(M^{n} ; G \imath \mathfrak{S}_{n}\right)=\prod_{r \geq 1}\left(1-q^{r}\right)^{-\sum_{H_{r}} \chi_{H}(M ; G)}
$$

where in the summation in the right hand side, $H_{r}$ means that $H$ runs over all index $r$ subgroups in $\Gamma$.

Proof For (6-4), by Proposition 5-4 and Macdonald's formula (2-9), we have

$$
\begin{gathered}
\sum_{n \geq 0} q^{n} \chi_{\Gamma}\left(M^{n} ; G \succ \mathfrak{S}_{n}\right)=\prod_{[H]} \prod_{[\rho]}\left[\sum_{r \geq 0} q^{|\Gamma / H| r} \chi\left(\left(M^{\langle\rho\rangle}\right)^{r} ; \operatorname{Aut}_{\Gamma-G}\left(P_{\rho}\right)\left\langle\mathfrak{S}_{r}\right)\right]\right. \\
=\prod_{[H]} \prod_{[\rho]}\left[\sum_{r \geq 0} q^{|\Gamma / H| r} \chi\left(S P^{r}\left(M^{\langle\rho\rangle} / \operatorname{Aut}_{\Gamma-G}\left(P_{\rho}\right)\right)\right)\right. \\
=\prod_{[H][\rho]}\left(1-q^{|\Gamma / H|}\right)^{-\chi\left(M^{\langle\rho\rangle} / \operatorname{Aut}_{\Gamma-G}\left(P_{\rho}\right)\right)}=\prod_{r \geq 1}\left(1-q^{r}\right)^{-\sum_{[H]} \sum_{r} \chi \rho\left(M^{\langle\rho\rangle} ; \operatorname{Aut}_{\Gamma-G}\left(P_{\rho}\right)\right)},
\end{gathered}
$$

where in the last summation $[H]_{r}$ runs over all the conjugacy classes of finite index in $\Gamma$, and $[\rho]$ runs over the set $\operatorname{Hom}(H, G) /\left(N_{\Gamma}(H) \times G\right)$. Notice that the summation over $[\rho]$ in the exponent in the last line exactly gives $\chi_{[\Gamma / H]}(M ; G)$. 
When $\Gamma$ is an abelian group, the formula (6-5) follows from (6-4) in view of Lemma 6-2 with $d=0$. Since $\Gamma$ is abelian, each subgroup $H$ is its own conjugacy class $[H]$. This completes the proof.

Note that in the corresponding formula for $\chi_{\Gamma}^{\text {orb }}$ given in (5-4), the summation there was over subgroups $H$ rather than conjugacy classes $[H]$ used in (6-4).

Remark From the above proof, one might think that the concept of orbifold invariant associated to a finite $\Gamma$-set is just a convenient symbol representing a complicated summation given in the right hand side of (6-1). However, as we will see in section 7 , this concept has a definitely natural geometric origin analogous to twisted sectors discussed in section 2 .

Letting $M=$ pt or letting $G$ be trivial, we immediately have the following corollary.

Corollary 6-4 (1) For any group $\Gamma$ and any finite group $G$, we have

$$
\sum_{n \geq 0} q^{n}\left|\operatorname{Hom}\left(\Gamma, G_{n}\right) / G_{n}\right|=\prod_{r \geq 1}\left(1-q^{r}\right)^{-\sum_{[H]_{r}}\left|\operatorname{Hom}(H, G) /\left(N_{\Gamma}(H) \times G\right)\right|} .
$$

Furthermore, when $\Gamma$ is abelian, we may omit the term $N_{\Gamma}(H)$ from the above expression.

(2) For any $\Gamma$ and any manifold $M$, we have

$$
\sum_{n \geq 0} q^{n} \chi_{\Gamma}\left(M^{n} ; \mathfrak{S}_{n}\right)=\left[\prod_{r \geq 1}\left(1-q^{r}\right)^{-u_{r}(\Gamma)}\right]^{\chi(M)},
$$

where $u_{r}(\Gamma)$ is the number of conjugacy classes of index $r$ subgroups of $\Gamma$.

The proof for (1) is straightforward. For (2), all we need to note is that when the group $G$ is trivial, $(6-1)$ gives that $\chi_{[\Gamma / H]}(M ;\{e\})=\chi\left(M ; H \backslash N_{\Gamma}(H)\right)=\chi(M)$, since the group $H \backslash N_{\Gamma}(H)$ acts trivially on $M$ by Proposition 5-3.

If we further specialize (6-7) to the case $M=$ pt or if we let $G=\{e\}$ in (6-6), then we obtain the following well known formula [15, p.76]:

$$
\sum_{n \geq 0} q^{n}\left|\operatorname{Hom}\left(\Gamma, \mathfrak{S}_{n}\right) / \mathfrak{S}_{n}\right|=\prod_{r \geq 1}\left(1-q^{r}\right)^{-u_{r}(\Gamma)} .
$$

Recall that $\left|\operatorname{Hom}\left(\Gamma, \mathfrak{S}_{n}\right) / \mathfrak{S}_{n}\right|=\left|\operatorname{Hom}\left(\Gamma \times \mathbb{Z}, \mathfrak{S}_{n}\right)\right| / n$ !. The above formula can be used to calculate the number of conjugacy classes of a given index in the orientable surface group $\Gamma_{g+1}$ of genus $g+1$, in the free group $F_{s+1}$ on $s+1$ generators, and in the non-orientable surface group $\Lambda_{h+2}$ of genus $h+2$. We discuss details in section 8 , where a general formula to calculate $u_{r}(\Gamma)$ is proved. We apply Theorem 6-3 to various groups $\Gamma$ to obtain various results. 
(i) Higher order ( $p$-primary) orbifold Euler characteristic of symmetric orbifolds

Suppose $\Gamma=\mathbb{Z}^{d}$ or $\mathbb{Z}_{p}^{d}$ for $d \geq 0$. Then the corresponding $\Gamma$-extended orbifold Euler characteristic is what we call higher order ( $p$-primary) orbifold Euler characteristic denoted by $\chi^{(d)}(M ; G)$ or $\chi_{p}^{(d)}(M ; G)$, respectively, in [17]. Since any index $r$ subgroup $H$ of $\mathbb{Z}^{d}$ is isomorphic to $\mathbb{Z}^{d}$ for any $r \geq 1$, we get the following theorem as a direct consequence of Theorem 6-3 for abelian $\Gamma$. Similarly for the case $\Gamma=\mathbb{Z}_{p}^{d}$. In [17], the same theorem was proved by induction.

Theorem 6-5 For any $d \geq 0$, we have

$$
\begin{aligned}
& \sum_{n \geq 0} q^{n} \chi^{(d)}\left(M^{n} ; G \smile \mathfrak{S}_{n}\right)=\left[\prod_{r \geq 1}\left(1-q^{r}\right)^{\left.-j_{r}\left(\mathbb{Z}^{d}\right)\right]^{\chi^{(d)}(M ; G)}}\right. \\
& \sum_{n \geq 0} q^{n} \chi_{p}^{(d)}\left(M^{n} ; G \smile \mathfrak{S}_{n}\right)=\left[\prod_{r \geq 0}\left(1-q^{p^{r}}\right)^{-j_{p^{r}}\left(\mathbb{Z}_{p}^{d}\right)}\right]^{\chi_{p}^{(d)}(M ; G)} .
\end{aligned}
$$

Proof All we need to note is that since $\Gamma=\mathbb{Z}^{d}$ is abelian, we can apply formula (6-5), and that the number $u_{r}\left(\mathbb{Z}^{d}\right)$ of conjugacy classes of index $r$ subgroups of $\mathbb{Z}^{d}$ is the same as the number $j_{r}\left(\mathbb{Z}^{d}\right)$ of index $r$ subgroups. The corresponding statement for $\mathbb{Z}_{p}^{d}$ is also valid.

\section{(ii) Higher genus orbifold Euler characteristic of symmetric orbifolds}

A direct application of Theorem 6-3 to the case $\Gamma=\Gamma_{g+1}$ for $g \geq 0$ gives the following.

Theorem 6-6 Let $g \geq 0$. Then, with $[H]_{r}$ as before,

$$
\sum_{n \geq 0} q^{n} \chi_{\Gamma_{g+1}}\left(M^{n} ; G \geq \mathfrak{S}_{n}\right)=\prod_{r \geq 1}\left(1-q^{r}\right)^{-\sum_{[H]_{r}} \chi_{\left[\Gamma_{g+1} / H\right]}(M ; G)}
$$

Although all index $r$ subgroups of $\Gamma_{g+1}$ are isomorphic to each other $\left(\cong \Gamma_{r g+1}\right)$, they may not be conjugate to each other. This is why we cannot be any more concrete than the above expression. In the formula (6-6) with $\Gamma=\Gamma_{g+1}$, normalizers of index $r$ subgroups appear. The size of the normalizer $N_{\Gamma_{g+1}}(H)$ can be different for different $[H]$ of the same index. Geometrically, the group $N_{\Gamma}(H) / H$ is the group of deck transformations of the covering space $\Sigma_{r g+1} \rightarrow$ $\Sigma_{g+1}$ corresponding to the conjugacy class of the index $r$ subgroup $H$. Thus $\chi_{\left[\Gamma_{g+1} / H\right]}(M ; G)$ really depends on the conjugacy class $[H]$. 
The summation in the exponent of the right hand side of (6-9) can be thought of as a summation over all isomorphism classes of index $r$ covering spaces over $\Sigma_{g+1}$. See section 7 for details.

A similar formula is valid when $\Gamma$ is the fundamental group $\Lambda_{h+2}$ of nonorientable genus $h+2$ closed surface, or a free group $F_{s+1}$ on $s+1$ generators. We omit their explicit expressions here. Again, any further analysis requires information on normalizers of subgroups.

(iii) Higher order higher genus orbifold Euler characteristic of symmetric orbifolds

We combine the previous two cases and consider the generating function for the invariant $\chi_{\Gamma}^{(d)}(M ; G)=\chi_{\mathbb{Z}^{d} \times \Gamma}(M ; G)$. This equality is due to $(2-2)$. We also consider the case for $\chi_{p, \Gamma}^{(d)}$.

Theorem 6-7 For any $d \geq 0$ and any $\Gamma$, with $[H]_{\ell}$ as before, we have

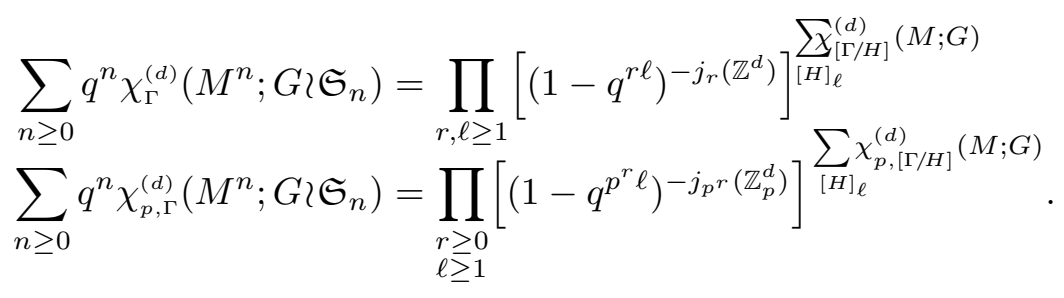

Proof By Proposition 5-4 and Theorem 6-5, we have

$$
\begin{aligned}
& \sum_{n \geq 0} q^{n} \chi_{\Gamma}^{(d)}\left(M^{n} ; G \imath \mathfrak{S}_{n}\right)=\prod_{[H]} \prod_{[\rho]}\left[\sum_{r \geq 0} q^{|\Gamma / H| r} \chi^{(d)}\left(\left(M^{\langle\rho\rangle}\right)^{r} ; \operatorname{Aut}_{\Gamma-G}\left(P_{\rho}\right) \curlywedge \mathfrak{S}_{r}\right)\right] \\
& =\prod_{[H]} \prod_{[\rho]}\left[\prod_{r \geq 1}\left(1-q^{r|\Gamma / H|}\right)^{\left.-j_{r}\left(\mathbb{Z}^{d}\right)\right]^{(d)}\left(M^{\langle\rho\rangle} ; \operatorname{Aut}_{\Gamma-G}\left(P_{\rho}\right)\right)}\right. \\
& =\prod_{[H]}\left[\prod_{r \geq 1}\left(1-q^{r|\Gamma / H|}\right)^{\left.-j_{r}\left(\mathbb{Z}^{d}\right)\right]_{(d)}^{\chi_{[\Gamma / H]}^{(d)}(M ; G)}}\right. \\
& =\prod_{\ell \geq 1}\left[\prod_{r \geq 1}\left(1-q^{r \ell}\right)^{-j_{r}\left(\mathbb{Z}^{d}\right)}\right]_{[H]_{\ell}}^{\sum_{[\Gamma / H]}^{(d)}(M ; G)},
\end{aligned}
$$

here, for the third equality, we summed over $[\rho] \in \operatorname{Hom}(H, G) /\left(N_{\Gamma}(H) \times G\right)$, and for the fourth equality, we first summed over conjugacy classes of index $\ell$ subgroups of $\Gamma$. The case for $\chi_{p, \Gamma}^{(d)}$ is similar. This completes the proof. 
The generating functions of two types of orbifold Euler characteristics of symmetric orbifolds given in Theorem 5-5 and Theorem 6-3 seem very different. But by (1-5), these two orbifold Euler characteristics are related by a simple formula $\chi_{\Gamma \times \mathbb{Z}}^{\mathrm{orb}}(M ; G)=\chi_{\Gamma}(M ; G)$. Thus we expect to get a nontrivial identity by comparing these two formulae of generating functions. We compare these two formulae for $M=\mathrm{pt}$. The corresponding formulae are (5-5) and (6-6).

Now, applying (2-6) to the wreath product $G_{n}$, we obtain

$$
\frac{\left|\operatorname{Hom}\left(\Gamma \times \mathbb{Z}, G_{n}\right)\right|}{\left|G_{n}\right|}=\left|\operatorname{Hom}\left(\Gamma, G_{n}\right) / G_{n}\right| .
$$

This is the link between (5-5) and (6-6). By directly comparing the right hand sides of corresponding formulae, we obtain the identity:

$$
\exp \left[\sum_{r \geq 1} \frac{q^{r}}{r}\left\{\sum_{\substack{K \\[\Gamma \times \mathbb{Z}: K]=r}} \frac{|\operatorname{Hom}(K, G)|}{|G|}\right\}\right]=\prod_{r \geq 1}\left(1-q^{r}\right)^{-\sum_{[H]_{r}}\left|\operatorname{Hom}(H, G) /\left(N_{\Gamma}(H) \times G\right)\right|} .
$$

Expanding this identity, we see that the following proposition must hold. Although the identity (6-11) is its proof, we also give a group theoretic proof to show what kind of group theoretic ingredients are involved.

Proposition 6-8 Let $G$ be a finite group and let $\Gamma$ be an arbitrary group. For any $r \geq 1$, we have

$$
\sum_{[\Gamma \times \mathbb{Z} ; K]=r} \frac{|\operatorname{Hom}(K, G)|}{|G|}=\sum_{\ell \mid r} \ell\left\{\sum_{\substack{[H] \\|\Gamma / H|=\ell}}\left|\operatorname{Hom}(H, G) /\left(N_{\Gamma}(H) \times G\right)\right|\right\} .
$$

When $G$ is trivial, this formula reduces to a well-known formula $j_{r}(\Gamma \times \mathbb{Z})=$ $\sum_{\ell \mid r} \ell \cdot u_{\ell}(\Gamma)$.

Proof Let $\ell \mid r$. For an index $\ell$ subgroup $H \subset \Gamma$ and $\bar{z} \in N_{\Gamma}(H) / H$, let

$$
K_{(H, \bar{z})}=\left\{(w, r a / \ell) \in N_{\Gamma}(H) \times \mathbb{Z} \mid \bar{w}=\bar{z}^{a} \text { in } N_{\Gamma}(H) / H\right\} \subset \Gamma \times \mathbb{Z} .
$$

Then $K_{(H, \bar{z})}$ is a subgroup of index $r$ in $\Gamma \times \mathbb{Z}$, and any index $r$ subgroup of $\Gamma \times \mathbb{Z}$ is of this form [16, p.140]. Thus, index $r$ subgroups of $\Gamma \times \mathbb{Z}$ are parametrized by the pair $(H, \bar{z})$, where the index of $H$ divides $r$ and $\bar{z}$ is an arbitrary element in $N_{\Gamma}(H) / H$. Let $z \in N_{\Gamma}(H)$ be any element whose reduction $\bmod H$ is $\bar{z}$. Then any element in $K_{(H, \bar{z})}$ is of the form $(z, r / \ell)^{a} \cdot(h, 0) \in N_{\Gamma}(H) \times \mathbb{Z}$ for some unique $a \in \mathbb{Z}$ and $h \in H$. Now let $\phi: K_{(H, \bar{z})} \rightarrow G$ be a homomorphism. Let $\rho=\left.\phi\right|_{H}: H \rightarrow G$ be its restriction. Let $\phi((z, r / \ell))=g \in G$. Since we have $(z, r / \ell)(h, 0)(z, r / \ell)^{-1}=\left(z h z^{-1}, 0\right)$ in $K_{(H, \bar{z})}$, we have $g \rho(h) g^{-1}=\rho\left(z h z^{-1}\right)$ in $G$. This means that $\rho^{z}$, defined by $\rho^{z}(h)=\rho\left(z h z^{-1}\right)$ for $h \in H$, and $\rho$ are 
$G$-conjugate, which we denote by $\rho^{z} \sim \rho$. Conversely, given any $\rho: H \rightarrow G$ and any $g \in G$ satisfying a relation $g \rho(h) g^{-1}=\rho^{z}(h)$ for any $h \in H$, we can define a homomorphism $\phi: K_{(H, \bar{z})} \rightarrow G$ by $\phi\left(\left(z^{a} h, r a / \ell\right)\right)=g^{a} \rho(h)$. Since for a given $\rho: H \rightarrow G$ such that $\rho^{z} \sim \rho$, there are $|C(\rho)|$ choices of $g \in G$ satisfying $g \rho g^{-1}=\rho^{z}$, we see that $\left|\operatorname{Hom}\left(K_{(H, \bar{z})}, G\right)\right|=\sum_{\rho}|C(\rho)|$, where the summation runs over all $\rho: H \rightarrow G$ such that $\rho^{z} \sim \rho$. Hence,

$$
\begin{aligned}
& \sum_{\bar{z} \in N_{\Gamma}(H) / H} \frac{\left|\operatorname{Hom}\left(K_{(H \bar{z})}, G\right)\right|}{|G|}=\sum_{\bar{z} \in N_{\Gamma}(H) / H} \sum_{\substack{\rho: H \rightarrow G \\
\rho^{z} \sim \rho}} \frac{|C(\rho)|}{|G|}=\sum_{\rho: H \rightarrow G} \frac{|C(\rho)|}{|G|} \sum_{\substack{z \in N_{\Gamma}(H) / H \\
\rho^{z} \sim \rho}} 1 \\
&=\sum_{\rho: H \rightarrow G} \frac{|C(\rho)|}{|G|}\left|N_{\Gamma}^{\rho}(H) / H\right|=\sum_{\rho: H \rightarrow G} \frac{1}{\#(\rho)}\left|N_{\Gamma}^{\rho}(H) / H\right|=\sum_{(\rho) \in \operatorname{Hom}(H, G) / G}\left|N_{\Gamma}^{\rho}(H) / H\right| .
\end{aligned}
$$

Here $(\rho)$ denotes the $G$-conjugacy class of $\rho$. The third equality above is by definition of $N_{\Gamma}^{\rho}(H)$ in (4-1). On the other hand, $\left|\operatorname{Hom}(H, G) /\left(N_{\Gamma}(H) \times G\right)\right|$ is equal to the number of orbits under the $N_{\Gamma}(H)$-action on $\operatorname{Hom}(H, G) / G$. At $(\rho) \in \operatorname{Hom}(H, G) / G$, the isotropy subgroup of $N_{\Gamma}(H)$-action is $N_{\Gamma}^{\rho}(H)$. Thus,

$$
\begin{gathered}
\left|\operatorname{Hom}(H, G) /\left(N_{\Gamma}(H) \times G\right)\right|=\sum_{(\rho) \in \operatorname{Hom}(H, G) / G} 1 / \mid(\rho) \text {-orbit }\left|=\sum_{(\rho) \in \operatorname{Hom}(H, G) / G}\right| N_{\Gamma}^{\rho}(H) / N_{\Gamma}(H) \mid \\
=\left(1 /\left|N_{\Gamma}(H) / H\right|\right) \sum_{(\rho) \in \operatorname{Hom}(H, G) / G}\left|N_{\Gamma}^{\rho}(H) / H\right|=\frac{\#[H]}{\ell_{\bar{z} \in N_{\Gamma}(H) / H}} \frac{\left|\operatorname{Hom}\left(K_{(H, \bar{z})}, G\right)\right|}{|G|},
\end{gathered}
$$

where $\mid(\rho)$-orbit| denotes the length of the $N_{\Gamma}(H)$-orbit in $\operatorname{Hom}(H, G) / G$ through $(\rho)$. The last equality is by the above calculation. Since index $r$ subgroups of $\Gamma \times \mathbb{Z}$ are parametrized by pairs $(H, \bar{z})$, where $H$ is a subgroup of $\Gamma$ whose index divides $r$ and $\bar{z} \in N_{\Gamma}(H) / H$, we have

$$
\begin{gathered}
\sum_{[\Gamma \times \mathbb{Z}: K]=r} \frac{|\operatorname{Hom}(K, G)|}{|G|}=\sum_{\ell \mid r} \sum_{H_{\ell}} \sum_{\bar{z} \in N_{\Gamma}(H) / H} \frac{\left|\operatorname{Hom}\left(K_{(H, \bar{z})}, G\right)\right|}{|G|} \\
=\sum_{\ell \mid r} \sum_{H_{\ell}} \frac{\ell}{\#[H]}\left|\operatorname{Hom}(H, G) /\left(N_{\Gamma}(H) \times G\right)\right| \\
=\sum_{\ell \mid r} \sum_{[H]_{\ell}} \ell\left|\operatorname{Hom}(H, G) /\left(N_{\Gamma}(H) \times G\right)\right| .
\end{gathered}
$$

This completes the proof.

From the proof, it is clear that $K_{(H, \bar{z})}$ is a semi-direct product $H \rtimes \mathbb{Z}$, where $\mathbb{Z} \subset K_{(H, \bar{z})}$ is a subgroup generated by the element $(z, r / \ell) \in K_{(H, \bar{z})}$. 
We next describe orbifold invariants associated to general $\Gamma$-sets. Let $X$ be any $\Gamma$-set of order $n$. Then for any given multiplicative invariant $\varphi(M ; G)$, we define the orbifold invariant associated to the $\Gamma$-isomorphism class $[X]$ by (6-13)

$$
\varphi_{[X]}(M ; G) \stackrel{\text { def }}{=} \sum_{[P \longrightarrow X]} \varphi\left(\mathcal{S}\left[P \times_{G} M\right]^{\Gamma} ; \operatorname{Aut}_{\Gamma-G}(P)\right) \cong \sum_{[\theta] \in \pi^{-1}([X])} \varphi\left(\left(M^{n}\right)^{\langle\theta\rangle} ; C_{G_{n}}(\theta)\right),
$$

where the first summation is over all isomorphism classes of $\Gamma$-equivariant $G$ principal bundles $P$ over $X$, and $\pi: \operatorname{Hom}\left(\Gamma, G_{n}\right) / G_{n} \rightarrow \operatorname{Hom}\left(\Gamma, \mathfrak{S}_{n}\right) / \mathfrak{S}_{n}$ is as before. When $X$ is an empty set $\emptyset$, we let $\varphi_{\emptyset}(M ; G)=1$. The second isomorphism is due to Theorem 3-1, Lemma 4-1 and Proposition 5-1. The details of the proof are similar to the proof of Proposition 6-1. We could define $\varphi_{[\Gamma / H]}(M ; G)$ by the above conceptual formula instead of $(6-1)$, but $(6-1)$ is more explicit. The geometric definition (6-13) in terms of $\Gamma$-equivariant $G$-principal bundles has an advantage in its simplicity.

In view of Theorem 3-1 and (6-13), generating functions for $\varphi_{\Gamma}$ and $\varphi_{[X]}$ are related by

$$
\sum_{n \geq 0} q^{n} \varphi_{\Gamma}\left(M^{n} ; G \succ \mathfrak{S}_{n}\right)=\sum_{[X]} q^{|X|} \varphi_{[X]}(M ; G),
$$

where on the right hand side, $[X]$ runs over the set of all the isomorphism classes of finite $\Gamma$-sets.

Next we discuss multiplicativity of $\varphi_{[X]}(M ; G)$ with respect to $[X]$. It is not true that we have $\varphi_{[X]}(M ; G)=\varphi_{\left[X_{1}\right]}(M ; G) \cdot \varphi_{\left[X_{2}\right]}(M ; G)$ whenever $X=X_{1} \amalg X_{2}$. However, when $X_{1}$ and $X_{2}$ are "prime" to each other, namely when $X_{1}$ and $X_{2}$ do not contain the same $\Gamma$-transitive sets in common in their decomposition into $\Gamma$-orbits, the above multiplicativity is valid.

Proposition 6-9 Let $\varphi(M ; G)$ be a multiplicative orbifold invariant for $(M ; G)$. Let $\Gamma$ be a group. For any finite $\Gamma$-set $X$, let $X=\coprod_{[H]} r(H) \cdot \Gamma / H$ be its decomposition into $\Gamma$-orbits, where $r(H)$ is the number of $\Gamma$-orbits which are isomorphic to $\Gamma / H$. Then

$$
\varphi_{[X]}(M ; G)=\prod_{[H]} \varphi_{r(H)[\Gamma / H]}(M ; G) .
$$

Consequently, the generating function (6-14) decomposes into a product

$$
\sum_{n \geq 0} q^{n} \varphi_{\Gamma}\left(M^{n} ; G \imath \mathfrak{S}_{n}\right)=\prod_{[H]}\left\{\sum_{r \geq 0} q^{|\Gamma / H| r} \varphi_{r[\Gamma / H]}(M ; G)\right\} .
$$


Furthermore, the generating function of $\varphi_{r[\Gamma / H]}(M ; G)$ for $r \geq 0$ is given by

$$
\sum_{r \geq 0} q^{r} \varphi_{r[\Gamma / H]}(M ; G)=\prod_{[\rho]}\left\{\sum_{r \geq 0} q^{r} \varphi\left(\left(M^{\langle\rho\rangle}\right)^{r} ; \operatorname{Aut}_{\Gamma-G}\left(P_{\rho}\right)\left\langle\mathfrak{S}_{r}\right)\right\},\right.
$$

where $[\rho]$ runs over the set $\operatorname{Hom}(H, G) /\left(N_{\Gamma}(H) \times G\right)$.

Proof For any $\Gamma$-equivariant $G$-bundle $P$ over $X$, let $P_{H}$ be the part of $P$ above $r(H)[\Gamma / H]$. Then, using the multiplicativity of $\varphi(\cdot ; \cdot)$, we have

$$
\begin{aligned}
\varphi_{[X]}(M ; G) & =\sum_{[P \longrightarrow X]} \varphi\left(\mathcal{S}\left[P \times_{G} M\right]^{\Gamma} ; \operatorname{Aut}_{\Gamma-G}(P)\right) \\
& =\sum_{[P \longrightarrow X][H]} \varphi\left(\mathcal{S}\left[P_{H} \times_{G} M\right]^{\Gamma} ; \operatorname{Aut}_{\Gamma-G}\left(P_{H}\right)\right) \\
& =\prod_{[H]} \sum_{\left[P_{H} \rightarrow r(H) \cdot \Gamma / H\right]} \varphi\left(\mathcal{S}\left[P_{H} \times_{G} M\right]^{\Gamma} ; \operatorname{Aut}_{\Gamma-G}\left(P_{H}\right)\right)=\prod_{[H]} \varphi_{r(H)[\Gamma / H]}(M ; G),
\end{aligned}
$$

where in the fourth expression, $\left[P_{H}\right]$ rums over the set of all isomorphism classes of $\Gamma-G$ bundles over the $\Gamma$-set $r(H)[\Gamma / H]$. The formula (6-16) follows from this.

The proof of (6-17) is analogous to the argument in the proof of Proposition 5 -4, and goes as follows. For any $\Gamma$-equivariant $G$-bundle over $\amalg^{r} \Gamma / H$, let $r_{\rho}$ be the number of $\Gamma$-irreducible $G$-bundle $P_{\rho} \rightarrow \Gamma / H$ appearing in the irreducible decomposition of $P$, where $[\rho] \in \operatorname{Hom}(H, G) /\left(N_{\Gamma}(H) \times G\right)$. Then, decomposing $\Gamma$-equivariant $G$-bundles $P \longrightarrow r \cdot \Gamma / H$ according to $[\rho]$, we have

$$
\begin{aligned}
\sum_{r \geq 0} q^{r} \varphi_{r[\Gamma / H]}(M ; G) & \left.=\sum_{r \geq 0} q^{r} \sum_{[\rho]} \prod_{r_{\rho}=r} \varphi\left(\left(M^{\langle\rho\rangle}\right)\right)^{r_{\rho}} ; \operatorname{Aut}_{\Gamma-G}\left(P_{\rho}\right) \imath \mathfrak{S}_{r_{\rho}}\right) \\
& =\sum_{r_{\rho} \geq 0} \prod_{[\rho]} q^{r_{\rho}} \varphi\left(\left(M^{\langle\rho\rangle}\right)^{r_{\rho}} ; \operatorname{Aut}_{\Gamma-G}\left(P_{\rho}\right)\left\langle\mathfrak{S}_{r_{\rho}}\right)\right. \\
& =\prod_{[\rho]}\left\{\sum_{r \geq 0} q^{r} \varphi\left(\left(M^{\langle\rho\rangle}\right)^{r} ; \operatorname{Aut}_{\Gamma-G}\left(P_{\rho}\right)\left\langle\mathfrak{S}_{r}\right)\right\} .\right.
\end{aligned}
$$

This completes the proof.

Note that formulae (6-14), (6-16), and (6-17) essentially give a proof of Proposition 5-4, from the point of view of $\Gamma$-sets.

Next we examine the case in which $G$ is the trivial group. We have already discussed this case for a transitive $\Gamma$-set $X$. The proof of the following lemma is straightforward. 
Lemma 6-10 With the same notations as above, suppose $G=\{e\}$, the trivial group. Then

$$
\varphi_{[X]}(M ;\{e\})=\prod_{[H]} \varphi\left(M^{r(H)} ;\left(H \backslash N_{\Gamma}(H)\right) \imath \mathfrak{S}_{r(H)}\right),
$$

where $H \backslash N_{\Gamma}(H)$ acts trivially on $M$. For the case of the orbifold Euler characteristic, we have

$$
\chi_{[X]}(M ;\{e\})=\prod_{[H]} \chi\left(S P^{r(H)}(M)\right) .
$$

In particular, if $X$ is a transitive $\Gamma$-set, then $\chi_{[X]}(M ;\{e\})=\chi(M)$.

\section{$7 \quad$ Orbifold invariants associated to covering spaces}

Let $\Sigma$ be a connected manifold and let $M$ be a $G$-manifold where $G$ is a finite group. In section 2 , we considered a twisted space $\mathbb{L}_{\Sigma}(M ; G)$ defined by

$$
\mathbb{L}_{\Sigma}(M ; G)=\coprod_{[P]}\left[\operatorname{Map}_{G}(P, M) / \operatorname{Aut}_{G}(P)\right],
$$

where $[P]$ runs over the set of isomorphism classes of $G$-principal bundles over $\Sigma$, and $\operatorname{Aut}_{G}(P)$ is the group of $G$-bundle automorphisms inducing the identity map on the base $\Sigma$. Let $\Gamma=\pi_{1}(\Sigma)$. We defined the orbifold invariant associated to a group $\Gamma$ in (2-1) as the orbifold invariant of the subset of $\mathbb{L}_{\Sigma}(M ; G)$ consisting of locally constant $G$-equivariant maps described in (2-14):

$$
\varphi_{\Gamma}(M ; G)=\varphi_{\text {orb }}\left(\mathbb{L}_{\Sigma}(M ; G)_{\text {l.c. }}\right)=\sum_{[\phi] \in \operatorname{Hom}(\Gamma, G) / G} \varphi\left(M^{\langle\phi\rangle} ; C(\phi)\right),
$$

where l.c. stands for "locally constant."

We generalize the above construction of the twisted space $\mathbb{L}_{\Sigma}(M ; G)$ associated to $\Sigma$ to twisted spaces associated to finite covering spaces of $\Sigma$. Let $\pi_{\Sigma^{\prime} \Sigma \Sigma}$ : $\Sigma^{\prime} \rightarrow \Sigma$ be a (not necessarily connected) finite covering space of $\Sigma$. The idea of constructing twisted spaces in this new context is the same as before: we consider liftings of maps $\operatorname{Map}\left(\Sigma^{\prime}, M / G\right)$ to maps from $G$-principal bundles $P$ over $\Sigma^{\prime}$ to $M$. So let $\pi: P \rightarrow \Sigma^{\prime}$ be a $G$-principal bundle over $\Sigma^{\prime}$ and consider the set $\operatorname{Map}_{G}(P, M)$ of $G$-equivariant maps from $P$ to $M$. Now the equivalence relation we introduce among these maps is different from the one in section 2. Given two $G$-maps $\gamma_{1}: P_{1} \rightarrow M$ and $\gamma_{2}: P_{2} \rightarrow M, \gamma_{1}$ and $\gamma_{2}$ are said to be equivalent in the present context if there exists a $G$-bundle isomorphism $\alpha: P_{1} \rightarrow P_{2}$ inducing a deck transformation of $\Sigma^{\prime}$ over $\Sigma$ such 
that $\gamma_{2} \circ \alpha=\gamma_{1}$. We call such $\alpha$ a $G$-bundle isomorphism over a covering space $\Sigma^{\prime} / \Sigma$.

Let $\operatorname{Aut}_{G}(P)_{\Sigma^{\prime} / \Sigma}$ be the group of $G$-automorphisms of $P$ over the covering space $\Sigma^{\prime} / \Sigma$. The set of equivalence classes denoted by $\mathbb{L}_{\Sigma^{\prime} \Sigma \Sigma}(M ; G)$ is our new twisted space associated to the covering space $\Sigma^{\prime} / \Sigma$. Thus,

$$
\mathbb{L}_{\Sigma^{\prime} / \Sigma}(M ; G)=\coprod_{\left[P \rightarrow \Sigma^{\prime} / \Sigma\right]}\left[\operatorname{Map}_{G}(P, M) / \operatorname{Aut}_{G}(P)_{\Sigma^{\prime} / \Sigma}\right] .
$$

Here the disjoint union runs over the set of all $G$-bundle isomorphism classes over the covering space $\Sigma^{\prime} / \Sigma$ in the above sense. We consider the subset of locally constant $G$-equivariant maps:

$$
\mathbb{L}_{\Sigma^{\prime} / \Sigma}(M ; G)_{\text {l.c. }}=\coprod_{\left[P \longrightarrow \Sigma^{\prime} / \Sigma\right]}\left[\operatorname{Map}_{G}(P, M)_{\text {l.c. }} / \operatorname{Aut}_{G}(P)_{\Sigma^{\prime} / \Sigma}\right] .
$$

Here note that the group $\operatorname{Aut}_{G}(P)_{\Sigma^{\prime} / \Sigma}$ still acts on the $\operatorname{space}^{\operatorname{Map}_{G}}(P, M)_{\text {l.c. }}$. of locally constant $G$-equivariant maps. By definition, the orbifold invariant associated to a finite covering $\Sigma^{\prime} \rightarrow \Sigma$ is the $\varphi$-invariant of $\mathbb{L}_{\Sigma^{\prime} / \Sigma}(M ; G)_{\text {l.c. }}$, viewed equivariantly rather than as a quotient:

$$
\varphi_{\left[\Sigma^{\prime} / \Sigma\right]}(M ; G)=\varphi_{\text {orb }}\left(\mathbb{L}_{\Sigma^{\prime} / \Sigma}(M ; G)_{\text {l.c. }}\right) \stackrel{\text { def }}{=} \sum_{\left[P \longrightarrow \Sigma^{\prime} / \Sigma\right]} \varphi\left(\operatorname{Map}_{G}(P, M)_{\text {l.c. }} ; \operatorname{Aut}_{G}(P)_{\Sigma^{\prime} / \Sigma}\right) .
$$

We analyze quantities appearing in the above formula to prove the following theorem, which is the main result of this section.

Theorem 7-1 Let $\pi_{\Sigma^{\prime} \Sigma \Sigma}: \Sigma^{\prime} \rightarrow \Sigma$ be a finite (not necessarily connected) covering space over a connected manifold $\Sigma$ with a base point $x_{0}$. Let $\Gamma=\pi_{1}\left(\Sigma, x_{0}\right)$. Let $X=\Sigma_{x_{0}}^{\prime}$ be the fibre over $x_{0} \in \Sigma$, and let $[X]$ be its isomorphism class as a $\Gamma$-set. Then, the orbifold invariant associated to the covering $\Sigma / \Sigma$ defined in (7-5) is equal to the orbifold invariant associated to the isomorphism class of the $\Gamma$-set $[X]$ defined in (6-13):

$$
\varphi_{\left[\Sigma^{\prime} / \Sigma\right]}(M ; G)=\varphi_{[X]}(M ; G) .
$$

The invariant $\varphi_{\left[\Sigma^{\prime} \Sigma\right]}(M ; G)$ is multiplicative in the following sense. Let $\pi_{1}$ : $\Sigma_{1}^{\prime} \rightarrow \Sigma$ and $\pi_{2}: \Sigma_{2}^{\prime} \rightarrow \Sigma$ be two coverings over $\Sigma$. Suppose $\Sigma_{1}^{\prime}$ and $\Sigma_{2}^{\prime}$ do not contain any isomorphic connected coverings in common. Then

$$
\varphi_{\left[\left(\Sigma_{1}^{\prime} \cup \Sigma_{2}^{\prime}\right) / \Sigma\right]}(M ; G)=\varphi_{\left[\Sigma_{1}^{\prime} / \Sigma\right]}(M ; G) \varphi_{\left[\Sigma_{2}^{\prime} / \Sigma\right]}(M ; G) .
$$


Since any finite $\Gamma$-set $X$ can be a fibre of some finite covering space $\Sigma^{\prime}=$ $\widetilde{\Sigma} \times_{\Gamma} X \rightarrow \Sigma$, where $\widetilde{\Sigma}$ is the universal covering space of $\Sigma$, the identity (7-6) gives a geometric meaning of orbifold invariants associated to $\Gamma$-sets in terms of covering spaces. The formula (7-7) corresponds to the formula (6-15) for orbifold invariants associated to $\Gamma$-sets.

To prove Theorem 7-1, we first recall some basic facts on (universal) covering spaces. Let $\widetilde{\pi}: \widetilde{\Sigma} \rightarrow \Sigma$ be the universal covering space of $\Sigma$, and let $x_{0} \in \Sigma$ be a base point. Let $\Gamma=\pi_{1}\left(\Sigma, x_{0}\right)$ be the fundamental group of $\Sigma$. One convenient description of $\tilde{\Sigma}$ is the following one:

$(7-8)$

$\widetilde{\Sigma}=\left\{(x,[\gamma]) \mid x \in \Sigma\right.$ and $[\gamma]$ is the homotopy class of a path $\gamma$ from $x$ to $\left.x_{0}\right\}$.

We can introduce a suitable topology on $\widetilde{\Sigma}$ so that the projection map $\widetilde{\Sigma} \rightarrow \Sigma$ is a covering map. In this description, the point $(x,[\gamma]) \in \widetilde{\Sigma}$ is the end point of the lift in $\widetilde{\Sigma}$ of $\gamma^{-1}$ starting at the natural base point $y_{0}=\left(x_{0},\left[c_{x_{0}}\right]\right)$ of $\widetilde{\Sigma}$, where $c_{x_{0}}$ is the constant path at $x_{0} \in \Sigma$. The fundamental group $\Gamma$ acts from the right on $\widetilde{\Sigma}$ as deck transformations by

$$
\begin{gathered}
\widetilde{\Sigma} \times \Gamma \longrightarrow \widetilde{\Sigma} \\
((x,[\gamma]),[\eta]) \mapsto(x,[\gamma][\eta]),
\end{gathered}
$$

where $[\eta] \in \Gamma$. This action is well defined because $\gamma$ is a path from $x$ to the base point $x_{0}$ and $\eta$ is a loop at $x_{0}$. Here, we regard $\widetilde{\Sigma}$ as a right $\Gamma$-principal bundle. This is the reason why we let $\Gamma$ act on $\Sigma$ on the right. On the other hand, the group $\Gamma$ also acts on the fibre $\widetilde{F}_{x_{0}}=\widetilde{\pi}^{-1}\left(x_{0}\right)$ from the left by

$$
\begin{gathered}
\Gamma \times \widetilde{F}_{x_{0}} \longrightarrow \widetilde{F}_{x_{0}} \\
\left([\eta],\left(x_{0},[\gamma]\right)\right) \mapsto\left(x_{0},[\eta][\gamma]\right) .
\end{gathered}
$$

Again, this is well defined because the path $\gamma$ is now a loop based at $x_{0}$. Note that for a given $y=\left(x_{0},[\gamma]\right)$, the result of the action of $[\eta]$ on $y,[\eta] \cdot y$, is the end point of the lift in $\widetilde{\Sigma}$ of $\eta^{-1}$ starting at $y$. Obviously, the left action of $\Gamma$ on $\widetilde{F}_{x_{0}}$, and the right action of $\Gamma$ as deck transformations restricted to $\widetilde{F}_{x_{0}}$, commute. In some literature, the action of $\Gamma$ on the fibre is defined from the right. We decided to use the above left action in this paper to make it explicit that the action of $\Gamma$ on the fibre commutes with its action on $\widetilde{\Sigma}$ as deck transformations.

The story is similar for any connected covering space $\pi_{\Sigma^{\prime} \Sigma \Sigma}: \Sigma^{\prime} \rightarrow \Sigma$. Any such $\Sigma^{\prime}$ is isomorphic to a covering space of the form $\widetilde{\Sigma} / H$ for some subgroup $H \subset \Gamma$, determined up to conjugacy, and can be described as $\Sigma^{\prime}=\{(x,[\gamma] H)\}$ where $x \in \Sigma$ and $[\gamma]$ is the homotopy class of paths from $x$ to the base point 
$x_{0}$. The point $y_{0}=\left(x_{0}, H\right) \in \Sigma^{\prime}$ can be used as the natural base point of $\Sigma^{\prime} \cong \widetilde{\Sigma} / H$. The group of deck transformations for the covering space $\Sigma^{\prime} \rightarrow \Sigma$ given by $G\left(\Sigma^{\prime} / \Sigma\right) \cong N_{\Gamma}(H) / H$ acts on $\Sigma^{\prime}$ from the right, and $\Gamma$ acts on the fibre $F_{x_{0}}=\pi_{\Sigma^{\prime} \Sigma}^{-1}\left(x_{0}\right)$ over $x_{0}$ from the left in the same way as for $\widetilde{\Sigma}$ :

$$
\begin{aligned}
& \Gamma \times F_{x_{0}} \longrightarrow F_{x_{0}} \quad \Sigma^{\prime} \times\left(N_{\Gamma}(H) / H\right) \longrightarrow \Sigma^{\prime} \\
& \left([\eta],\left(x_{0},[\gamma] H\right)\right) \mapsto\left(x_{0},[\eta][\gamma] H\right), \quad((x,[\gamma] H),[\eta] H) \mapsto(x,[\gamma][\eta] H) .
\end{aligned}
$$

For any $y \in F_{x_{0}}$, the point $[\eta] \cdot y \in F_{x_{0}}$ is obtained as the end point of the lift in $\Sigma^{\prime}$ of $\eta^{-1}$ starting at $y \in F_{x_{0}}$. Similarly, given $y=(x,[\gamma] H) \in \Sigma^{\prime}$, the result of the action of the deck transformation by $[\eta] H$ on $y$ is the point $y \cdot[\eta] H$ obtained as the end point of the lift in $\Sigma^{\prime}$ of $\gamma^{-1}$ starting at $[\eta] \cdot y_{0}=\left(x_{0},[\eta] H\right)$.

If $\Sigma^{\prime}$ is not connected, the above facts apply to each connected component. Note that the intersection of a connected component of $\Sigma^{\prime}$ with the fibre $F_{x_{0}}$ is a single $\Gamma$-orbit.

Next we study basic properties of $G$-principal bundles over covering spaces. Let $\pi^{\prime}: P \rightarrow \Sigma^{\prime}$ be a $G$-principal bundle over a not necessarily connected covering space $\Sigma^{\prime}$ over $\Sigma$. Let $\pi=\pi_{\Sigma^{\prime} / \Sigma} \circ \pi^{\prime}: P \rightarrow \Sigma$. This is a covering space over $\Sigma$ on which $G$ acts as a group of deck transformations. Let $P_{x_{0}}=\pi^{-1}\left(x_{0}\right)$ and $\Sigma_{x_{0}}^{\prime}=\pi_{\Sigma^{\prime} \Sigma}^{-1}\left(x_{0}\right)$ be fibres over $x_{0}$. Then $P_{x_{0}}$ has the structure of a $\Gamma$ equivariant $G$-principal bundle over $\Sigma_{x_{0}}^{\prime}$. It is easy to see that the bundle $P$ is completely determined by its fibre $P_{x_{0}}$, in the following way.

Proposition 7-2 Let $\pi^{\prime}: P \rightarrow \Sigma^{\prime}$ be a $G$-principal bundle over a covering space $\pi_{\Sigma^{\prime} \Sigma}: \Sigma^{\prime} \rightarrow \Sigma$, where $\Sigma$ is a connected manifold with a base point $x_{0}$. The the fibre $P_{x_{0}}$ has the structure of a $\Gamma$-equivariant $G$-principal bundle over $\Sigma_{x_{0}}^{\prime}$, and $\pi^{\prime}: P \rightarrow \Sigma$ is determined by the $\Gamma$-equivariant $G$-bundle structure of the fibre $P_{x_{0}} \rightarrow \Sigma_{x_{0}}^{\prime}$. That is, we have the following commutative diagram:

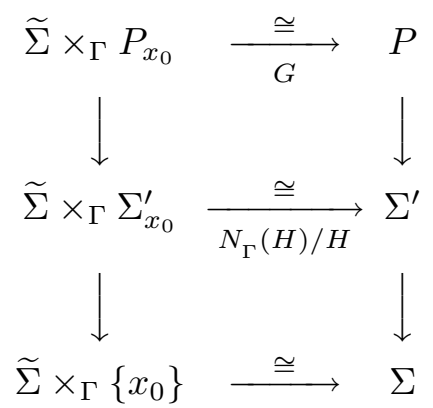

Here the top horizontal map is a $G$-equivariant map sending $[(x,[\gamma]), p]$ to $p^{\prime} \in P$ over $x \in \Sigma$ obtained as the end point of the lift in $P$ of $\gamma^{-1}$ starting at $p \in P$. The middle horizontal map is $N_{\Gamma}(H) / H$-equivariant map sending $\left[(x,[\gamma]),\left(x_{0},[\eta] H\right)\right]$ to $(x,[\gamma][\eta] H)$. 
Proof The proof is routine. So we only give a brief proof for the top horizontal map. First we check that this correspondence is well defined. For any $[\eta] \in \Gamma$, we consider two pairs $((x,[\gamma][\eta]), p)$ and $((x,[\gamma]),[\eta] p)$ in $\widetilde{\Sigma} \times P_{x_{0}}$, and compare the corresponding points in $P$ given by the procedure described above. The point $p^{\prime}$ corresponding to the first pair is the end point of the lift in $P$ of $(\gamma \eta)^{-1}=\eta^{-1} \gamma^{-1}$ starting at $p$. Since $[\eta] \cdot p$ is the end point of the lift in $P$ of $\eta^{-1}$ starting at $p$, the point $p^{\prime}$ is the same as the end point $p^{\prime \prime}$ of the lift in $P$ of $\gamma^{-1}$ starting at $[\eta] \cdot p$. But $p^{\prime \prime}$ is also the point corresponding to the second pair above. This proves that the top horizontal correspondence is well defined.

For $G$-equivariance, the point corresponding to $[(x,[\gamma]), p]$ is the end point $p_{1}$ of the lift $\widetilde{\gamma}_{1}^{-1}$ in $P$ of $\gamma^{-1}$ starting at $p$. Now the point corresponding to $[(x,[\gamma]), p g]$ for $g \in G$ is the end point $p_{2}$ of the lift $\widetilde{\gamma}_{2}^{-1}$ of $\gamma^{-1}$ starting at $p g$. Since both paths $\widetilde{\gamma}_{1}^{-1} \cdot g$ and $\widetilde{\gamma}_{2}^{-1}$ are lifts of $\gamma^{-1}$ and have the same starting point, they must coincide. Hence their end points are the same points and we have $p_{2}=p_{1} g$. Hence this correspondence is right $G$-equivariant.

To see surjectivity, for any point $p^{\prime} \in P$ over $x \in \Sigma$, we choose any path $\widetilde{\gamma}$ in $P$ from $p^{\prime}$ to a point $p$ in the fibre $P_{x_{0}}$ over $x_{0}$. Let $\gamma$ be the path in $\Sigma$ obtained by projecting $\widetilde{\gamma}$ into $\Sigma$. So $\gamma$ is a path from $x$ to $x_{0}$. Then $p^{\prime} \in P$ is the point corresponding to $[(x,[\gamma]), p] \in \widetilde{\Sigma} \times_{\Gamma} P_{x_{0}}$.

For injectivity, suppose two points $\left[\left(x_{1},\left[\gamma_{1}\right]\right), p_{1}\right]$ and $\left[\left(x_{2},\left[\gamma_{2}\right]\right), p_{2}\right]$ in $\widetilde{\Sigma} \times_{\Gamma} P_{x_{0}}$ correspond to the same point $p^{\prime}$ in $P$. We first note that $x_{1}=\pi\left(p^{\prime}\right)=x_{2}$. Next, let $\widetilde{\gamma_{1}}$ and $\widetilde{\gamma_{2}}$ be lifts in $P$ of $\gamma_{1}, \gamma_{2}$ starting at the same point $p^{\prime}$ and ending at $p_{1}$ and $p_{2}$ in $P_{x_{0}}$, respectively. Then a path $\widetilde{\gamma}_{2}^{-1} \cdot \widetilde{\gamma}_{1}$ is the lift of a loop $\eta=\gamma_{2}^{-1} \gamma_{1}$ based at $x_{0}$. So $\left[\gamma_{1}\right]=\left[\gamma_{2}\right][\eta]$ and $p_{2}=[\eta] \cdot p_{1}$. This implies that $\left[\left(x_{1},\left[\gamma_{1}\right]\right), p_{1}\right]=\left[\left(x_{1},\left[\gamma_{2}\right][\eta]\right), p_{1}\right]=\left[\left(x_{2},\left[\gamma_{2}\right]\right),[\eta] \cdot p_{1}\right]=\left[\left(x_{2},\left[\gamma_{2}\right]\right), p_{2}\right]$. This proves injectivity.

The proof for the middle horizontal map is similar.

This proposition reduces the comparison of $G$-bundles over covering spaces to the comparison of fibres as $\Gamma$-equivariant $G$-bundles.

Proposition 7-3 Let $\Sigma^{\prime} \rightarrow \Sigma$ be a covering space over a connected manifold $\Sigma$ with a base point $x_{0}$. Two $G$-principal bundles $\pi_{1}^{\prime}: P_{1} \rightarrow \Sigma^{\prime}$ and $\pi_{2}^{\prime}: P_{2} \rightarrow \Sigma^{\prime}$ are isomorphic as $G$-bundles over the covering space $\Sigma^{\prime} / \Sigma$ by an isomorphism $\alpha: P_{1} \stackrel{\cong}{\longrightarrow} P_{2}$ inducing a deck transformation of $\Sigma^{\prime} / \Sigma$ if and only if its restriction to fibres $\alpha_{x_{0}}: P_{1, x_{0}} \stackrel{\cong}{\cong} P_{2, x_{0}}$ is an isomorphism as $\Gamma$-equivariant $G$-bundles 
over the $\Gamma$-set $\Sigma_{x_{0}}^{\prime}$. Thus, we have the following bijective correspondence:

$$
\begin{aligned}
& \left\{\begin{array}{l}
\text { Isomorphism classes of } G \text {-principal bundles } \\
\pi: P \rightarrow \Sigma^{\prime} \text { over a covering space } \Sigma^{\prime} \Sigma \Sigma
\end{array}\right\} \\
& \qquad \stackrel{1: 1}{\stackrel{\text { onto }}{\longrightarrow}}\left\{\begin{array}{l}
\text { Isomorphism classes of } \Gamma \text {-equivariant } \\
G \text {-principal bundles } P_{x_{0}} \rightarrow \Sigma_{x_{0}}^{\prime}
\end{array}\right\} .
\end{aligned}
$$

In terms of isomorphisms, we have the following correspondences:

$$
\begin{aligned}
& \operatorname{Iso}_{G}\left(P_{1}, P_{2}\right)_{\Sigma^{\prime} / \Sigma} \underset{\text { onto }}{\stackrel{1: 1}{\leftrightarrows}} \operatorname{Iso}_{\Gamma-G}\left(P_{1, x_{0}}, P_{2, x_{0}}\right), \\
& \operatorname{Aut}_{G}(P)_{\Sigma / \Sigma} \underset{\text { onto }}{\stackrel{1: 1}{\longrightarrow}} \operatorname{Aut}_{\Gamma-G}\left(P_{x_{0}}\right)
\end{aligned}
$$

Proof The correspondence (7-15) is a special case of (7-14) with $P_{1}=P_{2}$. The correspondence (7-13) follows immediately from (7-14) by checking whether the sets are empty or not. Thus it suffices to prove bijectivity of the correspondence in (7-14).

Let $\alpha: P_{1} \rightarrow P_{2}$ be a $G$-bundle isomorphism over the covering space $\Sigma^{\prime} / \Sigma$. Since $\alpha$ is an isomorphism of covering spaces over $\Sigma$, it induces a $\Gamma=\pi_{1}(\Sigma)$ isomorphism of fibres $P_{1, x_{0}}$ and $P_{2, x_{0}}$. Since $\alpha$ is also a fibre preserving $G$ equivariant map, the restriction $\alpha_{x_{0}}: P_{1, x_{0}} \rightarrow P_{2, x_{0}}$ is a $\Gamma$-equivariant $G$ bundle isomorphism. This defines the correspondence in (7-14) in one direction.

Conversely, suppose we are given two $G$-principal bundles $\pi_{i}: P_{i} \rightarrow \Sigma^{\prime}$ for $i=1,2$ together with a $\Gamma$-equivariant $G$-bundle isomorphism $\phi: P_{1, x_{0}} \cong P_{2, x_{0}}$ between the fibres over $x_{0}$. By Proposition 7-2, we have the following $G$-bundle isomorphisms over the covering space $\Sigma / / \Sigma$ between $P_{1}$ and $P_{2}$ :

$$
P_{1} \cong \widetilde{\Sigma} \times_{\Gamma} P_{1, x_{0}} \underset{1 \times_{\Gamma} \phi}{\cong} \widetilde{\Sigma} \times_{\Gamma} P_{2, x_{0}} \stackrel{\cong}{\cong} P_{2} .
$$

This defines the correspondence in the other direction in (7-14). It is straightforward to check that the above two correspondences are inverse to each other. This completes the proof.

Combining with Classification Theorem 3-6, Theorem 4-2, and Theorem 4-4, we obtain the following corollary for the case of a connected covering space $\Sigma^{\prime} \rightarrow \Sigma$. 
Corollary 7-4 Let $\pi_{\Sigma^{\prime} / \Sigma}: \Sigma^{\prime} \rightarrow \Sigma$ be a connected finite covering space corresponding to the conjugacy class $[H]$ in $\Gamma=\pi_{1}\left(\Sigma, x_{0}\right)$, where $x_{0} \in \Sigma$ is a base point.

(1) We have the following bijective correspondence:

(7-16)

$\left\{\begin{array}{l}\text { Isomorphism classes of } G \text {-principal bundles } \\ \pi^{\prime}: P \rightarrow \Sigma^{\prime} \text { over the covering space } \Sigma^{\prime} / \Sigma\end{array}\right\} \underset{\text { onto }}{\stackrel{1: 1}{\longrightarrow}} \operatorname{Hom}(H, G) /\left(N_{\Gamma}(H) \times G\right)$.

(2) Let $\pi^{\prime}: P \rightarrow \Sigma^{\prime}$ be a $G$-principal bundle over a connected covering space $\Sigma / \Sigma$ whose holonomy homomorphism is given by a homomorphism $\rho: H \rightarrow G$, unique up to conjugation by $G$. Then the group of $G$-automorphisms of $P$ over the covering space $\Sigma^{\prime} / \Sigma$ (inducing deck transformations on $\Sigma^{\prime}$ ) is given by

$$
\operatorname{Aut}_{G}(P)_{\Sigma / \Sigma} \cong \operatorname{Aut}_{\Gamma-G}\left(P_{\rho}\right)=\operatorname{Aut}_{\Gamma-G}\left(\Gamma \times{ }_{\rho} G\right),
$$

where the structure of the group in the right hand side is described in (4-2) and (4-5).

Now we go back to (7-5) and examine the space $\operatorname{Map}_{G}(P, M)_{\text {l.c. }}$ of locally constant $G$-equivariant maps from a $G$-bundle $P$ over $\Sigma^{\prime}$ into $M$.

Lemma 7-5 Let $\pi^{\prime}: P \rightarrow \Sigma^{\prime} / \Sigma$ be a $G$-bundle over a covering space. Then for any $G$-manifold $M$, restriction of any locally constant $G$-map $\alpha: P \rightarrow M$ to the fibre $\alpha_{x_{0}}: P_{x_{0}} \rightarrow M$ over $x_{0} \in \Sigma$ is a $\Gamma$-invariant $G$-map. This correspondence gives rise to the following bijective correspondences:

$$
\operatorname{Map}_{G}(P, M)_{\text {l.c. }} \underset{\text { onto }}{\stackrel{1: 1}{\longrightarrow}} \operatorname{Map}_{G}\left(P_{x_{0}}, M\right)^{\Gamma} \underset{\text { onto }}{\stackrel{1: 1}{\longrightarrow}} \mathcal{S}\left[P_{x_{0}} \times_{G} M\right]^{\Gamma},
$$

where $\Gamma$ acts on $M$ trivially and, $\mathcal{S}\left[P_{x_{0}} \times_{G} M\right]$ denotes the set of sections of the fibre bundle $P_{x_{0}} \times_{G} M \rightarrow \Sigma_{x_{0}}^{\prime}$ with fibre $M$.

Proof First, we show that the correspondence is well defined. Let $\alpha: P \rightarrow M$ be a locally constant $G$-equivariant map. Thus, $\alpha$ is constant on each connected component of $P$. Since the connected component of $P$ is in bijective correspondence with $\Gamma$-orbits in the fibre $P_{x_{0}}$ over $x_{0} \in \Sigma$ (the $\Gamma$-orbit corresponding to a connected component of $P$ is obtained by intersecting the component with the fibre $\left.P_{x_{0}}\right)$, the restriction $\alpha_{x_{0}}: P_{x_{0}} \rightarrow M$ is constant on each $\Gamma$-orbit. Hence $\alpha_{x_{0}}$ is $\Gamma$-invariant. This shows that the correspondence in $(7-18)$ is well defined.

The reverse correspondence is given as follows. For any $\Gamma$-invariant $G$-map $\phi: P_{x_{0}} \rightarrow M$, first we note that $\phi$ is constant on each $\Gamma$-orbit in $P_{x_{0}}$. Then, we can define a locally constant $G$-map $\alpha_{\phi}: P \rightarrow M$ by letting the value of $\alpha_{\phi}$ on a connected component $C$ of $P$ to be $\phi\left(C \cap P_{x_{0}}\right)$. We can easily check that the above two correspondences are inverse to each other. This completes the proof. 
In the above correspondence, note that the topological condition of local constancy of a $G$-map $\alpha: P \rightarrow M$ translates to an algebraic condition of $\Gamma$ invariance of its restriction $\alpha_{x_{0}}: P_{x_{0}} \rightarrow M$ to a fibre. Note that $\pi^{-1}\left(x_{0}\right)=\Sigma_{x_{0}}$, where $\pi: \Sigma^{\prime} \rightarrow \Sigma$, is a $\Gamma$-set.

(Proof of Theorem 7-1) By (7-5), Proposition 7-3, and Lemma 7-5, we can rewrite quantities appearing in the definition of $\varphi_{\left[\Sigma^{\prime} \Sigma \Sigma\right]}(M ; G)$ in terms of their restrictions to the fibre over $x_{0} \in \Sigma$ :

$$
\begin{aligned}
\varphi_{\left[\Sigma^{\prime} / \Sigma\right]}(M ; G) & =\sum \varphi\left(\operatorname{Map}_{G}(P, M)_{\text {l.c. }} ; \operatorname{Aut}_{G}(P)_{\Sigma^{\prime} / \Sigma}\right) \\
& {\left[P \longrightarrow \Sigma^{\prime} \Sigma\right] } \\
& =\sum \varphi\left(\operatorname{Map}_{G}\left(P_{x_{0}}, M\right)^{\Gamma} ; \operatorname{Aut}_{\Gamma-G}\left(P_{x_{0}}\right)\right), \\
& {\left[P_{x_{0}} \longrightarrow X\right]_{\Gamma-G} }
\end{aligned}
$$

where $\left[P_{x_{0}} \rightarrow X\right]_{\Gamma-G}$ runs over all isomorphism classes of $\Gamma$-equivariant $G$ principal bundles over a $\Gamma$-set $X$. But this is precisely our definition of $\varphi_{[X]}(M ; G)$ given in (6-13).

Next, we prove the multiplicativity of $\varphi_{\left[\Sigma^{\prime} \Sigma\right]}(M ; G)$. Let $P \rightarrow \Sigma_{1}^{\prime} \amalg \Sigma_{2}^{\prime}$ be a $G$-bundle over a disjoint union of two covering spaces of $\Sigma$. Let $P_{1}$ and $P_{2}$ be the restrictions of $P$ to $\Sigma_{1}^{\prime}$ and $\Sigma_{2}^{\prime}$, respectively. Then the mapping space splits into a product $\operatorname{Map}_{G}(P, M)_{\text {l.c. }}=\operatorname{Map}_{G}\left(P_{1}, M\right)_{\text {l.c. }} \times \operatorname{Map}_{G}\left(P_{2}, M\right)_{\text {l.c. }}$. When $\Sigma_{1}^{\prime}$ and $\Sigma_{2}^{\prime}$ do not have common isomorphic component as a covering space over $\Sigma$, the automorphism group also splits: $\operatorname{Aut}_{G}(P)_{\left(\Sigma_{1}^{\prime} \cup \Sigma_{2}^{\prime}\right) / \Sigma}=$ $\operatorname{Aut}_{G}\left(P_{1}\right)_{\Sigma_{1}^{\prime} / \Sigma} \times \operatorname{Aut}_{G}\left(P_{2}\right)_{\Sigma_{2}^{\prime} / \Sigma}$, because there are no nontrivial $G$-bundle maps between a connected component of $P_{1}$ and a connected component of $P_{2}$. Thus,

$$
\begin{gathered}
\varphi_{\left[\left(\Sigma_{1}^{\prime} \cup \Sigma_{2}^{\prime}\right) / \Sigma\right]}(M ; G)=\sum \varphi\left(\operatorname{Map}_{G}\left(P_{1} \amalg P_{2}, M\right)_{\text {l.c. }} ; \operatorname{Aut}_{G}\left(P_{1} \amalg P_{2}\right)_{\left(\Sigma_{1}^{\prime} \cup \Sigma_{2}^{\prime}\right) / \Sigma}\right) \\
=\left[\sum_{\left[P_{1} \amalg P_{2} \longrightarrow \Sigma_{1}^{\prime} \amalg \Sigma_{2}^{\prime}\right]} \varphi\left(\operatorname{Map}_{G}\left(P_{1}, M\right)_{1 . c .} ; \operatorname{Aut}_{G}\left(P_{1}\right)_{\Sigma_{1}^{\prime} / \Sigma}\right)\right]\left[\sum_{\left[P_{2}^{\prime}\right]} \varphi\left(\operatorname{Map}_{G}\left(P_{2}, M\right)_{\text {l.c. }} ; \operatorname{Aut}_{G}\left(P_{2}\right)_{\Sigma_{2}^{\prime} / \Sigma}\right)\right] \\
=\varphi_{\left[\Sigma_{1}^{\prime} / \Sigma\right]}(M ; G) \cdot \varphi_{\left[\Sigma_{2}^{\prime} / \Sigma\right]}(M ; G) .
\end{gathered}
$$

This completes the proof.

Theorem 7-1 allows us to rewrite identities (6-14) and (6-4) in terms of covering spaces over $\Sigma$.

Corollary 7-6 Let $\Sigma$ be a connected manifold and let $M$ be a $G$-manifold for a finite group $G$. Then, $\Sigma$-associated orbifold invariant of the $n$-fold symmetric orbifold $\varphi_{\Gamma}\left(M^{n} ; G \geq \mathfrak{S}_{n}\right)$, where $\Gamma=\pi_{1}(\Sigma)$, can be written in terms of orbifold invariants of $(M ; G)$ associated to up to $n$-fold (not necessarily connected) 
covering spaces $\Sigma^{\prime} \rightarrow \Sigma$ of $\Sigma$. In fact, we have the following identity between two generating functions:

$$
\sum_{n \geq 0} q^{n} \varphi_{\Gamma}\left(M^{n} ; G\left\langle\mathfrak{S}_{n}\right)=\sum_{\left[\Sigma^{\prime} \longrightarrow \Sigma\right]} q^{\left|\Sigma^{\prime} \Sigma\right|} \varphi_{\left[\Sigma^{\prime} \Sigma\right]}(M ; G) .\right.
$$

Here $\varphi_{\Gamma}\left(M^{n} ; G \backslash \mathfrak{S}_{n}\right)$ corresponds to trivial covering $\varphi_{[\Sigma / \Sigma]}\left(M^{n} ; G \backslash \mathfrak{S}_{n}\right)$, and the second summation is over all isomorphism classes of (not necessarily connected) covering spaces $\Sigma^{\prime}$ over $\Sigma$.

For the case of orbifold Euler characteristics, we even have an infinite product expansion:

$$
\sum_{n \geq 0} q^{n} \chi_{\Gamma}\left(M^{n} ; G \nmid \mathfrak{S}_{n}\right)=\sum_{\left[\Sigma^{\prime} \rightarrow \Sigma\right]} q^{\left|\Sigma^{\prime} / \Sigma\right|} \chi_{\left[\Sigma^{\prime} / \Sigma\right]}(M ; G)=\prod_{\left[\Sigma^{\prime \prime} \rightarrow \Sigma\right]_{\text {conn. }}}\left(1-q^{\left|\Sigma^{\prime \prime} / \Sigma\right|}\right)^{-\chi_{\left[\Sigma^{\prime \prime} / \Sigma\right]}(M ; G)},
$$

where the product is over all isomorphism classes of finite connected covering spaces $\Sigma^{\prime \prime}$ over $\Sigma$.

The above identity reveals a close and precise connection between symmetric products and covering spaces in our particular context.

\section{Number of conjugacy classes of subgroups of a given index}

As before, let $u_{r}(\Gamma)$ be the number of conjugacy classes of subgroups of index $r$ in $\Gamma$ and let $j_{r}(\Gamma)$ be the number of index $r$ subgroups of $\Gamma$. In this section, we use our combinatorial formulae obtained as corollaries of our topological formulae to compute $u_{r}(\Gamma)$ for various $\Gamma$. Formulae for $u_{r}(\Gamma)$ are known for the genus $g+1$ orientable surface group $\Gamma_{g+1}$ [12], the free group on $s+1$ generators $F_{s+1}$, and the genus $h+2$ non-orientable surface group $\Lambda_{h+2}$ [13]. However, our method quickly gives the same result in a uniform way. In fact, we prove a formula which applies to any group $\Gamma$ to calculate $u_{r}(\Gamma)$.

First we recall the formula $\left(6-6^{\prime}\right)$ which relates the numbers $u_{r}(\Gamma)$ to the number of conjugacy classes of homomorphisms into symmetric groups:

$$
\sum_{n \geq 0} q^{n}\left|\operatorname{Hom}\left(\Gamma, \mathfrak{S}_{n}\right) / \mathfrak{S}_{n}\right|=\prod_{r \geq 1}\left(1-q^{r}\right)^{-u_{r}(\Gamma)} .
$$

We study the left hand side in detail using our formula (5-5) on the number of homomorphisms into wreath product:

$$
\sum_{n \geq 0} q^{n} \frac{\left|\operatorname{Hom}\left(\Gamma, G 2 \mathfrak{S}_{n}\right)\right|}{|G|^{n} n !}=\exp \left[\sum_{r \geq 1} \frac{q^{r}}{r}\left\{\sum_{\substack{H \\|\Gamma / H|=r}} \frac{|\operatorname{Hom}(H, G)|}{|G|}\right\}\right] .
$$


The main result in this section is the following formula. Let $\mathbb{Z}_{r}=\mathbb{Z} / r \mathbb{Z}$.

Theorem 8-1 (1) For any group $\Gamma$, the generating function of the number of conjugacy classes of homomorphisms into symmetric groups is described in terms of subgroups of $\Gamma$ as follows:

$$
\sum_{n \geq 0} q^{n}\left|\operatorname{Hom}\left(\Gamma, \mathfrak{S}_{n}\right) / \mathfrak{S}_{n}\right|=\exp \left[\sum_{m \geq 0} \frac{q^{m}}{m}\left\{\sum_{r \mid m} \sum_{\substack{H \\|\Gamma / H|=m / r}}\left|\operatorname{Hom}\left(H, \mathbb{Z}_{r}\right)\right|\right\}\right] .
$$

(2) The number $u_{r}(\Gamma)$ of conjugacy classes of index $r$ subgroups of $\Gamma$ satisfies the following recursive relation in terms of subgroups of $\Gamma$ :

$$
j_{m}(\Gamma \times \mathbb{Z})=\sum_{r \mid m} r \cdot u_{r}(\Gamma)=\sum_{r \mid m} \sum_{\substack{H \\|\Gamma / H|=m / r}}\left|\operatorname{Hom}\left(H_{\mathrm{ab}}, \mathbb{Z}_{r}\right)\right|,
$$

where $H_{\mathrm{ab}}$ denotes the abelianization of $H$.

Proof Using the second formula in (2-6), we have

$$
\begin{aligned}
\left|\operatorname{Hom}\left(\Gamma, \mathfrak{S}_{n}\right) / \mathfrak{S}_{n}\right| & =\frac{\left|\operatorname{Hom}\left(\Gamma \times \mathbb{Z}, \mathfrak{S}_{n}\right)\right|}{\left|\mathfrak{S}_{n}\right|}=\sum_{\sigma \in \mathfrak{S}_{n}} \frac{|\operatorname{Hom}(\Gamma, C(\sigma))|}{\left|\mathfrak{S}_{n}\right|} \\
& =\sum_{[\sigma] \in \mathfrak{S}_{n *}} \frac{|\operatorname{Hom}(\Gamma, C(\sigma))|}{|C(\sigma)|}
\end{aligned}
$$

where $\mathfrak{S}_{n *}$ denotes the set of conjugacy classes in $\mathfrak{S}_{n}$. It is well known that the conjugacy class $[\sigma]$ in $\mathfrak{S}_{n}$ depends only on the cycle type of $\sigma$. Suppose the cycle type of $\sigma$ is given by $\prod_{r>1}(r)^{m_{r}}$ with $\sum_{r} r m_{r}=n$, then it is well known that its centralizer is a product of wreath products given by $C(\sigma) \cong$ $\prod_{r \geq 1}\left(\mathbb{Z}_{r} 2 \mathfrak{S}_{m_{r}}\right)$ [11]. Then the above formula becomes

$$
\left|\operatorname{Hom}\left(\Gamma, \mathfrak{S}_{n}\right) / \mathfrak{S}_{n}\right|=\sum_{\substack{m_{r} \geq 0 \\ \sum m_{r}=n}} \prod_{r \geq 1} \frac{\left|\operatorname{Hom}\left(\Gamma, \mathbb{Z}_{r} \imath \mathfrak{S}_{m_{r}}\right)\right|}{\left|\mathbb{Z}_{r} \imath \mathfrak{S}_{m_{r}}\right|}
$$

Now we form a generating function. Using the above formula, we have

$$
\begin{aligned}
\sum_{n \geq 0} q^{n}\left|\operatorname{Hom}\left(\Gamma, \mathfrak{S}_{n}\right) / \mathfrak{S}_{n}\right| & =\sum_{m_{r} \geq 0} \prod_{r \geq 1} q^{r m_{r}} \frac{\left|\operatorname{Hom}\left(\Gamma, \mathbb{Z}_{r} \imath \mathfrak{S}_{m_{r}}\right)\right|}{\left|\mathbb{Z}_{r} \imath \mathfrak{S}_{m_{r}}\right|} \\
& =\prod_{r \geq 1} \sum_{m \geq 0} q^{r m} \frac{\left|\operatorname{Hom}\left(\Gamma, \mathbb{Z}_{r} \imath \mathfrak{S}_{m_{r}}\right)\right|}{\left|\mathbb{Z}_{r} \imath \mathfrak{S}_{m_{r}}\right|}
\end{aligned}
$$

Algebraic \&S Geometric Topology, Volume 3 (2003) 
Next, we use our crucial ingredient, namely the formula (8-2). Continuing our calculation, we get

$$
\begin{aligned}
& =\prod_{r \geq 1} \exp \left[\sum_{d \geq 1} \frac{q^{r d}}{d}\left\{\sum_{\substack{H \\
|\Gamma / H|=d}} \frac{\left|\operatorname{Hom}\left(H, \mathbb{Z}_{r}\right)\right|}{r}\right\}\right]=\exp \left[\sum_{r, d \geq 1} \frac{q^{r d}}{r d}\left\{\sum_{\substack{H \\
|\Gamma / H|=d}}\left|\operatorname{Hom}\left(H, \mathbb{Z}_{r}\right)\right|\right\}\right] \\
& =\exp \left[\sum_{m \geq 1} \frac{q^{m}}{m}\left\{\sum_{r \mid m}\left(\sum_{\substack{H \\
|\Gamma / H|=m / r}}\left|\operatorname{Hom}\left(H, \mathbb{Z}_{r}\right)\right|\right)\right\}\right] .
\end{aligned}
$$

This proves (1). For (2), we simply take logarithm of the right hand side of (8-1), and compare it with (8-3). Here we may replace $\operatorname{Hom}\left(H, \mathbb{Z}_{r}\right)$ by $\operatorname{Hom}\left(H_{\mathrm{ab}}, \mathbb{Z}_{r}\right)$ since $\mathbb{Z}_{r}$ is abelian. The equality $j_{m}(\Gamma \times \mathbb{Z})=\sum_{r \mid m} r \cdot u_{r}(\Gamma)$ is a well known identity. For example, see [15, p.112]. This completes the proof

Note that once we have (8-4), we can write down the formula for $u_{r}(\Gamma)$ using Möbius inversion formula. This formula says that if $g$ is a function defined on $\mathbb{N}$ and $f(m)=\sum_{r \mid m} g(r)$, then $g(m)=\sum_{r \mid m} \mu(r) f(m / r)$, where $\mu(r)$ is the Möbius function given by $\mu(1)=1, \mu(n)=(-1)^{t}$ if $n$ is a product of $t$ distinct primes, and $\mu(n)=0$ if $n$ is divisible by the square of a prime. We leave the formula (8-4) in the above form because it is simpler.

The numbers $u_{r}\left(\Gamma_{g+1}\right)$ and $u_{r}\left(\Lambda_{h+2}\right)$ were calculated in [12], [13] around mid 1980s. Our formula (8-4) immediately proves their results which were expressed using Möbius inversion formula. But they are equivalent to the formulae below.

Corollary 8-2 The numbers $u_{r}(\Gamma)$ for $\Gamma=\Gamma_{g+1}, F_{s+1}, \Lambda_{h+2}$ are given in the following recursive relation:

$$
\begin{aligned}
& \sum_{r \mid m} r \cdot u_{r}\left(\Gamma_{g+1}\right)=\sum_{r \mid m} j \frac{m}{r}\left(\Gamma_{g+1}\right) r^{\left.2 \frac{g m}{r}+1\right)} \\
& \sum_{r \mid m} r \cdot u_{r}\left(F_{s+1}\right)=\sum_{r \mid m} j_{\frac{m}{r}}\left(F_{s+1}\right) r^{\frac{s m}{r}+1}
\end{aligned}
$$

$\sum_{r \mid m} r \cdot u_{r}\left(\Lambda_{h+2}\right)=\sum_{r \mid m}\left[\left\{j \frac{m}{r}\left(\Lambda_{h+2}\right)-j \frac{m}{r}\left(\Lambda_{h+2}\right)^{+}\right\}(2, r) r^{\frac{m h}{r}+1}+j \frac{m}{r}\left(\Lambda_{h+2}\right)^{+} r^{\frac{m h}{r}+2}\right]$,

where $j_{r}\left(\Lambda_{h+2}\right)^{+}$is the number of index $r$ orientable subgroups, and $(2, r)$ denotes the greatest common divisor. 
The proof of this corollary is a straightforward application of (8-4). We simply note that the abelianizations are given by $\left(\Gamma_{g+1}\right)_{\mathrm{ab}} \cong \mathbb{Z}^{2 g+2},\left(F_{s+1}\right)_{\mathrm{ab}} \cong \mathbb{Z}^{s+1}$, and $\left(\Lambda_{h+2}\right)_{\mathrm{ab}} \cong \mathbb{Z}^{h+1} \oplus \mathbb{Z}_{2}$, and that among index $m / r$ subgroups of $\Lambda_{h+2}$, there are $j_{\frac{m}{r}}\left(\Lambda_{h+2}\right)^{+}$subgroups which are isomorphic to $\Gamma_{\frac{m h}{2 r}+1}$, and there are $j \frac{m}{r}\left(\Lambda_{h+2}\right)-j \frac{m}{r}\left(\Lambda_{h+2}\right)^{+}$subgroups isomorphic to $\Lambda_{\frac{m h}{r}+2}$.

\section{References}

[1] P. Bantay, Symmetric products, permutation orbifolds, and discrete torsion, arXiv:hep-th/0004025

[2] T. Bröcker and T. tom Dieck, Representations of Compact Lie Groups, Graduate Texts in Math. 98 Springer-Verlag New York (1985)

[3] K. S. Brown, Cohomology of Groups, Graduate Texts in Math. 87 SpringerVerlag New York (1982)

[4] J. Bryan and J. Fulman, Orbifold Euler characteristics and the number of commuting $m$-tuples in the symmetric groups, Annals of Combinatorics 2 (1998) 1-6

[5] R. Dijkgraaf, G. Moore, E. Verlinde, and H. Verlinde, Elliptic genera of symmetric products and second quantized strings, Comm. Math. Phys. 185 (1997) 197-209

[6] L. Dixon, J. Harvey, C. Vafa and E. Witten, Strings on orbifolds, Nuclear Physics B 261 (1985) 678-686

[7] F. Hirzebruch and H. Höfer, On the Euler number of an orbifold, Math. Annalen 286 (1990) 255-260

[8] M. J. Hopkins, N. J. Kuhn, and D. C. Ravenel, Generalized group characters and complex oriented cohomology theories, J. Amer. Math. Soc. 13 (2000) $553-594$

[9] A. Kerber and B. Wagner, Gleichungen in endlichen Gruppen, Arch. Math. 35 (1980) 252-262

[10] I. G. Macdonald, Poincaré polynomials of symmetric products, Proc. Camb. Phil. Soc. 58 (1962) 123-175

[11] I. G. Macdonald, Symmetric Functions and Hall Polynomials, Oxford University Press (1995) second edition

[12] A. D. Mednykh, Hurwitz problem on the number of nonequivalent coverings of a compact Riemann surface, Siber. Math. J. 23 (1983) 415-420

[13] A. D. Mednykh and G. G. Pozdnyakova, Number of nonequivalent coverings over a nonorientable compact surface, Siber. Math. J. 27 (1986) 99-106

[14] P. Shanahan, Atiyah-Singer Index Theorem, Lecture Notes in Math. 638, Springer-Verlag New York (1978) 
[15] R. Stanley, Enumerative Combinatorics, Cambridge Studies in Advanced Mathematics, 62 Cambridge University Press Cambridge (1999)

[16] M. Suzuki, Group Theory I, Grundlehren der mathematischen Wissenschaften 247 Springer-Verlag New York (1982)

[17] H. Tamanoi, Generalized orbifold Euler characteristic of symmetric products and equivariant Morava K-theory, Algebraic and Geometric Topology 1 (2001) 115-141

[18] W. Wang, Equivariant K-theory, wreath products, and Heisenberg algebra, Duke Math. J. 103 (2000) 1-23

Department of Mathematics, University of California

Santa Cruz, CA 95064, USA

Email: tamanoi@math.ucsc.edu

Received: 11 February 2002 Revised: 31 July 2003 